\title{
EXPERIMENTAL INVESTIGATION OF \\ VORTEX RINGS AND HELICOPTER ROTOR WAKES \\ USING A LASER DOPPLER VELOCIMETER
}

by

JOHN P. SULLIVAN

S.B. University of Rochester

1967

M.S. Massachusetts Institute of Technology

1969

SUBMITTED IN PARTIAL FULFILLMENT

OF. THE REQUIREMENTS FOR THE

DEGREE OF DOCTOR OF

SCIENCE

at the

MASSACHUSETTS INSTITUTE OF

TECHNOLOGY

June 1973

Signature of Author Signature redacted

Department of Aeronautics and

Astronautics, February 8, 1973

Certified by Signature redacted
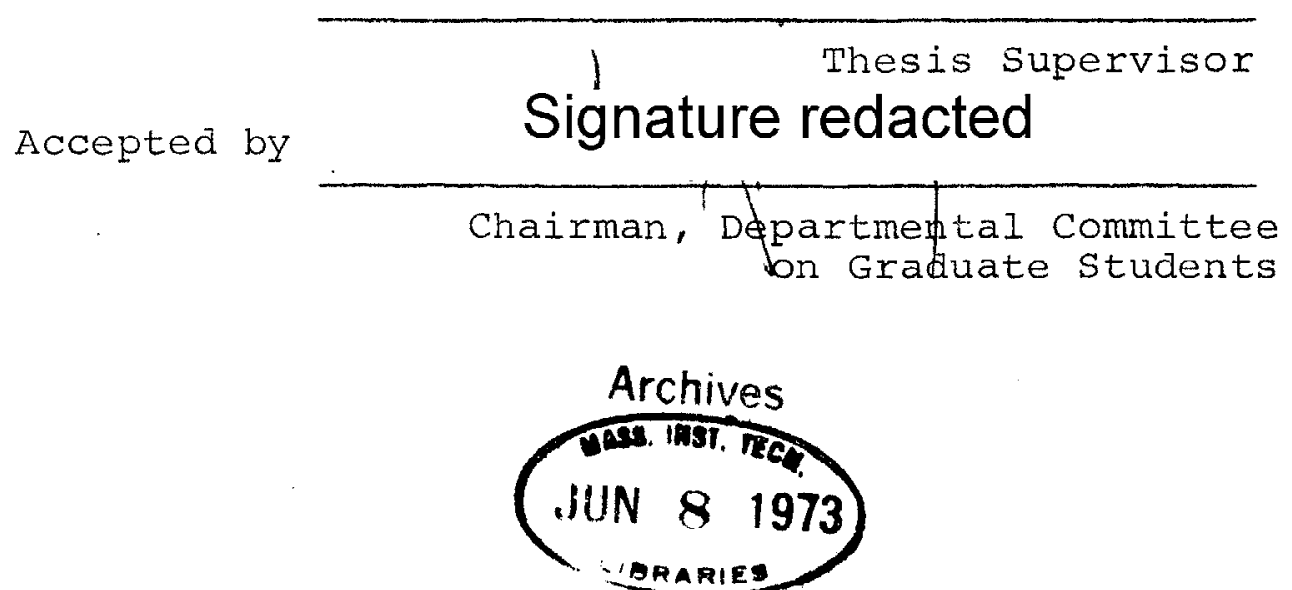
AN EXPERIMENTAL INVESTIGATION OF VORTEX RINGS AND

HELICOPTER ROTOR WAKES USING A IASER DOPPLER VELOCIMETER

by

JOHN P. SULLIVAN

Submitted to the Department of Aeronautics and Astronautics on February 8, 1973 in partial fulfillment of the requirements for the degree of Doctor of science.

\section{ABSTRACT}

This thesis describes a laser Doppler velocimeter (LDV) capable of performing measurements in vortex cores, presents detailed measurements of structure and stability of vortex rings and also presents measurements in the wake of a model helicopter rotor.

The use of conventional instrumentation such as hot wire anemoneter for measuring velocity in vortex filament is hampered by probe interference and the difficulty of calibrating the probe in a flow field where the velocity is changing rapidly in magnitude and direction. For these reasons, a laser Doppler velocimeter (LDV) is used throughout these experiments. The LDV measures the velocity of smoke particles by measuring the Doppler shift of laser light scattered from the particles. The IDV system designed and constructed is a dual scatter system capable of determining two components of the velocity vector.

The most unique feature of the IDV system is the electronics system which is capable of handling the large, fast changes in velocity which are encountered in the flow fields of interest. The electronics system which relies on the fact that the flow fiela is periodic requires only a spectrum analyzer for demodulation and a storage oscilloscope for retaining the data. 
A detailed study of the structure and stability of vortex rings using the two-component LDV system has been made. Vortex rings were generated periodically by pulsing air through a sharp edged orifice using a loudspeaker. The circulation and core size are controlled by the amplitude and duration of the electric signal applied to the loudspeaker.

Detailed surveys of two vortex rings were made; a relatively thick core ring with a ratio of core radius to ring radius a/r $=.27$ and a relatively thin core ring with $a / r=.075$. The principal results show that the vorticity is concentrated in a small core region but that the core is not in solid body rotation.

During the course of the investigation, the fact that vortex rings are unstable was rediscovered. Azimuthal perturbations with between 6 and 16 waves spontaneously develop on the perimeter of the ring and continue to grow. For a given vortex core, only one mode is unstable and the smaller the vortex core, the larger the number of waves in the mode. Theoretical predictions of the mode number are not good, but the correct trend is indicated. The amplification rate is accurately predicted probably because all modes have about the same amplification rate.

A single bladed model helicopter rotor has been constructed. The blade is hollow to allow smoke to be deposited at the tip or any desirable point along the span providing flow visualization and scattering particles for the IDV system.

From LDV data the bound circulation and hence the spanwise load distribution was found. Attempts at using the LDV system to measure the structure of the tip vortex were largely unsuccessful due to random fluctuations in the position of the vortex which could not be handled with the present electronics system.

Thesis Supervisor: Morton Finston

Title: Professor of Aeronautics and Astronautics 
TO JEAN AND LINDA 
ACKNOWLEDGEMENTS

The author wishes to express his sincere thanks to Professor Morton Finston for his overall advice and constant encouragement during this work. Because of the nature of this thesis detailed technical advice was required in several fields. This advice was supplied generously and enthusiastically by Professors "ziggy" Ezekiel in the field of optics, Sheila Widnall in the field of fluid mechanics and Rene Miller in the field of helicopter rotors. Also, the many lively discussions with fellow graduate students, particularly Alan Bilanin and Don Bliss, are gratefully acknowledged.

During the course of this experimental work equipment and advice were scrounged from many sources, particularly the Aerophysics Laboratory, the Research Laboratory for Electronics, and the Aeroprojects Laboratory. I would like to thank the personnel of these laboratories for their generosity.

The manuscript was typed by Helen Putnam, to whom I am most grateful.

Finally, I wish to express long overdue thanks to my wife Jean and daughter Linda for their patience and understanding throughout the doctoral program. 
Financial support for this work was provided by the National Science Foundation, the Naval Air Systems Command (N0019-71-C-0220), the Air Force Office of Scientific Research (F44620-69-C-0090) and by the Joint Services Electronics Program (DAAB 07-71-C-0300). 


\section{TABLE OF CONTENTS}

Chapter No.

I

II

III

IV

V

VI

References

Tables

1

2

3

Figures
Page No.

Introduction

16

21

38

44

56

62

67

Model Rotor Parameters (Single Blade) 73
LDV Characteristics 71

$\begin{array}{ll}\text { Data Summary } & 72\end{array}$

74 


\section{LIST OF ILLUSTRATIONS}

Eigure No.

Page No.

la

Reference Beam System

74

$1 \mathrm{~b}$

Dual scatter system

74

2

Doppler Shift vs. Velocity for He-Ne

Laser at Various Scattering Angles

75

$3 a$

$3 \mathrm{~b}$

4

5

6

7

8

9

10

11

12

13

14

15

16
Time Reference Beam System

Time Dual Scatter System

Doppler Signal

Signals from Reference Beam and Dual scatter Systems

Coordinate System for Mie Scattering

Real and Imaginary Parts of $\mathrm{S}$

Scattering Intensity Functions

Coordinate system for scattering of

Incident Wave in $\hat{k}_{i}$ Direction located

at $\vec{r}_{\mathrm{p}}$

Dual scatter system

One Dimensional LDV System

Beam Pattern for Two Dimensional LDV Looking Toward Laser.

Two Dimensional LDV system

Time Diagrams for Electronic System

Demodulation of Test Signal

Linearity and Velocity Check Using

Spinning Disk
76

76

77

78

79

80

81

82

83

84

85

86

87

88

89 
LIST OF ILLUSTRATIONS (continued)

Eigure No.

Page No.

17

Vortex Ring

90

18 Velocity Distribution Through Vortex Ring

91

19 Setup for Measuring Scattering Intensity Distribution

Scheme of Particle Sizing Based on Scattering Measurements Within the Forward Lobe

21 Coordinate System for Vortex Ring Data Analysis

$\mathrm{U}$ Component of Velocity at $r / r_{0}=-.876$ for Ring No. 1

$V$ Component of Velocity at $r / r_{0}=-1.08$ for Ring No. 1

U Component of Velocity Versus Axial Distance at Various Radial Stations of Ring No. 1

$24 \quad V$ Component Versus Axial Distance at Various Radial Stations of Ring No. I

$\mathrm{U} / \mathrm{u}_{\mathrm{o}}$ Versus $\mathrm{r} / \mathrm{r}_{\mathrm{O}}$ along $\mathrm{z} / \mathrm{r}_{\mathrm{O}}=0$

Partial Derivatives Used for Calculating the Vorticity 
LIST OF ILLUSTRATIONS (continued)

Figure No.

Page No.

Non-dimensional $\omega / r$ Versus $\psi$

105

Non-dimensional Spatial Amplification

Rate $\alpha_{x}$ of the Vortex Ring Instability

as a Function of $\tilde{V}$ and Mode Number $n$

106

34

Multiple Flash Side View Photograph of

$\mathrm{n}=7$ mode instability

107

35

Flow Visualization of the Vortex Ring

Instability $\mathrm{n}=6$

108

36

Flow Visualization of the Vortex Ring Instability $\mathrm{n}=7$

Number of Waves in the Unstable Mode as a Function of $\forall$; X Experiment; O Theoretical Prediction at Maximum Amplification

Percent Radial Perturbation for the Vortex Ring of Fig. 36 Measured at Each Strobe Movie Frame (time between frames $14.5 \mathrm{msec}$ ); --Exponential Growth $\alpha_{x}=.69$; Theoretical Prediction $\alpha_{x}=.64$

Cross Section of Blade -- NACA 0012 
LIST OF IILUSTRATIONS (concluded)

Figure No.

Page No.

45

Strobe Photograph with Smoke Released from slit on Top of Blade

Samples of Photographs Used for Determining Vortex Core Position

47

Position of Tip Vortex

Radial and Axial Position of Tip Vortex

Strobe Photograph Showing a Gradual Increase in Core Diameter and Vortex Breakdown 


\section{LIST OF SYMBOLS}

Chapters II and III

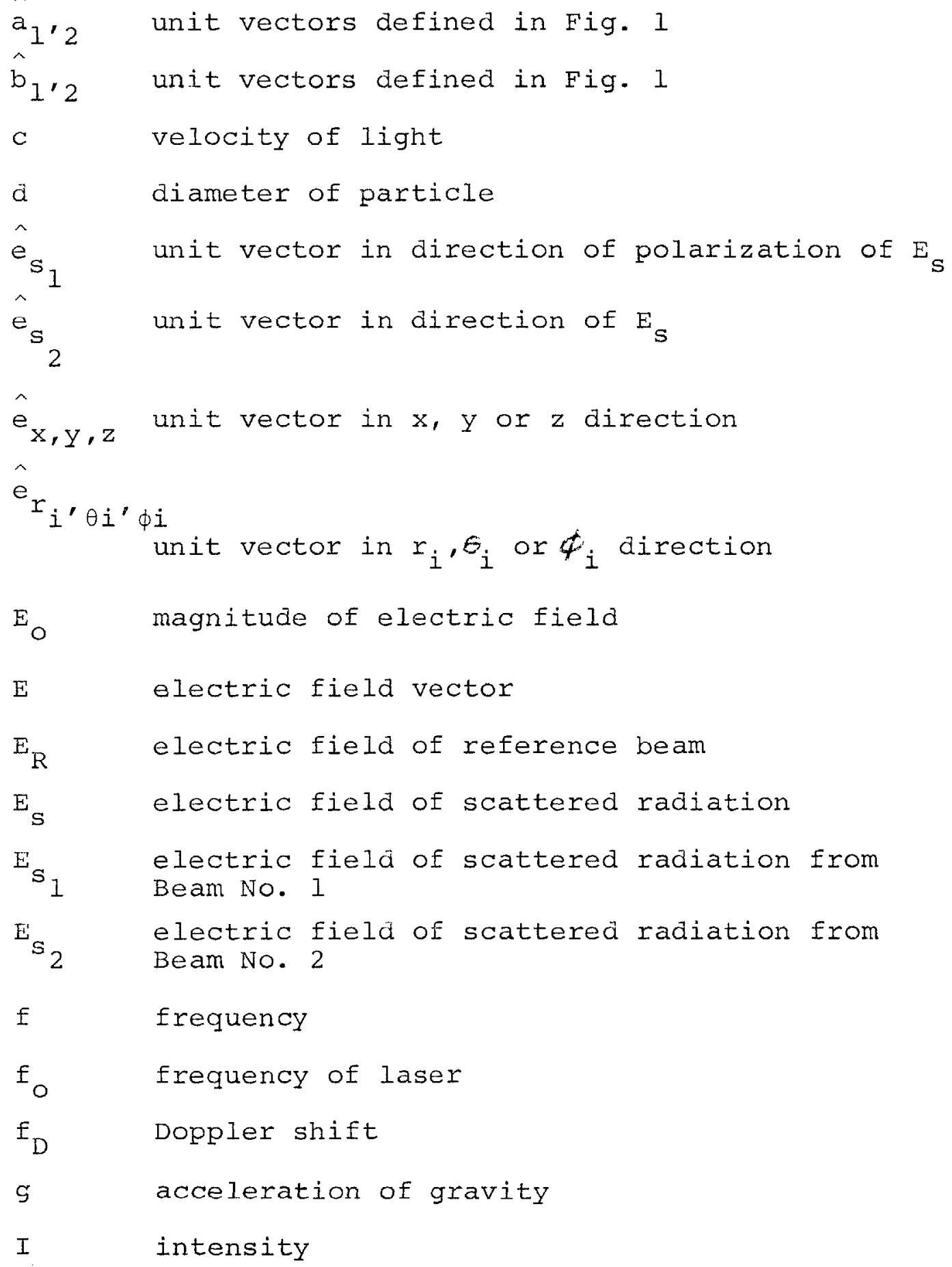


LIST OF SYMBOLS (continued)

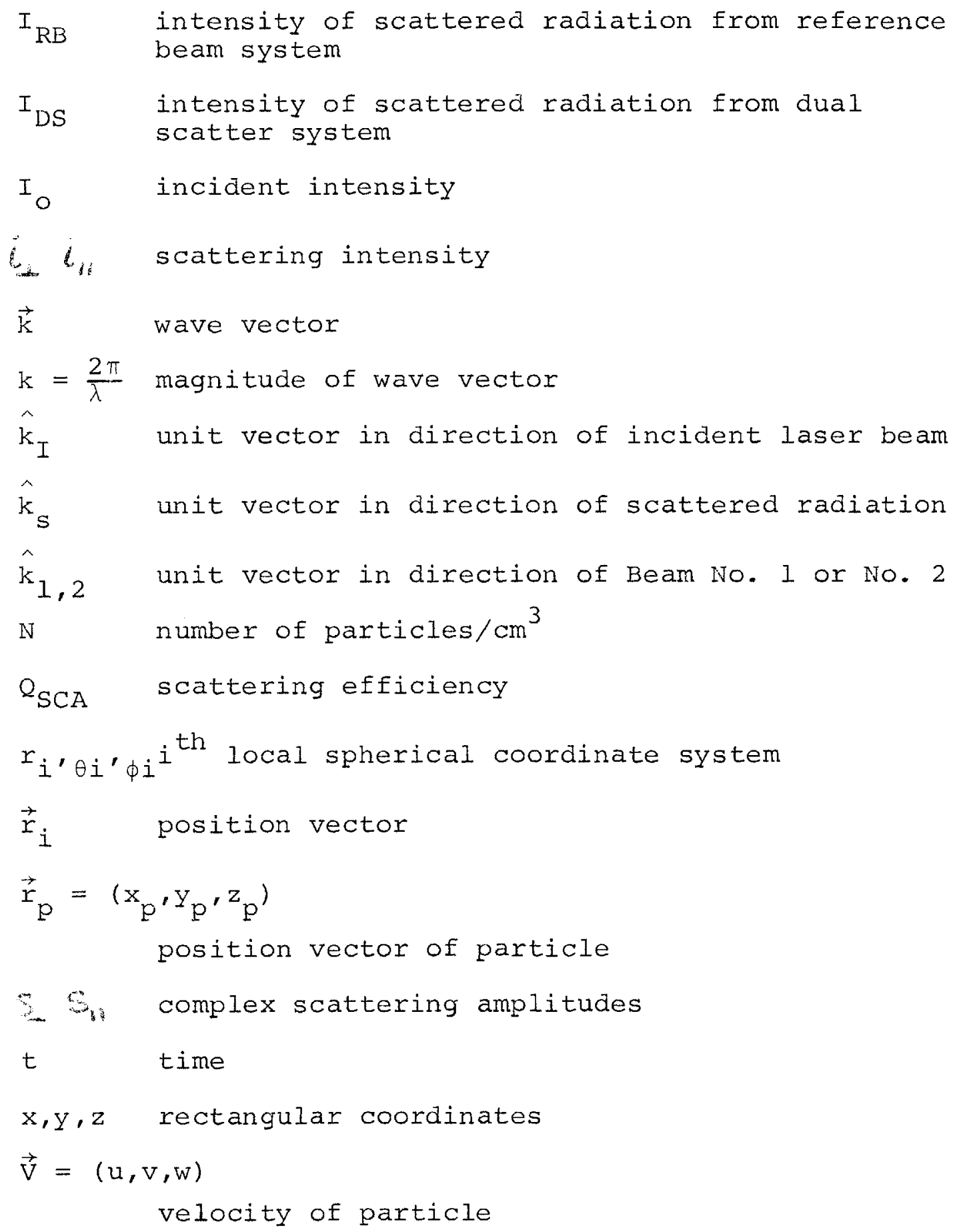




\section{LIST OF SYMBOLS (continued)}

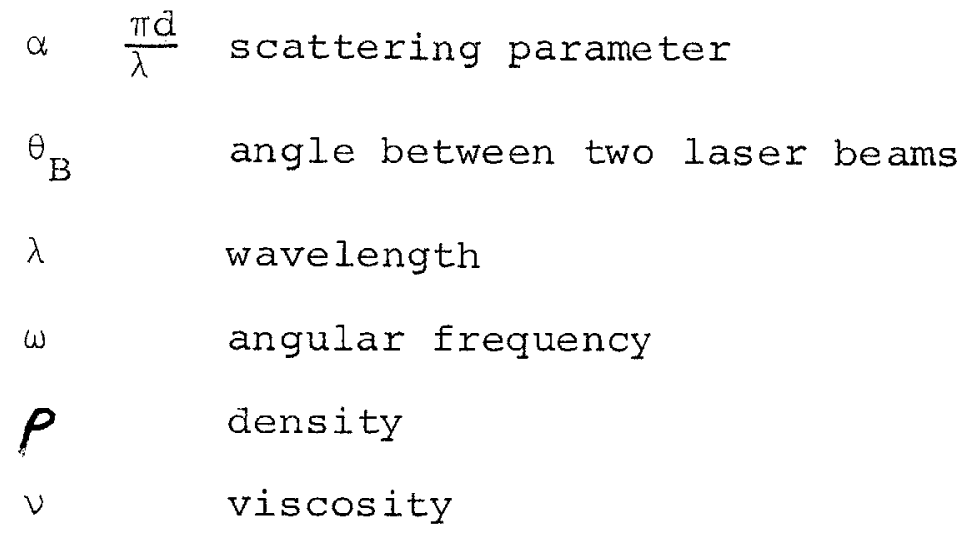

\section{Chapter IV}

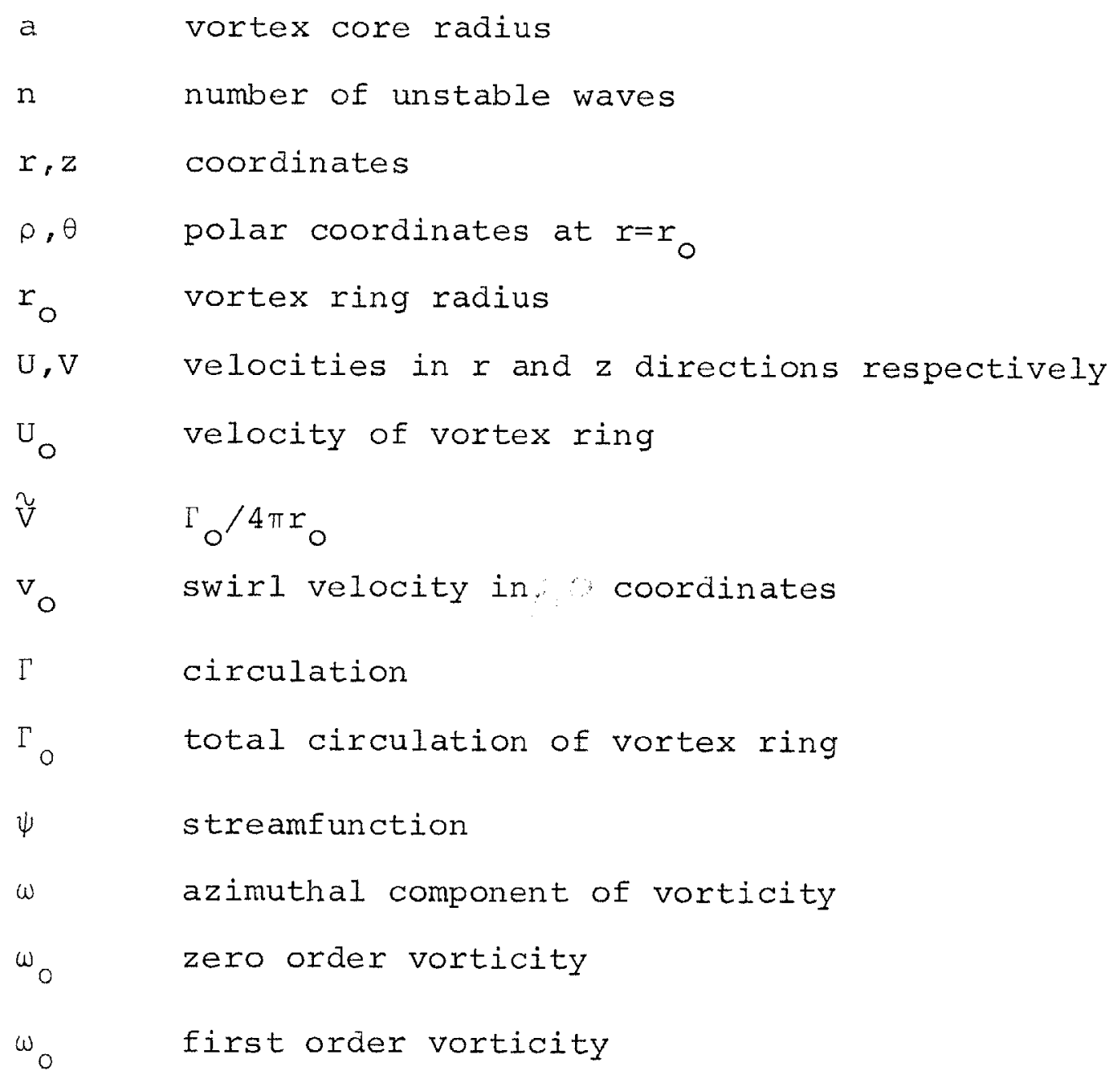


SYMBOLS (continued)

$\begin{array}{ll}\text { Chapter } V & \\ \mathrm{a} & \text { slope of the lift curve } \\ \mathrm{C}_{\mathrm{T}} & \text { thrust coefficient } \\ \mathrm{r}, \theta, \mathrm{z} & \text { coordinates } \\ \mathrm{Ro} & \text { radius of rotor } \\ \mathrm{x} & \text { r/Ro } \\ \Gamma(\mathrm{x}) & \text { bound circulation at } \mathrm{x} \\ \Omega & \text { angular velocity } \\ \sigma & \text { solidity } \\ \theta_{0} & \text { angle of attack }\end{array}$




\section{CHAP TER I}

\section{INTRODUCTION}

\subsection{General Comments}

The current interest in vortex filaments stems from the hazards of the strong vortex wakes of jumbo airliners and the noise and vibration generated by blade vortex interactions on helicopter rotors. Since the induced motion and stability of any vortex filament depends on the detailed structure of the vortex core, it is desirable to make experimental measurements within the core. The purpose of this thesis is to describe a laser Doppler velocimeter (LDV) capable of performing measurements in vortex cores, to present detailed measurements of the structure and stability of vortex rings, and also to present measurements of the flow field of a model helicopter rotor.

\subsection{Literature Review}

\subsubsection{Laser Doppler Velocimeter}

The laser Doppler velocimeter, a device that measures the velocity of particles by measuring the Doppler shift of laser light scattered from the particles was introduced in 1964 by Yeh and Cummins (Ref, 1). They measured the velocity of polystyrene spheres in a water pipe flow. Since then, over 200 papers have been published on the theory, design and application of LDV systems. Two bibliographies of LDV literature are available (Refs. 2 and 3 ), so a detailed review will not be 
presented here. The introduction of the cross-beam reference-beam system by Goldstein and Kreid (Ref. 4) in 1967 and the dual-scatter system by Rudd (Ref. 5) in 1969 have brought the LDV into the realm of a practical fluid-measuring device. These two systems will be discussed in Chapter II where the relevant equations will be derived and appropriate references cited.

1.2.2. Vortex Rings

The study of vortex rings has been pursued for well over a century. The first scientific study was published in 1858 . W. B. Rogers (Ref. 6) observed "rotating" rings in water and air and described the general features: the generation of a ring by an impulse applied across an orifice, the roll-up of the spiral sheets, the formation of a translating ring, and the breakup of rings formed from droplets of a heavier fluid. In $1867 \mathrm{~W}$. Thomson (Lord Kelvin) (Ref. 7) in a note to Tait's translation of Helmholtz's paper on vortex motion, gave, without proof, the correct formulae for the induced velocity of a ring. He then went on to propose (Ref. 8) his theory of vortex atoms based on his observations of the indestructibility of vortex rings. Reynoläs (Ref. 9) in 1876 experimentally observed vortex rings and by comparing the energy and momentum of the ring with that of a circular disk he derived formulae for the ring and core radius relative to the disk radius. In his monograph (Ref. 10), J. J. Thompson derives formulae for the induced motion and modes of oscillation of rings which are valid only to $0\left(l_{n} a / r_{0}\right)$. These results showed the rings to be stable. As will be discussed later, when the theory is carried to $O(1)$ the vortex ring in an ideal 
fluid is in fact unstable. Hicks (Ref. 11), Dyson (Ref. 12) and Pocklington (Ref. 13) carried the theoretical studies of the induced velocity to higher order for the cases of solid body rotation cores and hollow cores. In $1894 \mathrm{Hill}$ (Ref. 14) derived a closed form solution for a sphere of vorticity whose strength varies linearly with distance from the axis of symmetry. Northrop (Ref. 15), Sadron (Ref. 16), and Banerji and Barave (Ref. 17) present numerous photographs and curves of distance traveled versus time, for rings generated by different impulses. In a rather remarkable paper, Krutzsch (Ref. 18) experimentally observed vortex ring instability. His results showed that a sinusoidal perturbation (with 5 to 12 waves around the perimeter) spontaneously appears on the filament and grows in amplitude. Krutzsch also measured velocity distribution in the rings using particle tracer techniques. Although Turner's (Ref. 19) main concern was buoyant vortex rings, the entrainment ideas he presents are also relevant to non-buoyant rings. O'Brien (20) has derived some solutions analogous to Hill's spherical vortex for steady spheroidal vortices. Magarvey and Maclatchy (Ref. 2I) have some nice photographs and zaroodny (Ref. 22) gives a review of the state of vortex rings up to 1966. Using a method of matched asymptotic expansions, Tung and Ting (Ref. 23) attempted to obtain the induced velocity of a vortex ring with a viscously decaying core but these results were later shown to be in error. Widnall, Bliss, and Zalay (Ref. 24), Fraenkel (Ref. 25), and Saffman (Ref. 26) have all obtained formulae for the induced motion of small core rings with arbitrary vorticity distributions. 
Johnson (Ref. 27) presents a summary of the aistance vs. time data and also attempted to measure the structure of the ring using a hot-wire anemometer but due to interference and calibration problems was unsuccessful. Recently two papers, cited in Refs. 28 and 29, have presented experimental flow visualization results for rings generated by distorted holes which show various instabilities. Maxworthy (Ref. 30) also observed some instabilities in his study of the structure of rings. However, his attempts to measure local velocities in the ring, using hydrogen bubble techniques, were unsuccessful. Finally, Fraenkel (Ref. 31) presents some examples related to his earlier paper, including a higher order solution for a ring with a solid body rotation core.

These studies, motivated by the fundamental interest in the motion of a curved vortex, have included numerous flow visualization experiments capable of measuring the ring speed and ring size. There has been no detailed study of the structure of the ring. In fact, due to the sensitivity of the ring to probe interference, even the total circulation of the ring has not been measured. In the present study, the circulation, stream lines, and vorticity distribution are found throughout the ring.

1.2.3. Helicopter Rotors

In recent years, complex free wake models for helicopter rotor wakes in both hover and forward flight have been developed by Scully (Ref. 32) and Langrebe (Ref. 33). Due to a lack of experimental information, various assumptions about the structure of the tip vortex and its subsequent interaction with the 
following blade were made. Ideally, a set of experiments that measures the tip vortex structure and location, and detailed blade loading, is needed. The structure of the tip vortex for a fixed wing in a wind tunnel has been measured by Chigier and Corsiglia (Ref. 34) and Rorke, Moffitt and Ward (Ref. 35). Their results are in qualitative agreement but differ in quantitative detail indicating the need for more extensive investigation. Cook (Ref. 36) has recently made some preliminary measurements in the tip vortex of a full-scale rotor on a test stand. His results indicate a significantly smaller core relative to the blade chord than that measured by Refs. 34 and 35 . 
CHAPTER II

THE LASER DOPPLER VELOCIMETER

2.1. Introduction

The principle of operation of two types of laser Doppler velocimeters will be considered in this chapter. A detailed description of the scattering process, operation, construction, and associated electronics for one of these--the dual scatter system-- will also be given.

2.2. Basic Principles of Laser Doppler Velocimetry

Since the introduction of the laser Doppler velocimeter (IDV) in 1964 by Yeh and Cummings (Ref 1) numerous systems have been reported. Two of these, the cross beam-reference beam system (Fig. la) of ref. 2, and the dual scatter system (Fig. Ib) of refs 3 and 37 will be considered. These two were chosen because they are self aligning; that is, once the beams are made to intersect at the probing point and pass through the aperture in front of the photomultiplier tube, the system is aligned. This eliminates the tedious alignment and vibration problems of other systems.

Laser light that is scattered from particles (smoke, dirt, polystyrene spheres) moving at the local fluid velocity undergoes a Doppler shift given by

$$
f=f_{0}+\frac{\vec{V} \cdot\left(\hat{k}_{s}-\hat{k}_{I}\right)}{R}+O\left(\frac{|\vec{V}|}{C}\right)
$$


where

$$
\begin{aligned}
\hat{f}_{O^{\prime}} \lambda, \hat{k}_{I}= & \begin{array}{l}
\text { frequency, wavelength and unit vector in the } \\
\text { propagation direction of the incident laser } \\
\text { beam }
\end{array} \\
= & \text { velocity of particle } \\
\overrightarrow{\mathrm{V}} \quad & \text { unit vector in the direction that the scattered } \\
\hat{\mathrm{k}}_{\mathrm{S}} & \text { light is detected } \\
\mathrm{C} \quad & \text { velocity of light }
\end{aligned}
$$

The frequency of the scattered radiation $f$, which is of the order of light frequencies $\left(\sim 5 \times 10^{14} \mathrm{cy} / \mathrm{sec}\right)$ can be detected either directly using a Fabry-Perot interferometer or can be heterodyned with some light wave at a known frequency to obtain a signal whose frequency is equal to the shift of the laser light frequency. The present resolution of Fabry-Perot interferometers is only $\sim 2 \mathrm{MHz}$ which implies a velocity resolution of $\sim 10 \mathrm{ft} / \mathrm{sec}$. For the present study this is inadequate so a heterodyning system is used.

In the two types of heterodyne systems studied the crossbeam reference beam system (Fig. la) heterodynes the scattered radiation with radiation at the laser frequency $f_{0}$ and the dual scatter system (Fig. Ib) heterodynes the scattered radiation of two different incident beams. In both cases the difference frequency is calculated, using Eq. 1 and the geometry of Fig. 1 , to be

$$
f_{0}=\frac{2 u \sin \frac{\theta}{2}}{\lambda}
$$


where $U$ is the velocity component in a direction perpendicular to the bisector of the angle between two beams. Fig. 2 shows $\mathrm{f}_{\mathrm{D}}$ versus $U$ for various values of $\theta$ with $\lambda=6328 \AA$, the wavelength of a He Ne laser. It should be noted that there is a $180^{\circ}$ amgiguity in determining direction of $U$. The plus or minus direction of $U$ must be determined by prior knowledge or flow visualization.

The output signal of the photomultiplier (P.M.) tube is proportional to the intensity which for the reference beam system is

$$
\begin{aligned}
& I_{R B}=\left(\vec{E}_{R}+\vec{E}_{S}\right) \cdot\left(\vec{E}_{R}+\vec{E}_{S}\right)^{*} \\
& I_{R B}=\left|\vec{E}_{R}\right|^{2}+\left|\vec{E}_{S}(t)\right|^{2}+2 \vec{E}_{R} \cdot \vec{E}_{S}(t) \cos \left(2 \pi f_{D} t\right) \\
& \vec{E}_{R}=\text { electric field of reference beam } \\
& \vec{E}_{S}=\text { scatterea electric field }
\end{aligned}
$$

when a single particle traverses the measuring volume. This produces an output like that shown in Fig. 3a. The d.c. level is essentially constant since $E_{S}(t)<<E_{C}$. The a.c. component is modulated by the term, $E_{R_{S}} E_{s}(t)$ which is generally a gaussian.

In the dual scatter system the light scattered from one beam is heterodyned with light scattered from the other beam resulting in

$$
\begin{aligned}
& I_{0 s}=\left|\vec{E}_{s_{1}}(t)\right|^{2}+\left|\vec{E}_{s_{2}}(t)\right|^{2}+2 \vec{E}_{s_{1}}(t) \cdot \vec{E}_{s_{2}}(t) \cos \left(2 \pi f_{0} t\right) \\
& \vec{E}_{s_{1}}=\text { electric field scattered by beam one } \\
& \vec{E}_{s_{2}}=\text { electric field scattered by beam two }
\end{aligned}
$$

The results for $E_{S_{1}}(t)=E_{S_{2}}(t)$ are shown in Fig. 3b. The d.c. component is now replaced by a low frequency component. The actual signal when a .0002 wire traverses the measuring volume is shown in the oscilloscope trace of Fig. 4a. When the wire doesn't pass 
through the center of the measuring volume a signal (Fig. 4b) containing a large amount of the low frequency component and small amount of the Doppler signal component is obtained.

In the present investigation more than one scatterer will be in the measuring volume at one time resulting in a signal which is the sum of the signals from individual particles. A typical "continuous" signal for a dual scatter system is shown in Fig. 4c. This signal was obtained from a rotating ground glass disk. It shows the general features of dual scattering; namely, a low fre-quency component and low frequency modulation of the Doppler signal. The low frequency modulation is a result of random constructive and destructive interference among the waves scattered by many particles, causing the signal to go randomly to zero during severe destructive interference. This phenomenon, called "drop-out", and the low frequency component, are the major problems in the electronic processing of the signal.

A comparison of the frequency spectrums of the two systems is shown in Fig. 5 for scattering from a ground glass disk and a turbulent jet. Although the magnitudes of the signals are about equal, the low frequency components in the dual scatter system are greater.

The object of this investigation is to determine which of the two systems will give a better signal-to-noise ratio. The reference beam system has the advantage that the signal can be increased by increasing the strength of the reference beam. However, the solid angle through which scattered light may be collected is limited since the Doppler shift $\frac{\vec{V}}{\lambda} \cdot\left(\hat{k}_{s}-\hat{k}_{I}\right)$ 
is a function of viewing direction $\hat{k}_{S}$ so that at large solid angles the Doppler frequency would be unacceptably broadened. on the other hand, the frequency $\frac{\vec{V}}{\lambda} \cdot\left(\hat{k}_{I_{1}}-\hat{k}_{I_{2}}\right)$ of a dual scatter system depends only on the incident beam directions $\hat{\mathrm{k}}_{\mathrm{I}_{1}}, \hat{\mathrm{k}}_{\mathrm{I}_{2}}$ so that the solid angle for viewing scattered radiation is unlimited. However, the strength of the signal from a dual scatter system is determined entirely by the scattering process. Also there will be more low frequency components in the aual scatter system.

This initial investigation did not indicate that one system was clearly superior but that both performed adequately. At this point it was felt that further comparison would not be fruitful, and the dual scatter system was chosen for further study .

2.3. Scattering By a Spherical Particle

The far field solution for the electric field of an incident plane wave traveling in the $z$-direction and linearly polarized in the $\mathrm{x}$-direction

$$
\vec{E}=\hat{e}_{x} E_{0} e^{i(\omega t-k z)}=\text { incident electric field }
$$

scattered by a dielectric sphere, of diameter $d$, located at the origin of the coordinate system (Fig. 6) is given by the Mie solution (Ref. 38).

$$
\begin{array}{r}
\vec{E}_{s}=\frac{i E_{0} e^{i(\omega t-k r)}}{k r}\left[-\sin \phi S_{1}(\theta, \alpha, n) \hat{e}\right. \\
+\cos \phi S_{\|}(\theta, \alpha, n) \hat{\hat{e}} \\
\left.+\hat{e}_{\theta}\right]
\end{array}
$$


26

The complex scattering amplitude $S_{\perp}$ is the scattered component whose electric vector is perpendicular to the plane of observatron (the plane determined by the direction of observation and the direction of propagation of the incident beam) and $S_{11}$ the component parallel to the plane of observation. The amplitudes $S_{1}$ and $S_{11}$ are functions of the azimuthal angle $\theta$, the size parameter $\alpha$

$$
\alpha=\frac{k d}{2}=\frac{\pi d}{\lambda}
$$

and the index of refraction of the sphere, $n$. The solution $\vec{E}_{S}$ is a spherical wave whose polarization depends on the relative amplitudes and phases of the $\hat{e}_{\phi}$ and $\hat{e}_{\theta}$ components.

The intensity of the scattered radiation is

$$
\begin{gathered}
I=\vec{E}_{s} \cdot \vec{E}_{S}^{*} \\
I=\frac{\lambda^{2} I_{0}}{4 \pi^{2} r^{2}}\left[i_{\perp} \sin ^{2} \phi+i_{11} \cos ^{2} \phi\right]
\end{gathered}
$$

where $\quad i_{1}=\left|S_{1}\right|^{2} \quad i_{11}=\left|S_{11}\right|^{2}$

The real and imaginary parts of $S_{\perp}$ and $S_{\| 1}$ are shown in Fig. 7 and $i_{\perp}$ and $i_{11}$ are shown in Fig. 8. Note that for small $\theta$ the values of $i_{\perp}$ and $i_{\|}$are approximately equal and also that there is no phase shift between them (ie. $\frac{\operatorname{Im}\left(S_{\perp}\right)}{\operatorname{RE}\left(S_{\perp}\right)} \simeq \frac{\operatorname{Im}\left(S_{11}\right)}{\operatorname{RE}\left(S_{11}\right)}$ ). This implies that the scattered radiation is linearly polarized.

$$
\text { For small values of } \theta \text { the complex amplitudes } S_{\perp} \text { and } S_{\|}
$$

are equal so

$$
\vec{E}_{S}=\frac{i E_{0} e^{i(\omega t-k r)}}{k r} S_{\perp}\left[-\sin \phi \hat{e}_{\phi}+\cos \phi \hat{e}_{\phi}\right]
$$


If a lens of focal length $f$ is aligned on the z-axis the scattered radiation is transformed to $\Rightarrow$ plane wave

$$
\vec{E}_{S}=\frac{i E_{0} e^{i(\omega t-k z)}}{k f} S_{\perp} \hat{e}_{x}
$$

The polarization of this wave is the same as the incident polarization, a fact that will be used later for separating the components of the velocity vector. Note that this is only valid for small $\theta$, and that the polarization for large $\theta$ will not in general be the same as the incident wave.

In the actual LDV system, the laser beam is focused by a lens of focal length $f$. The electric field in the focal region is given by the plane wave (Ref 46)

$$
\vec{E}=\hat{e}_{x} e^{i(\omega t-k z)}\left(I_{0}\right)^{1 / 2} e^{-\frac{1}{b_{0}^{2}}\left(x^{2}+y^{2}\right)}
$$

$$
b_{0}=\frac{2 F \lambda}{\pi} \text { radius of focused spot to } \frac{1}{e^{2}} \text { intensity points }
$$

where $F=\frac{f}{2 b}=\frac{\text { Focal length }}{\text { Diameter of unfocused laser beam(1/e intensity })}$ and $I_{0}$ is the total integrated intensity. The power of the laser is relatea to $I_{0}$ by

$$
I_{0}=\frac{2 P_{L}}{\pi b^{2}}
$$

which was obtained by integrating $\vec{E} \cdot \vec{E}^{*}$ over the $x-y$ plane.

Although the magnitude of the electric field varies over the particle, this effect will be small since the laser spot size will be $\sim 100$ times the diameter of the particle. The electric 
28

field scattered by a particle in the vicinity of a focused laser

will be

$$
\begin{aligned}
\vec{E}_{s}= & \frac{i\left(I_{0}\right)^{1 / 2}}{k r} e^{i(\omega t-k r)} e^{-\frac{1}{b_{0}^{2}}\left(x_{p}^{2}+y_{p}^{2}\right)} \times \\
& {\left[-\sin \phi S_{\perp}(\theta, \alpha, n) \hat{e}_{\phi}+\cos \phi S_{11}(\theta, \alpha, n) \hat{e}_{\theta}\right] }
\end{aligned}
$$

For future use the scattered field from a particle located at an arbitrary point $\vec{r}_{p}$ by a plane wave with vector $\vec{k}_{i}$ (lying in $z, y$ plane) is found to be

$$
\begin{aligned}
\vec{E}_{s}= & \frac{i\left(I_{0}\right)^{1 / 2}}{k\left|r_{i}\right|} e^{i\left[\left(\omega t-k\left|\vec{r}_{i}\right|-\vec{k}_{i} \cdot \vec{r}_{p}\right]\right.} e^{-\frac{1}{b_{0}^{2}}\left(x_{p_{i}}^{2}+\gamma p_{i}^{2}\right)} \\
& {\left[-\sin \phi_{i} S_{\perp}\left(\theta_{i}, \alpha, n\right) \hat{e}_{\phi_{i}}+\cos \phi_{i} S_{11}\left(\theta_{i}, \alpha, n\right) \hat{e}_{\theta_{i}}\right] }
\end{aligned}
$$

where the subscripted coordinates refer to the coordinate system shown in Fig. 9. Since $|\vec{R}|>>\left|\vec{r}_{p}\right|$ the origin of this coordinate system $\left(x_{i}, y_{i}, z_{i}\right)$ can be taken at the origin of $(x, y, z)$ so that $z_{i}$ lies along $\hat{k}_{i}$ and $y_{i}$ is in the $z, y$ plane. Also

$$
k\left|r_{i}\right|=k\left|R-r_{p}\right| \cong k\left(|\vec{R}|-\frac{\vec{R}}{R} \cdot \vec{r}_{p}\right) \cong k R-\vec{k}_{s} \cdot \vec{r}_{p}
$$


29

So the scattered field is approximately

$$
\begin{array}{r}
\vec{E}_{S}=\frac{i\left(I_{0_{i}}\right)^{1 / 2}}{k R} e^{i\left[\left(\omega t-\left(\vec{k}_{i}-\vec{k}_{s}\right) \cdot \vec{r}_{p}\right)-k R\right]} e^{-\frac{1}{b_{i}^{2}}\left(x_{p_{i}}^{2}+y_{p_{i}}^{2}\right)} \\
\quad\left[-\sin \phi_{i} S_{\perp}\left(\theta_{i}, \alpha, n\right) \hat{e}_{\phi_{i}}+\cos \phi_{i} S_{11}\left(\theta_{i}, \alpha, n\right) \hat{e}_{\theta_{i}}\right]
\end{array}
$$

2.4. Detailed Description of Dual Scatter System

In the dual scatter system, two parallel laser beams are focused at a common measuring point as shown in Fig. 10. The radilation scattered by beam No. $I$ is given by Eq. 2 with $i=1$ and that scattered by beam No. 2 is also given by Eq. 2 with $i=2$. The subscripted coordinates $\left(x_{i}, y_{i}, z_{i}\right)$ or $\left(r_{i}, \theta_{i}, \phi_{i}\right)$ refer to coordinates whose $z_{i}$ axis lies along $\vec{k}_{i}$ with both $\vec{k}_{i}$ and $y_{i}$ in the $\mathrm{z}, \mathrm{y}$ plane. Since the scattering is linear the electric field at any distant point is just the sum of the two scattered waves. The intensity of light, I, scattered by the two beams at any point on a sphere of radius $R$ is given by

$$
\begin{gathered}
I=\left(\vec{E}_{s_{1}}+\vec{E}_{s_{2}}\right) \cdot\left(\vec{E}_{s_{1}}+\vec{E}_{S_{2}}\right)^{*} \\
I=\frac{\lambda^{2}}{4 \pi^{2} r^{2}} e^{-\frac{2}{b_{0}^{2}}\left(x_{p}^{2}+y_{p}^{2} \cos ^{2} \frac{\theta_{g}}{2}+z_{p} \sin ^{2} \frac{\theta_{B}}{2}\right)} \times \\
{\left[\sigma_{1}+\sigma_{2}+2 \sigma_{12} \cos \left(\frac{2 \sin \frac{\theta_{\theta}}{2} y_{p}}{2}\right)\right]}
\end{gathered}
$$


30

where

$$
\begin{aligned}
\sigma_{1}= & I_{0_{1}} e^{-\frac{2}{b_{0}^{2}}\left[\frac{y_{p} z_{p}}{2} \sin \theta_{B}\right]} \times \\
& {\left[\sin ^{2} \phi_{1} i_{1}\left(\theta_{1}\right)+\cos ^{2} \phi_{1} i_{11}\left(\theta_{1}\right)\right] } \\
\sigma_{2}= & I_{0_{2}} e^{+\frac{2}{b_{0}^{2}}\left[\frac{y_{p} z_{p}}{2} \sin \theta_{B}\right]} \times \\
& {\left[\sin ^{2} \phi_{2} i_{1}\left(\theta_{2}\right)+\cos ^{2} \phi_{2} i_{11}\left(\theta_{2}\right)\right] } \\
\sigma_{12}= & \left(I_{0_{1}} I_{O_{2}}\right)^{1 / 2}\left[\sin \phi_{1} \sin \phi_{2}\left(i_{1}\left(\theta_{1}\right) i_{1}\left(\theta_{2}\right)\right)^{1 / 2} \hat{e}_{\phi_{1}} \cdot \hat{e}_{\phi_{2}}\right. \\
& +\cos \phi_{1} \cos \phi_{2}\left(i_{11}\left(\theta_{1}\right) i_{11}\left(\theta_{2}\right)\right)^{1 / 2} \hat{e}_{\theta_{1}} \cdot \hat{e}_{\theta_{2}} \\
& +\sin \phi_{1} \cos \phi_{2}\left(i_{1}\left(\theta_{1}\right) i_{11}\left(\theta_{2}\right)\right)^{1 / 2} \hat{e}_{\theta_{1}} \cdot \hat{e}_{\phi_{2}} \\
& \left.+\cos \phi_{1} \sin \phi_{2}\left(i_{1}\left(\theta_{2}\right) i_{11}\left(\theta_{1}\right)\right)^{1 / 2} \hat{e}_{\theta_{2}} \cdot \hat{e}_{\phi_{1}}\right]
\end{aligned}
$$

Several conclusions can be arawn from Eq. (3). Setting $\vec{r}_{p}=\vec{r}_{o_{p}}+\vec{r} t=$ $\left(x_{o p}+u t, y_{o p}+v t, z_{o p}+w t\right)$

(valid to $\frac{|\vec{V}|}{c}$ ) the a.c. component has a frequency

$$
f_{D}=\frac{2 \sin \frac{\theta_{B}}{2}|v|}{\lambda}
$$


i.e., by measuring this frequency and $\theta_{B}$ the component of velocity in the plane of the two beams and in a direction perpendicular to the bisector of two beams can be determined. The exponent indicates that when a particle is on the ellipsoid

$$
\left(\frac{x_{p}}{b_{0}}\right)^{2}+\left(\frac{y_{p}}{b_{0} / \cos \theta_{\theta / 2}}\right)^{2}+\left(\frac{z_{p}}{b_{0} / \sin \frac{\theta_{\theta}}{2}}\right)^{2}=1
$$

the intensity will be $1 / e^{2}$ of the intensity for a particle located at $(0,0,0)$. This ellipsoid is called the probe volume. Although the probe volume is actually determined by the minimum detectable signal the $1 / e^{2}$ points provide a good estimate. The volume of the probe is

$$
V=\frac{8 \pi b_{o}^{3}}{3 \sin \theta_{B}}
$$

For small values of $\theta_{B}$, the probe is approximately a cylinder with radius $b_{0}$ and length $2 b_{0} / \sin \left(\theta_{B} / 2\right)$.

The signal from a single particle given by Eq. 3 is the sum of two gaussian pulses and the Doppler shift term with the resulting signal discussed earlier and shown in Fig. 4a. The length of the Doppler pulse is controlled by a gaussian envelope

$$
e^{-\frac{2}{b_{0}^{2}}\left[\left(x_{0 p}+u t\right)^{2}+\left(y_{0 p}+v t\right)^{2} \cos ^{2} \frac{\theta_{B}}{2}+\left(z_{o p}+w t\right)^{2} \sin ^{2} \frac{\theta_{\theta}}{2}\right]}
$$

The wider this gaussian envelope the less broad will be the signal in the frequency domain. The best case occurs for $\mathrm{V}=\mathrm{W}=0$

$$
\begin{aligned}
x_{O_{f}}=y_{O_{p}}=z_{O_{p}}=0 \text { giving } \text { a width } \\
\qquad \frac{\Delta f}{f}=\frac{1}{2 F} \cot \theta_{B / 2}
\end{aligned}
$$


It should be noted that this is the minimum broadening that can be expected. Any particle with a $V$ and/or $W$ component and/or not passing directly through the center of the scattering volume $\left(x_{o_{p}} \neq 0, y_{o_{p}} \neq 0, z_{o_{p}} \neq 0\right)$ will cause additional broadening.

A useful approximation, and one that is often valid in practice, is to assume $\theta_{B}<1$ (or $\left(\pi-\theta_{B}\right) \ll 1$ ) and the solid angle $(\Omega)$ over which scattered radiation is collected is also small. Then

$$
\begin{aligned}
& \phi_{1} \equiv \phi_{2} \cong \phi \\
& \theta_{1} \equiv \theta_{2} \equiv \theta \\
& i_{\perp}\left(\theta_{1}\right) \equiv i_{\perp}\left(\theta_{2}\right)=i_{\perp}(\theta) \\
& i_{11}\left(\theta_{1}\right) \equiv i_{11}\left(\theta_{2}\right)=i_{11}(\theta)
\end{aligned}
$$

Also letting $I_{O_{i}}=I_{O_{i}}=I_{0}$ which is the optimum case.

$$
\begin{aligned}
I= & 2 I_{0}\left[\sin ^{2} \phi \hat{i}_{\perp}(\theta)+\cos ^{2} \phi i_{11}(\theta)\right]\left\{e^{-\frac{2}{b_{0}^{2}}\left(x_{p}^{2}+y_{p}^{2} \cos ^{2} \frac{\theta_{\theta}}{2}+z_{p}^{2} \sin ^{2} \frac{\theta_{\theta}}{2}\right)}\right. \\
& \left.x\left[\cos H\left(\frac{y_{p} z_{p} \sin \frac{\theta_{p}}{2}}{b_{0}^{2}}\right)+\cos 2 \pi\left(\frac{2 \sin \frac{\theta_{\theta}}{2} y_{p}}{2}\right)\right]\right\}
\end{aligned}
$$

The term in braces is simply the intensity distribution in the probe volume. This intensity distribution oscillates from $4 I_{0}$ to 0 thus giving rise to a set of interference fringes. The fringe model, first suggestea by Rudd, implies that as a particle moves into a bright fringe, it scatters light, then it moves into a dark fringe, then back into a bright fringe 
scattering light, etc. Note that this model is only valid for small forward or backward scattering.

Until this point, the polarization of the incident beams has been in the $x$-direction, i.e., perpendicular to the plane of the two beams. For the small angle scattering case this maximizes the Doppler shift term of the intensity since the fringe contrast will be maximum. To show this consider the a.c. part of the intensity within the probe volume formed by two incident beams with arbitrary linear polarization

$$
\begin{aligned}
& \vec{E}_{I_{1}}=E_{a_{1}} \hat{a}_{1}+E_{b_{1}} \hat{b}_{1} \\
& \vec{E}_{I_{2}}=E_{a_{2}} \hat{a}_{2}+E_{b_{2}} \hat{b}_{2}
\end{aligned}
$$

where the unit vectors $\hat{a}, \hat{b}$ are shown in section $A A$ of $F i g .1$. The a.c. or Doppler shift term is

$$
\vec{E}_{I_{1}} \cdot \vec{E}_{I_{2}}=\left[-E_{a_{1}} E_{a_{2}} \cos \theta_{B}+E_{b_{1}} E_{b_{2}}\right]
$$

For arbitrary polarization this equation shows that the signal decreases as $\theta_{B}$ increases. If incident beams are polarized in the $\hat{\mathrm{b}}_{1}$ and $\hat{\mathrm{b}}_{2}$ directions (i.e. $\mathrm{E}_{\mathrm{a}_{1}}=\mathrm{E}_{\mathrm{a}_{2}}=0$ ) then the maximum signal will be obtained. In cases where the scattering is not limited to small angles the polarization of the scattered waves (i.e. $S_{\perp}$ and $S_{11}$ ) must be considered.

2.5. Two Dimensional LDV System and Associated Electronics The LDV system constructed for extensive measurements is a dual scatter system. A one-dimensional dual scatter system is shown in Fig. 1I. The laser beam is divided by a standard prismtype beamsplitter with a non-absorbing beamsplitting interface. 
This method of dividing the laser beam is preferable to other systems using mirrors and beamsplitters since only one standard optical component is needed and the path lengths (distance from laser to measuring point) of two beams are equal.

A two-dimensional system is constructed by adding a second. beamsplitter (rotated 90 degrees with respect to the first) to form four parallel beams. The pattern of the four beams is shown in Fig. 12. The polarization of the beams is chosen to maximize the signal (Eq. 4) and to eliminate cross talk between the two dimensions. By placing an analyzing polaroid in front of the P.M. tube either of the velocity components can be chosen with no interference from the other component. This is possible because the polarization is changed only slightly by the scattering process. In the present system, both components are processed simultaneously through one P.M. tube using the electronic system described below. A photograph of the two-dimensional LDV system is shown in Fig. 13. The two components could be processed separately by splitting the scattered radiation with a polarizing type beamsplitter and using two P.M. tubes and two sets of electronics.

The electronic system for processing the LDV signal is one suggested by Ref. 39 for use with periodic flow fields. This "sampling" system will be described for use with periodically produced vortex rings in terms of the time diagrams of Fig. 14 and the equipment layout of Fig. 11. The function generator initiates a pulse which is amplified and used to drive a loudspeaker which forms a vortex ring at a sharp edged orifice. A 
synchronous pulse is also taken off of the function generator (Fig. 14a).

The velocity versus time, as the vortex ring goes past the measuring point, is shown in Fig. 14b. This is proportional to the frequency versus time of the output signal of the P.M. tube which is fed into the input of the spectrum analyzer. The synchronous pulse from the function generator initiates a time delay which starts the sawtooth sweep generator (Fig. 14c) for the spectrum analyzer after a time $t_{D}$. The linear sawtooth represents the center frequency of the narrow filter of the spectrum analyzer. When the frequency from the P.M. tube is equal to the instantaneous frequency of the narrow band pass filter of the spectrum analyzer a "pip" will occur on the spectrum analyzer face and at the spectrum analyzer output (Fig. 14d). This condition can be determined by superposing Fig.14c on Fig. 14b. The linear sawtooth is fed to the input of a gate which is only opened when a "pip" occurs on the spectrum analyzer. The output of the gate -- in this case, three spots -- is stored on a storage oscilloscope. The three spots correspond to a zero frequency marker and two points on the velocity versus time curve we wish to determine. By varying the time delay the remaining points on the velocity curve should be stored.

The results of demodulating a test oscillator signal varying from $50 \mathrm{kHz}$ to $950 \mathrm{kHz}$ at a rate of $33 \mathrm{~Hz}$ are shown in Fig. 15.

The entire LDV system was checked by measuring two components of the velocity of a spinning disk as the disk was traversed across the measuring point. The $x$-component of velocity $U=-w y$ 
should be a constant, and the $y$-component $V=\omega x$ should vary Iinearly as the disk moves in the $\mathrm{x}$-direction. The results of this check, shown in Fig. 16, indicate that the linearity of the electronics system is excellent and that the velocity error is within the exror in determining the r.p.m. of the disk. The ambiguity in determining the direction of $U$ and $V$ is demonstrated in Fig. 16. The U-component is always negative and the $\mathrm{V}$-component is negative for negative values of $\mathrm{x}$.

The two-dimensional system has been used to measure the velocity distribution in periodically produced vortex rings (smoke rings). (The results of extensive measurements are presented in Chapter IV.: The rings are similar to a helicopter rotor wake since regions of time varying concentrated vorticity are produced, yet the flow field is simpler since it is axisymmetric. A picture of a vortex ring is shown in Fig. 17 and the corresponding velocity distributions in Fig. 18. The velocity curves are the $U$ and $V$ components of velocity versus time as the vortex ring travels by the fixed measuring point. There is a $180^{\circ}$ ambiguity in the velocity vector (since frequency is always positive). Flow visualization indicates that the U-component is always positive while the $V$-component is positive to the left of the central zero and negative to the right.

\subsection{Summary of the LDV System}

Using the formulae derived above, the characteristics of the LDV system for a half angle of $\theta_{B} / 2=3.90^{\circ}$ are calculated and summarized in Table 1 . For this angle, which is typical of all 
systems used, the volume from which adequate Doppler signal is received is aporoximately a cylinder.0026" in diameter and $.038 "$ long. On the average there are about five $1.0 \mu$ diameter particles in this cy inder at one time. 
CHAPTER III

PARTICLES FOR USE WITH LDV SYSTEM

\subsection{Introduction}

A laser Doppler velocimeter measures the velocity of particles which are generally assumed to have a velocity equal to the local fluid velocity. Although it is theoretically possible to calculate the fluid velocity from the particle velocity, if they are not equal this would require detailed knowledge of instantaneous particle size which is usually not available. Thus it is important, especially for measurements in air where the particle density is several orders of magnitude times the fluid density, to assure that the particles are small enough to follow the flow.

The purpose of this section is to discuss a method of generating suitable particles, and measuring their diameter and number density. Also a procedure for estimating the difference between the fluid and particle velocity will be described.

\subsection{Particle Generation and Measurement}

In the present experiment oil particles were generated by

a Farval vortex mist lubricator*, a device normally usea for lubricating high speed bearings. The oil particles are generated by mechanically breaking up oil drops at a sonic orifice. This

\footnotetext{
*Farval Mist Lubricator Model No. VM3OB. Available through Fluid Power Division of Eaton, Yale, and Towne, Inc. of Cleveland, Ohio.
} 
results, after filtering, in cold oil particles in the micron diameter size range. This method of obtaining particles was found to be extremely convenient, since it is only necessary to turn on the shop air to obtain suitable particles.

The oil used in the lubricator is called "Fog Juice" * a light oil used in theatrics for producing smoke for graveyard scenes, etc. This oil has the advantage that it will evaporate in about a week, so the laboratory and equipment do not become covered with oil film.

The Iubricator manufacturer specifies that the largest particle size is approximately $2 \mathrm{w}$ in diameter but the size is a function of the oil used. In order to determine the size more accurately the diameter was found by measuring the intensity distribution in the forward scattering lobe. This technique, developed by Hodkinson, Ref 40 , uses the fact that the relative angular distribution of scattered intensity within the Mie forward lobe is a function of size only and not refractive index. The scattering function $i_{\perp}(\theta)$ was measured from $5^{\circ}$ to $150^{\circ}$ using the setup shown in Fig. 19. By forming the ratio of $i_{1}$ at various angles the value of $\alpha=\frac{\pi d}{\lambda}$ can be found using Fig. 20, which is taken from Hodkinson.

The scatter in the data shown in Fig. 20 indicates that there is a distribution of particle sizes but the mean size is $\alpha=5.0$ which gives a mean diameter of $d=1.0 \mu$.

* Type 1964 Fog Juice, available from Mole-Richarason Co., Hollywood, Calif. 
The loss in intensity of a beam of light traversing a distance $\ell$ through a dispersion of $N$ particles $/ \mathrm{cm}^{3}$ is

$$
\frac{I}{I_{0}}=e^{-N Q_{s c a} \pi\left(\frac{d}{2}\right)^{2} \ell}
$$

where $Q_{\text {sca }}$ is the scattering efficiency which is equal to the scattering cross-section divided by $\pi(d / 2)^{2}$. By placing the detector of Fig. 19 at $\theta=0$ and measuring the ratio of intensities with and without particles in the cell the number of particles $/ \mathrm{cm}^{3}$ can be determined. The results of this measurement, using a value $\mathrm{Q}_{\mathrm{sca}}=3.0$ from $\operatorname{Ref} 38$ for $\alpha=5.0$ and $\mathrm{n}=1.5$, give $\mathrm{N}=8.5 \times 10^{6}$ particles $/ \mathrm{cm}^{3}$.

3.3. Motion of Spherical Particles in a Flow Field

In order to estimate the difference between fluid and particle velocities the equation of motion of the particle is non-aimensionalized and the appropriate dimensionless parameters deriveä.

The equation of motion of a small spherical particle subject to stokes drag is (Ref 4I)

$$
\begin{aligned}
\frac{\pi}{6} d^{3} \rho_{p} \frac{d \vec{u}_{p}}{d t} & =-3 \pi \nu_{f} \rho_{f} d\left(\vec{u}_{p}-\vec{u}_{f}\right)-\frac{\pi}{6} d^{3} \nabla p \\
& -\frac{1}{2} \times \frac{\pi}{6} d^{3} \rho_{f}\left(\frac{d \vec{u}_{p}}{d t}-\frac{d \vec{u}_{f}}{d t}\right)
\end{aligned}
$$




$$
\begin{aligned}
& +\frac{3}{2} \rho_{f} d^{2} \sqrt{\pi \nu_{f}} \int_{t_{0}}^{t} d t_{1} \frac{\left(\frac{d \vec{u}_{f}}{d t_{1}}-\frac{d \vec{u}_{p}}{d t_{1}}\right)}{\sqrt{t-t_{1}}} \\
& -\frac{\pi}{6} d^{3} g\left(\rho_{p}-\rho_{f}\right)
\end{aligned}
$$

where $\mathrm{U}=$ velocity

$$
\begin{aligned}
& p=\text { density } \\
& \nu=\text { viscosity } \\
& \dot{a}=\text { diameter of particle }
\end{aligned}
$$

Subscripts $p$ and $f$ refer to particle and fluid respectively. The meaning of the various terms is as follows.

The term on the left-hand side is the force required to accelerate the particle. The first term on the right-hand side is the viscous resistance force according to stokes' law. The second term is due to the pressure gradient in the fluid surrounding the particle, caused by acceleration of the fluid. The third term is the force to accelerate the apparent mass of the particle relative to the ambient fluid. The fourth term is the so-called "Basset" term, which takes account of the effect of the deviation in the stokes flow pattern from steady state. The last term is the force due to gravity. 
42

Non-dimensionalizing with respect to a reference fluid velocity

$U_{R E F}$ and length $I_{R E F}$ and using $\nabla f=-\rho_{f} \frac{d u_{f}}{d t}$

the equation of motion becomes

$\operatorname{stk} \frac{d}{d t^{\prime}}\left(\vec{u}_{p}^{\prime}-B \vec{u}_{f}\right)+\left(\vec{u}_{p}-\vec{u}_{f}\right)-C \int_{t_{0}^{\prime}}^{t^{\prime}} \frac{d t_{1}^{\prime}\left(\frac{d u_{f}^{\prime}}{d t_{1}^{\prime}}-\frac{d u_{p}}{d t_{1}^{\prime}}\right)}{\sqrt{t^{\prime}-t_{1}^{\prime}}}$

$$
+G=0
$$

where all quantities are nondimensional

$$
\begin{aligned}
& \text { StR }=\frac{U_{B E F}}{L_{R E F}}\left(1+\frac{\rho_{f}}{\rho_{p}}\right) \tau=\text { stokes number } \\
& \tau=\frac{d^{2}}{18 v_{f}} \frac{\rho_{p}}{\rho_{f}} \\
& B=3\left(\frac{\rho_{f}}{\rho_{p}}\right)\left(\frac{1}{2+\rho_{f} / \rho_{p}}\right) \\
& C=\frac{1}{2} \sqrt{\frac{18}{\pi}} \sqrt{\frac{s t k}{\left(1+\rho_{P} / \rho_{f}\right)}} \\
& G=\frac{\tau\left(1-\rho_{f} / \rho_{p}\right) g}{U_{R E F}}
\end{aligned}
$$


In the present study oil particles are used in air so $\frac{\rho_{f}}{\rho_{p}}=1 \overline{0}^{3}$ implying $\mathrm{B} \ll 1$ and that $\mathrm{C} \ll$ stk so the equation can be written approximately (The gravity force is also neglected)

$$
\text { stk } \frac{d \vec{u}_{p}^{\prime}}{d t^{\prime}}+\left(\vec{u}_{p}^{\prime}-\vec{u}_{f}^{\prime}\right)=0
$$

The role of the time scale $\tau$ can be seen by considering the simple problem of a particle moving in a straight line with a velocity at $t=0$ of $U_{0}$ in a stationary fluid. At any time $t$ later the velocity will be

$$
\frac{u_{p}}{u_{0}}=e^{-t / \tau}
$$

showing that $\tau$ is the characteristic rise time for a particle to reach the local fluid velocity.

For one micron diameter oil particle in air $\tau=4 \mathrm{x} 10^{-6} \mathrm{sec}$ and the stokes number, which is a measure of the difference between fluid and particle velocities, is

$$
\operatorname{Thus}\left|\frac{\mathrm{U}_{\mathrm{p}}-\mathrm{U}_{\mathrm{f}}}{\mathrm{U}_{\mathrm{REF}}}\right| \sim .4 \% \text { for } \mathrm{U}_{\mathrm{REF}}=10 \mathrm{ft} / \mathrm{sec} \text { and } \mathrm{I}_{\mathrm{REF}}=.01 \mathrm{ft} \text {. }
$$


CHAPTER IV

VORTEX RINGS

\subsection{Introduction}

Although the fundamental interest in vortex rings has led to many flow visualization studies, there have been relatively few attempts to measure the detailed structure of the ring. This is due in part to the sensitivity of flows with concentrated vorticity to the insertion of a probe.

Tracer techniques were attempted by Krutzsch (Ref 18) in 1939 using aluminum flakes and Maxworthy (Ref 30) in 1970 using hydrogen bubbles but due to strong axial gradients and associated radial velocities their results were inaccurate. Hot wire measurements (Ref 27) are hampered by probe interference and the difficulty of calibrating the probe in a flow field where velocity is changing rapidly in magnitude and direction. These problems are circumvented in the present experiment by use of a laser Doppler velocimeter (LDV).

Although the formation process cannot be predicted, there are two analytical treatments of steady vortex rings, the small core vortex where the ratio of core size to ring radius is small and Hill's spherical vortex where vorticity is distributed throughout a sphere. Several recent studies (Refs $24 \& 26$ ) of the motion of small core vortex rings give the translational velocity of the 
ring as (from Ref 24)

$$
u_{0}=\Gamma_{0} / 4 \pi r_{0}\left[\ln \frac{8 r_{0}}{a}+A-1 / 2\right]
$$

where $A$ is an integral over the tangential velocity distribution in the core (defined later). For solid body rotation in the core $A=1 / 4$ giving the classical Kelvin results. From the above equation the relevant non-dimensional parameter to characterize the structure of the vortex core is

$$
\tilde{V}=u_{0} / r_{0} / 4 \pi r_{0}
$$

Since the term in braces depends on the detailed distribution of vorticity it is of interest to measure this distribution. Also, models for studying the stability or entrainment will depend on the detailed structure of the ring. In the present study the circulation, streamlines and vorticity distribution are found throughout the ring from measurements of the axial and radial velocities made prior to instability and compared to theoretical predictions.

4.2. Description of the Experiment

Vortex rings are generated by pulsing air through a sharpedged orifice using a loudspeaker. (The circulation and core size are controlled by the amplitude and duration of the electrical signal applied to the loudspeaker.) (see Fig. Il.)

The electronic system for processing the LDV signal is a data sampling system that requires a periodic flow field. To obtain a complete velocity profile, the rings are produced periodically; the distance between rings was approximately 100 ring radii to prevent any interaction between the rings. 
The LDV system measures two components of velocity versus time at a fixed point in space as the vortex ring goes by the measuring point. The velocity distribution is transformed to the steady coordinate system of Fig. 2 I by letting $\mathrm{z}=\mathrm{U}_{\mathrm{O}} \mathrm{t}$ where $\mathrm{U}_{0}$ is the instantaneous velocity of the vortex ring at the measuring point. This quasi-steady approximation assumes that the properties of the vortex ring do not change (due to entrainment of surrounding fluid or wake shedding) significantly in the time it takes the vortex ring to pass the measuring point. This is justified by flow visualization studies of the variation of ring speed $U_{0}$ and size of the ring.

Detailed surveys of a relatively thick core ring (denoted Ring No. 1) and a thin core ring (Ring No. 2) were made. Complete two dimensional data were taken for Ring No. 1 but only the component of velocity in the direction of ring travel was measured for Ring No. 2 .

Two samples of the velocity versus time data from Ring No. 1 are shown in Fig. $22 \mathrm{a}$ and $22 \mathrm{~b}$. There is a $180^{\circ}$ ambiguity in the direction of velocity (since the detected frequency shift is always positive) which is easily resolved by flow visualization indicating that the $U$ component (Fig. 22a) is always positive and the $V$ component (Fig. 22b) is negative to the left of the central zero and positive to the right. Data for Ring No. 2 is similar with velocities being five times greater and the time scale five times faster since the ring speed $U_{0}$ of Ring No. 2 is about five times that of Ring No. 1 . 
Prior to data analysis the Polaroid oscilloscope pictures are photographically enlarged 2.5 times and a smooth curve drawn through the numerous dots. The results of this enlargement are shown in Figs. 23 and 24. These curves represent a portion of the data taken on Ring No. 1. The remaining data on Ring No. 1 were taken in the vicinity of the core with an expanded velocity scale.

4.3. Results and Discussion

The axial component of velocity $U / U_{0}$ versus $r / r_{0}$ along $z / r_{0}=0$ for the two vortex rings and Hill's spherical vortex for comparison is shown in Fig. 25. The value of $r_{0}$ is defined by the zero crossing of this curve and the core radius as one-half the distance between the positive and negative peak velocities. This gives a ratio of core radius to ring radius of $\mathrm{a} / \mathrm{r}_{\mathrm{o}}=0.27$ for Ring No. I and $a / r_{0}=0.075$ for Ring No. 2 , indicating that Ring No. 2 is a relatively thin core ring while Ring No. 1 has a relatively thick core but not nearly as thick as Hill's vortex where $a / r_{0}=1.414$. No data were taken in the core of Ring No. 2 due to the lack of smoke particles in the vicinity of the core. The circulation $\Gamma(r)$ is obtained by integrating the velocity along the curve C (Fig. 21); a rectangle with one side along $r=$ constant and the other sides tending to infinity. Thus

$$
\Gamma=\int_{-\infty}^{\infty}\left(u(z)-u_{0}\right) d z
$$


In order to correct for the finite extent of the data it is assumed that $\left(U(z)-U_{0}\right) \sim 1 / z^{3}$ after the last data point, which gives

$$
\begin{array}{r}
\Gamma=\int_{z_{1}}^{z_{2}}\left(u(z)-u_{0}\right) d z+1 / 2\left[u\left(z_{2}\right) z_{2}\right. \\
\left.-u\left(z_{1}\right) z_{1}\right]
\end{array}
$$

where $z_{2}$ is the upstream cutoff and $z_{1}$ is the downstream cutoff. The circulation was determined by finding the area under curves like Fig. $22 \mathrm{a}$ using a planimeter and adding the small correction from (5) due to the finite extent of the data. This correction was always less than 5\%. The total circulation is the integral along $r=0$. The resulting circulation distribution (Fig. 26) indicates again that Ring No. 2 is the thinner core ring having the circulation more concentrated near $r / r_{0}=1.0$. The circulation calculated in this manner can give some indication of the vorticity distribution by differentiating the curves of Fig. 26. However, in order to obtain the vorticity this derivative would have to be multiplied by an appropriate length corresponding to the distribution of vorticity in the $z$ direction. That is, the slope of the curves of Fig. 26 represents the vorticity weighted by an axial distribution length which is unknown. An alternate procedure will be used to determine the vorticity distribution.

A summary of the data is given in Table 2. A comparison between the actual value of $\widetilde{V}=U_{0} / \Gamma_{0} / 4 \pi r_{0}$ and the value calculated for a small core, solid body rotation ring, In $8 \mathrm{r} / \mathrm{a}-1 / 4$, shows that the latter is 30-40\% higher. This is due to the somewhat arbitrary definition of $a$ and the fact that the core is 
not in solid body rotation which implies $A$ has a value other than $1 / 4$.

Since both components of velocity were measured on Ring No. I the vorticity distribution throughout the vortex ring can be found. In order to minimize differentiation of data the fact that $\omega / r$ is constant on a streamline for a steady axisymmetric flow is utilized. The streamlines are found for the entire flow field and the value of $\omega$ is calculated only along $z / r_{0}=0$ giving a value of $\omega$ on each streamline so $\omega$ is known everywhere.

Integration of velocity along convenient curves gives the stream function $\psi$ so that the streamlines, lines of constant $\psi$ can be plotted. For an axisymmetric flow

$$
d \psi=r u d r-r V d z
$$

Integrating from $\mathrm{z}=+_{\infty}$ along a line $r=$ constant gives

$$
\psi(r=\text { const. } z)-\psi(r=\text { const, } \infty)=r \int_{-\infty}^{z} V\left(z^{\prime}\right) d z^{\prime}
$$

If we take $V \sim 1 / z^{3}$ after some cutoff $z_{1}$ and non-dimensionalizing

$$
\begin{aligned}
\frac{\psi(r, z)+\frac{1}{2}\left(\frac{r}{r_{0}}\right)^{2}}{r_{0}^{2} u_{0}}=\frac{r}{r_{0}}\left[\frac{v\left(z_{1}\right) z_{1}}{2 u_{0} r_{0}}\right. \\
\left.-\int_{z_{1}}^{z_{2}} \frac{v\left(z^{\prime} / r_{0}\right)}{u_{0}} d\left(z^{\prime} / r_{0}\right)\right]
\end{aligned}
$$

The stream function is thus determined by integrating curves like Fig. 22b, with the results shown in Fig. 27. 
As a check the stream function along $\mathrm{z} / \mathrm{r}_{0}=0$ is calculated by integrating the $U$ component of velocity in Fig. 25 using

$$
\frac{\psi\left(r_{0}\right)}{r_{0}^{2} u_{0}}=\int_{0}^{r / r_{0}}\left(\frac{r^{\prime}}{r_{0}}\right) \frac{u\left(r^{\prime} / r_{0}\right)}{u_{0}} d\left(\frac{r^{\prime}}{r_{0}}\right)
$$

The values of $\psi$ along $z / r_{0}=0$ from the two methods are shown in Fig. 28. This critical check of calculating $\psi$ from different data sets indicates that the overall consistency of the data is very good. The actual streamlines are shown superposed on a strobe photograph of Ring No. 1 in Fig. 29.

The vorticity, $\omega$, is calculated along $z=0$ differentiating the data

$$
\frac{\omega(r, 0)}{\left(u_{0} / r_{0}\right)}=\left.\frac{\partial\left(\frac{v}{u_{0}}\right)}{\partial\left(z / r_{0}\right)}\right|_{z=0}-\left.\frac{\partial\left(\frac{u}{u_{0}}\right)}{\partial\left(r / r_{0}\right)}\right|_{z=0}
$$

The first term is the slope of the curves of Fig. $22 \mathrm{~b}$ at $z=0$ and the second term is obtained by differentiating the smoothed curve of Fig. 25. The results (shown in Fig. 30) of differentiating the first term show scatter in the immediate vicinity of $r / r_{0}=1$ caused in part by the directional ambiguity of the LDV system. The $\mathrm{V}$ component of velocity rapidly changes from a large positive value to a large negative value giving data curves (Fig. 22b) in which the determination of the slope at $z=0$ is difficult. The vorticity distribution along $z=0$ (Fig. 31) shows the expected concentration of vorticity in the core. Using the fact that $\omega / r$ is constant on streamlines in conjunction with Fig. 29 and 31, we can find the vorticity at any 
point in the ring. It is estimated that roughly $85 \%$ of the vorticity is within the $\psi / r_{0}^{2} U_{0}=.4$ streamline.

From the circulation distribution (Fig. 26) it is expected that Ring No. 2 would have an even more concentrated vorticity distribution. These results are in contrast to Maxworthy's (Ref 30) claims that the vorticity is more uniformly distributed throughout the vortex ring. It is felt that this discrepancy is mainly aue to the inaccuracy of the hydrogen bubble technique although Maxworthy's rings may be slightly thicker than the ones presented here.

Also shown in Fig. 31 are the vorticity distribution for a small core solid body rotation ring (using the measured value of $\left.a / r_{0}\right)$ and Hill's spherical vortex. This comparison shows that for rings of the same size and velocity of translation neither model properly describes the experimentally produced vortex rings. In a steady axisymmetric flow $\omega / r=F(\psi)$. Most theories assume $F(\psi)$ is a constant since this is the only tractable case, but Fig. 32 shows that this is not the case.

\subsection{Ring Properties from Vorticity Measurements}

From the measured vorticity distribution along $z / r_{0}=0$, we can calculate the circulation and the velocity parameter $\tilde{V}$ by using the theory presented by Bliss (Ref 24). In this work, the solution for the vorticity distribution within a slightly curved vortex filament was obtained by the method of matched asymptotic expansions. This theory shows that the vorticity 
distribution in a slightly curved filament may be written

$$
\omega(p)=\omega_{0}(p)+\frac{a}{r_{0}} \omega_{1}(p) \sin \theta
$$

where $\omega_{0}$ is the vorticity distributed in a straight filament with radial symmetry and $\omega_{1}$ is the small perturbation due to curvature. $\omega_{0}$ is symmetric about $r=r_{0}(\rho=0)$ and $\omega_{1}$ is antisymmetric. We may use these properties to obtain $\omega_{0}$ directly from the measured data since $\omega_{0}$ is then the average value of the measured vorticity for points equally spaced about $r / r_{0}=1$.

If $w_{0}$ is known for a vortex ring, the circulation and ring velocity can be calculated. The functions $\omega_{0}$ and $\omega_{1}$ are shown in Fig. 23 .

$$
\begin{aligned}
& \text { The circulation of the ring in terms of } \omega_{0} \text { is just } \\
& \Gamma_{0}=2 \pi \int_{0}^{\infty}\left(\frac{p}{r_{0}}\right)\left(\frac{\omega_{0}}{u_{0} / r_{0}}\right) \frac{d p}{r_{0}}\left[u_{0} r_{0}\right]
\end{aligned}
$$

Direct numerical integration of $\omega_{0}$ calculated from the measured vorticity $\omega$ gives a value for $\Gamma_{0}$ of $1.17 \mathrm{ft}^{2} / \mathrm{sec}$ in close agreement with the measured value $1.21 \mathrm{ft} / \mathrm{sec}$ (Table 1 ). The non-dimensional ring velocity $\widetilde{V}$ can also be determined from the measured vorticity. The non-dimensional self-induced velocity of a vortex ring with an arbitrary distribution of vorticity is given by

$$
\tilde{v}=\ln \frac{8 r_{0}}{a}+A-1 / 2=\ln 8-1 / 2+\left(A-\ln a / r_{0}\right)
$$


where $A=\lim _{\bar{\rho} \rightarrow \infty} \int_{0}^{\infty} V_{\theta}^{2}(\bar{\rho}) \bar{\rho} d \bar{\rho}-\ln \bar{\rho}$

$\hat{v}_{0}$ is the non-aimensional swirl velocity (in $\rho, \theta$ coordinate system) $\hat{v}_{0}=v_{0} 2 \pi a / I_{0}$ and $\vec{\rho}=\frac{\rho a}{r_{0}}$ where a is some typical dimension in the vortex core. (Actually, the parameter A - In a/ro is independent of the choice of length scale.)

Straightforwara manipulation yields a formula for the group A - In $a / r_{0}$ in terms of the zeroth-order vorticity $\omega_{0}$. The results being

$$
A-\ln a / r_{0}=\frac{-2 \int_{0}^{\infty} \bar{\rho} \omega_{0}(\bar{\rho}) \ln \bar{\rho} \int_{0}^{\bar{\rho}} \bar{\rho}^{\prime} \omega_{0}\left(\bar{\rho}^{\prime}\right) d \bar{\rho} d \bar{\rho}}{\left[\int_{0}^{\infty} \bar{\rho} \omega_{0}(\bar{\rho}) d \bar{\rho}\right]^{2}}
$$

If the vorticity is uniform within a radius $r=a$, this expression gives the familiar result $A=1 / 4$. Direct numerical integration of this formula using the values of $\omega_{0}$ obtained from the measured vorticity gives $\tilde{V}=3.01$ as compared with a measured value of 2.46 , an error of $20 \%$. This is in somewhat better agreement than the value 3.14 obtained by assuming uniform vorticity. Since the ratio of core size to radius is 0.27 perhaps a $20 \%$ discrepancy between experiment and an asymptotic theory which assumes $a / r<1$ is not surprising.

A value of $\mathrm{A}=0.136$ is obtained for the vorticity distribution of the ring for which $a / r_{0}=0.27$.

4.5. Stability

During the study of the structure of vortex rings it was observea that rings were unstable to azimuthal perturbations, a 
fact that had been reported by Krutzsch (Ref 18) in 1939. An analytical study by widnall (Ref 42) concluded that all vortex rings were unstable to these azimuthal perturbations with the number of unstable waves, $n$, being a function of the nondimensional ring velocity $\tilde{V}=U_{0} / \Gamma_{0} / 4 \pi r_{0}$. For a given vortex core only one mode is unstable and the smaller the vortex core (i.e. the higher the value of $\forall$ ) the larger the number of waves in the mode. This result is shown in Fig. 33 from Ref 42 .

A side view multiple-flash strobe photograph of an $n=7$ mode instability, Fig. 34, shows that the ring appears to be completely destroyed. For a higher mode case, another ring traveling at a slower velocity is formed after the initial instability.

Front view photographs of an $n=6$ and $n=7$ mode instability are shown in Figs. 35 and 36 . The actual observed mode number $n$ of the instability is shown in Fig. 37 as a function of $\tilde{V}=U_{0} / \Gamma_{0} / 4 \pi r_{0}$. The LDV system was used to determine $\Gamma_{0}$, and $U_{0}$ and $r_{0}$ were measured photographically. Also shown is the value of $n$ that is predicted to be the most unstable for that value of $V$. Although the trend of increasing $n$ with increasing $\widetilde{V}$ (decreasing core size) is in agreement with the theoretical preaictions, the actual values of $n$ vs $\tilde{V}$ are not in good agreement. This is most likely due to the fact that the ratio of wave length to vortex core radius of the unstable mode is not small as required by the asymptotic theory. The measured radial perturbation of the ring due to the instability as a function of time is shown in Fig. 38. The growth of the perturbation during the early stages of the instability shows good agreement with the 
55

theoretical predictions. This is due in part to the fact that the amplification rate for an unstable mode turns out to be insensitive to both $\widetilde{V}$ and modal number $n$. 
CHAPTER V

HELICOPTER ROTOR WAKE

5.1. Introduction

The wake of a helicopter rotor is another example of a flow field where the use of conventional instrumentation is hampered by probe interference and calibration in a rapidly changing velocity field. Hollow rotor blades were constructed so that smoke could be injected at any point along the blade to provide flow visualization and particles for the LDV. From the velocity measurements the bound circulation and hence the load distribution along the rotor are found.

5.2. Construction of a Rotor for Use with LDV System

A rotor stand capable of spinning a one or two bladed model motor was designed and constructed. The hollow rotor blades permit smoke to be deposited at the tip or any other desirable place along the blade.

A cross section of the blade in Fig. 39 shows the epoxyfiberglass structure with a stainless steel spar, berylliumcopper inner chamber and a tungsten rod to maintain balance about the quarter chord. Initially an aluminum model was machined from which a mold (Fig. 40) was cast of stycast 2850, a high strength casting resin.

The beryllium-copper was spot-welded to the stainless steel spar and the tungsten rod silver soldered on the front of it. An 
inner mandrel was inserted inside the beryllium-copper and the outside wrapped with fiberglass wet with Eccomold L-28 laminating resin. This structure was compressed in the mold to form the NACA-0012 airfoil section. After the epoxy cured the blade was removed from the mold and the inner mandrel removed. The resulting hollow blades are shown in Fig. $40 \mathrm{~b}$ and $40 \mathrm{c}$.

The arive stand for the rotor (Fig. 4l) has a hollow shaft to allow smoke to pass to the rotor. The shaft is driven by an 1800 rpm synchronous motor.

Table 3 lists the relevant parameters for the single bladed rotor used throughout this experiment.

\subsection{Flow Visualization of Rotor Wake}

Several interesting features of the flow field in the wake of the model rotor were observed using smoke flow visualization. The coordinate system usea for describing the rotor experiments is shown in Fig. 42 .

A side view strobe picture of the tip vortex (Fig. 43) shows an unexplained wavelike structure. Oscillations in the tip vortex of a single bladed rotor are mentioned by Ref. 43 but no photographs or explanation are given. Attempts have been made to explain this wave using the analysis of the stability of a helical vortex by Widnall (Ref. 44), but the phase of the observed wave is different than the theory predicts. This is probably due to the fact that the theory does not include the effects of the inboard vortex sheet.

When smoke is released upstream of the rotor plane, it becomes entrained in the core of the tip vortex and travels up 
the core towards the rotor blade as shown in Fig. 44 indicating a strong axial flow in the vortex. Since flow along the core of a vortex affects the self-induced motion this may be important in analytic determination of vortex position. A slit was cut in the top of the blade at the quarter chord between $r /$ Ro $=.82$ and $r /$ Ro $=.94$. The results of releasing smoke through this slit and at the tip (Fig. 45), show that the sheet of smoke does not immediately roll up into the tip vortex. Additional observations inaicate that it takes over a complete revolution before the sheet is rolled up.

In order to obtain the position of the tip vortex, smoke was ejected at the tip of the blade and the wake illuminated with a narrow plane of light. From a series of photographs, examples of which are shown in Fig. 46, the tip vortex position is established. The coordinates of the tip vortex given in Figs. 47 and 48 are in general agreement with the averaged experimental results of Ref. 43 .

A photograph looking along the positive $z$ axis from beneath the rotor, Fig. 49, shows some interesting features of the tip vortex. It can be seen that the tip vortex gradually increases in diameter until regions of sudden enlargement (vortex breakdown) are encountered. A graph of core diameter vs. azimuthal angle, Fig. 50, shows this increase.

The two component laser Doppler velocimeter (LDV) described in Chapter II was used to measure velocities around the hovering model rotor blade. Two separate measurement setups were used to determine all three components of the velocity 
vector. Several examples of the velocity data are shown in Fig. 51 for a single untwisted blade at an angle of attack $\theta_{0}=10^{\circ}$. The curves show velocity vs. time as the rotor blade passes a fixed point. The sign of the velocity component is not given by the LDV system, due to an inherent $180^{\circ}$ ambiguity, and must be determined by independent means such as flow visualization. The proper sign of the velocity component is indicated for each data curve. Fig. 51 a-f are on a short enough time scale so that details of the flow field in the immediate vicinity of the blade are shown. Fig. 51 e shows the downwash $v_{z}$ for a complete revolution of the blade. Note that the data below the blade (Figs 5lb, $d$, and $f$ ) in the relatively small region of the viscous wake are extremely unrepeatable.

By integrating the velocity data along the contour shown in the dotted lines in Fig. 42, the bound circulation and hence the section load can be determined. The entire load distribution is found by performing the integration at a series of radial locations. The results of these integrations are shown in Figs. 52 and 53. From blade element theory (Ref. 45) for an untwisted rotor

$\frac{\Gamma(x)}{\left(\Omega R_{0}\right) \pi R_{0}}=\frac{\sigma a}{2}\left[\theta_{0} x-\frac{\sigma a}{16}\left(-1+\left(1+32\left(\frac{\theta_{0}}{\sigma a}\right) x\right)^{1 / 2}\right)\right]$ 
and $\frac{d C_{T}}{d x}=\chi\left(\frac{\Gamma(x)}{\left(\Omega R_{0}\right) \pi R_{0}}\right)$

where

$$
\begin{aligned}
\Gamma(\mathrm{x}) & =\text { bound circulation at } \mathrm{x} \\
\mathrm{x} & =\text { r/Ro } \\
\text { Ro } & =\text { radius of rotor } \\
\Omega & =\text { angular velocity } \\
\sigma & =\text { solidity } \\
\theta_{0} & =\text { angle of attack } \\
\mathrm{a} & =\text { slope of the lift curve } \\
\mathrm{C}_{\mathrm{T}} & =\text { thrust coefficient }
\end{aligned}
$$

The comparison between blade element theory and experiment shows the expected discrepancy at the tip since the theory does not include the effect of the tip vortex. When a tip loss factor of .96 is applied to the theory, a value of $\mathrm{C}_{\mathrm{T}}=.00388$ is obtained which is within $1.8 \%$ of the value obtained from an overall force balance. A value of $\mathrm{C}_{\mathrm{r}}=.00357$ is obtained from the laser data by finding the area under the experimental curve of Fig. 53. Although this is in error by 6.5\%, increased accuracy could be obtained by taking more data and averaging and by improving the accuracy of the Doppler frequency measurement. Also the method of obtaining the local lift from $p U(x) I(x)$ is in error in the tip region. The load distribution of Fig. 53 and the tip vortex position of Figs. 47 and 48 offers a relatively simple test case for any free vortex wake analysis of a hovering rotor. 
5.4. LDV Measurements Near the Tip Vortex

Numerous attempts were made to obtain data in the tip vortex of the model rotor. Examples of LDV data taken at a position strongly influenced by but not directly within the tip vortex are shown in Fig. 54. The point of closest approach of the vortex to the measuring volume occurs at approximately $50^{\circ}$ behind the quarter chord. The data are now beginning to show more scatter. When the probe is moved closer to the tip vortex the scatter renders the data uselsss. Since the LDV data near the blade are repeatable the cause of scatter must be due to fluctuations of the tip vortex position. In order to obtain information about the structure of the tip vortex an electronics system that does not require the flow field to be periodic is needed. It is also recommended that a larger model rotor be used so that the reynolds number would be higher and the velocity gradients across the probe smaller. 


\section{CHAPTER VI \\ CONCLUSIONS AND RECOMMENDATIONS}

\subsection{Summary and Conclusions}

The use of conventional instrumentation such as a hot wire anemometer for measuring velocity in vortex filament is hampered by probe interference and the difficulty of calibrating the probe in a flow field where the velocity is changing rapidly in magnitude and direction. For these reasons a laser Doppler velocimeter (IDV) is used throughout these experiments. The IDV measures the velocity of smoke particles by measuring the Doppler shift of laser light scattered from the particles. The LDV system designed and constructed is a dual scatter system capable of determining two components of the velocity vector. The polarization of the incident beams used for one component is perpendicular to that for the other component to prevent cross talk and allow the components to be separated. By using conventional prism-type beamsplitters a compact in line system that maintains equal path lengths for all beams has been constructed.

The most unique feature of the LDV system is the electronics system which is capable of handling the large, fast changes in velocity which are encountered in the flow fields of interest. The electronics system described in section 2.5 requires that the flow field be periodic. It is a "sampling" system in that only a few points at a particular phase on the velocity-versus-time curve are determined 
per cycle. By varying the phase the remaining points are determined. The system requires only a spectrum analyzer for demodulation and a storage oscilloscope for retaining the data.

The main arawback to the LDV is the fact that the velocity measured is that of smoke particles and not the fluid velocity. Therefore it is necessary to assure that the particle size is small enough so its velocity is nearly equal to the fluid velocity. This is especially true in the present experiments where oil particles with a density of $10^{3}$ times that of air are used. The oil particles are generated by a standard mist lubricator--a device normally used to lubricate high speed bearings. The particles have a diameter of approximately $1.0 \mu$ which was determined by a light scattering experiment that measured the shape of the forward scattering lobe. It is estimated that oil particles of this size will follow the fluid velocities encountered in these experiments within $1.0 \%$.

A detailed study of the structure and stability of vortex rings using the two-component LDV system has been made. Vortex rings were generated by pulsing air through a sharp-edged orifice using a loud speaker. The circulation and core size are controlled by the amplitude and duration of the electric signal applied to the loud speaker.

Detailed surveys of two vortex rings were made; a relatively thick core ring with a ratio of core radius to ring raaius $a / r_{0}=.27$, and a relatively thin core ring with $\mathrm{a} / \mathrm{r}_{\mathrm{o}}=.075$. The principal result, Fig.31, shows that the vorticity is concentrated in a small core region but that the core is 
not in solid body rotation. The self-induced velocity predicted by theory for the measured vorticity distribution is in error by $20 \%$, probably due to the fact that $a / r_{0}=.27$ is not small enough for the theory to be valid. (only the thick core case could be compared.)

During the course of the investigation, the fact that vortex rings are unstable was rediscovered. Azimuthal perturbations with between 6 and 16 waves spontaneously develop on the perimeter of the ring and continue to grow. For a given vortex core, only one mode is unstable and the smaller the vortex core, the larger the number of waves in the mode. Theoretical predictions of the mode number are not good, but the correct trend is indicated. The amplification rate is accurately predicted probably because all modes have about the same amplification rate.

A single bladed model helicopter rotor has been constructed. The blade is hollow to allow smoke to be deposited at the tip or any desirable point along the span providing flow visualization and scattering particles for the LDV system. The flow visualization data in hover show an unexplained wavelike structure on the helical tip vortex. Also a significant axial velocity up the core of the tip vortex towards the blade is evident from the smoke photographs.

Velocities around the hovering blade were measured with the IDV system. By making two separate measurement setups, all three components of the velocity vector were determined. From this data the bound circulation and hence the spanwise load 
distribution was found. Attempts at using the LDV system to measure the structure of the tip vortex were largely unsuccessful due to random fluctuations in the position of the vortex which could not be handed with the present electronics system.

\subsection{Recommendations}

In order to improve velocity measurements in vortices within an IDV it is first necessary to develop fast electronics that can handle the large and rapid changes in Doppler frequency that are encountered. Although the presently available frequency trackers are not fast enough, their response to large fluctuations could be improved by increasing the I.F. frequency. If the I.F. frequency is made much larger than the changes in Doppler frequency then the time response should be improved.

Once adequate electronics is developed it is necessary to overcome the problem of random fluctuations in the position of the vortex. This could be done by rapidly moving the measuring volume of the LDV through the vortex. This scan must be fast compared to the fluctuation speed of the vortex so that the vortex position is essentially steady during the measurement. Ultimately what is needed is a three-dimensional raster scan of the volume containing the vortex. While the method of producing such a scan is not completely clear, it would appear that commercially available pizeoelectric or electromagnetic scanners should be adequate.

The study of vortex rings could be expanded using the LDV setup without modification. An investigation of the rollup and 
formation process would be particularly interesting. The present investigation could also be extended to include rings with smaller cores, and the effects of buoyancy.

The use of the improved LDV system described above for measurements of the flow field around the helicopter rotor is the most practical and pressing problem. The use of a larger model rotor would increase the Reynolds number and also the size of the tip vortex compared to the probe volume. With this system the structure and position of the tip vortex and the load distribution could be found. 
REFERENCES

1. Yeh, Y., and Cummins, H. Z., "Localized Fluid Flow Measurements with an He-Ne Laser Spectrometer," Applied Physics Letters, Vol. 4, No. 10, 1964.

2. -- Bibliography of Laser Doppler Anemometer Literature, Disa--S and B, Inc., Franklin Lakes, N. J., 1972 .

3. Stevenson, W. H., Pedigo, M. K., Zemmit, R. E., "Bibliography on Laser Doppler Velocimeters", Laser Doppler velocimeter Workshop, Puraue University, 1972.

4. Goldstein, R. J. and Kreia, D., K., "Measurement of Laminar Flow Development in a Square Duct Using a Laser-Doppler Flowmeter," Journal of Applied Mechanics, December 1967.

5. Ruda, M. J. "A New Theoretical Model for the Laser Dopplermeter," J. Sci. Instrum. (J. Phys E.), Series 2, Vol. 2 pe 55-58, 1969.

6. Rogers, $W . B ., "$ On the Formation of Rotating Rings of Air and Liquids Under Certain Conditions of Discharge," American Journal of Science and Arts, Vol. 26, pp 247-259, 1858 .

7. Helmholtz, H., "Ueber Integrale der Hyarodynamischen Gieichungen welche den Wirbelbewegungen entsprechen, Crell, 1858, translated by Tait in Phil. Mag., Vol. XXXIII(1867).

8. Thomson, W. A., "On Vortex Atoms," Phil. Mag. Vol. XXXIV, 1867 .

9. Reynolds, 0., "On the Resistance Encountered by Vortex Rings, and the Relation Between the Vortex Rings and the Streamlines of a Disc," lvature, Vol. 14, pp 477-479, 1876 .

10. Thomson, J. J., A Treatise on the Motion of Vortex Rings, MacMilian and Co., London, 1883.

11. Hicks, W. M., "Researches on the Theory of Vortex Rings-Part II", Philosophical Transactions of the Royal Society of London, Vo1. 176, Pt. 2, pp 725-780, 1885. 


\section{REFERENCES (continued)}

12. Dyson, F. W., "The Potential of an Anchor Ring," Phil. rrans. A. CLXXXIV, 1893.

13. Pocklington, H. C.," "The Complete System of the Periods of a Hollow Vortex Ring," Philosophical Transactions of The Royal Society of London, Vol. 186, Pt. 2, pp 603-619, 1895.

14. Hill, M. J. M., "On a Spherical Vortex," Philosophical Transactions of the Royal Society of London, Vol. 185, Pt. I, pp 213-245, 1894 .

15. Northrup, E. F., "An Experimental Study of Vortex Motions in Liquids," Journal of the Franklin Institute, Vol. CLXXII, No. 3, p 211 and $345,1911$.

16. Sadron, M., "Contribution a l'Etude de la Formation et de la Propagation des Anneaux de Tourbillion dans l'Air," Journal de Physique, Vol. 7, No. 3, pp 76-91, 1926.

17. Banerji, $K$. , and Barave, R. V., "On Oberbeck's Vortices," Phil. Mag., Vol. 11, pp 1057-1081, 1931.

18. Krutzsch, C. H., "Uber eine experimentell beobachtete Erscheining an Werbelringen bei ehrer translatorischen Beivegung in Werklechin," Flussigheiter Annalen Der Physik 5, Folge Band 35, pp 497-523, 1939.

19. Turner, J. S., "Buoyant Vortex Rings," Proceedings of the Royal Society of London, A., Vol. 239, pp 61-75, 1957.

20. O,Brien, Vivian, "Steady Spheroidal Vortices--More Exact Solutions to the Navier-Stokes Equation," Quarterly of Applied Mathematics, Vol. XIX, No. 2, July 1961.

21. Margavey, R. H. and Maclatchy, C. S., "The Formation and Structure of Vortex Rings," Canadian Journal of Physics, Vol. 42, April 1964.

22. Zaroodny, S. J., "Revised Theory of Vortex Rings--A Simplified Review of the State-of-the-Art,", U. S. Army Limited War Laboratory, TM 66-01, 1966.

23. Tung, $O$. and Ting, $L$., "The Motion and Decay of a Vortex Ring," Physics of fluids, Vol. 10, No. 5, pp 901-10, May, 1967. 


\section{REFERENCES (continued)}

24. Widnall, S. E., Bliss, D. B., and Zalay, A., "Theoretical and Experimental Study of the Stability of a Vortex Pair," Proceedings of the Symposium on Aircraft Wake Turbulence, Seattle, Washington, 1970.

25. Fraenkel, L. E., "On Steady Vortex Rings of Small Cross Section in an Ideal Fluid," Proceedings of the Royal Society, A316, p 29.

26. Saffman, P. G., "The Velocity of Viscous Vortex Rings," Studies in Applied Math., Vol. XIIX, No. 5, pp 370-380, 1970 .

27. Johnson, G., "Researches on the Propagation and Decay of Vortex Rings," ARI 70-0093, June 1970.

28. Kambe, T. and Toshihara, T., "Motion of Distorted Vortex Rings," J. of the Physical Soc. of Japan, Vol. 31, No. 2, July 1971.

29. Oshima, Y., "Motion of Vortex Rings in Water," J. of the Physical Soc. of Japan, Vol. 32, No. 4, April 1972.

30. Maxworthy, T., "The Structure and Stability of Vortex Rings," J. Fluid Mechanics, Vol. 51, No. 1, p 15, 1972.

31. Fraenke1, L. E., "Examples of Steady Vortex Rings of Small Cross Section in an Ideal Fluid," JFM 5111972

32. Scully, M. P., "A Method of Computing Helicopter Vortex Wake Distortion," MIT Aeroelastic and Structures Research Laboratory, TR138-1, June 1967.

33. Landgrebe, Anton T., "An Analytical and Experimental Investigation of Helicopter Rotor Hover Performance and wake Geometry Characteristics," USAAAMRDI Tech. Rpt. 71-24, June 1971 .

34. Chigier, N. A. and Corsiglia, V. R., "Tip Vortices-Velocity Distributions," 27th Annual National V/STOL Forum of the American Helicopter Society, Preprint 522, May 1971.

35. Rorke, J. B., Moffitt, R. C. and Ward, J. F., "Wind Tunnel Simulation of Full Scale Vortices," 28th Annual National Forum of the American Helicopter Society, Preprint 623, May 1972. 


\section{REFERENCES (continued)}

36. Cook, C. V., "The Structure of the Rotor Blade Tip Vortex," AGARD-CPP-IIl, Conference on Aerodynamics of Rotary Wings, September 1972 .

37. Lennert, A. E., and Brayton, E. B., and Crosswy, F. L., Summary Report of the Development of a Laser Doppler

Velocimeter to be Usea in AEDC Wind Tunnels, AEDC-Tr-70-101, 1970 .

38. Born, M. and Wolf, E., Principles of Optics, Pergammon Press, 1970 .

39. Iten, P., Private Communication.

40. Hodkinson, J. R., "Particle Sizing by Means of the Forward Scattering Lobe," Applied Optics, Vol. 4, No. 5, May 1966.

41. Brodkey, P. S., The Phenomena of Fluid Motions, AddisonWesley Publishing Company, 1967.

42. Wianall, S. E. and Sullivan, J. P., "On the stability of Vortex Rings," to be published in Proceedings of the Royal Society, Series A.

43. Gray, R. B. and Brown, G. W., "A Vortex Analysis of SingleBladed Hovering Rotor and a Comparison with Experimental Data," AGARD-CPP-lli, Conference on Aerodynamics of Rotary Wings.

44. Widnall, S. E., "The Stability of a Helical Vortex Filament," JFM, 54, 4, pp 641-663.

45. Gessow, A. and Myers, G. C., Jr., Aerodynamics of the Helicopter, MacMillan Co., New York, 1952.

46. Innes, D. J., and Bloom, A. L., "Design of Optical Systems for Use with Laser Beams," Spectra-Physics Laser Technical Bulletin No. 5 . 


\section{TABLE 1}

\section{LDV Characteristics}

$$
\begin{aligned}
& \text { Laser - HeNe Spectra Physics Model } 124 \\
& \lambda=6328 \AA \\
& \mathrm{P}_{\mathrm{L}}=15 \mathrm{~mW} \\
& 2 \mathrm{~b}=3.8 \mathrm{rm} \text { (at focusing lens) } \\
& f=12 \mathrm{in.}=304.8 \mathrm{~mm} \text { (focusing lens) } \\
& \theta_{\mathrm{B}} / 2=.0681 \text { Rad. }=3.90^{\circ} \\
& f_{D}=\left(6.5716 \times 10^{4} \mathrm{U}\right) \mathrm{Hz} \text { (where } U \text { is the velocity in } \mathrm{ft} / \mathrm{sec} \text { ) } \\
& b_{0}=\frac{2 F \lambda}{\pi}=.032 \mathrm{~mm}=.0013^{\prime \prime} \\
& \text { Diameter of probe volume } \simeq 2 \mathrm{~b}_{\mathrm{O}}=.0026^{\prime \prime} \\
& \text { Length of probe volume } \simeq 2 \mathrm{~b}_{\mathrm{O}} / \sin \theta_{B} / 2=.038^{\prime \prime} \\
& \text { Volume of probe }=2.02 \times 10^{-6} \mathrm{~cm}^{3} \\
& \text { Particles are made from "Fog Juice" (Iight oil) } \\
& \alpha=\pi d / \lambda=5.0 \\
& \mathrm{~N}=8.5 \times 10^{6} \text { particles } / \mathrm{cm}^{3}
\end{aligned}
$$


TABLE 2 DATA SUMMARY

$$
r_{0}(f t) \quad U_{0}(f t / \sec ) \quad \Gamma_{0}\left(f t^{2} / \mathrm{sec}\right) \quad a / r_{0} \quad \tilde{V}^{*} \quad \ln 8 r_{0} / a-I / 4^{*} \quad R e=\Gamma / \nu
$$

$\begin{array}{llllllll}\text { Ring No. } 1 & 0.091 & 2.60 & 1.21 & 0.27 & 2.46 & 3.14 & 7780 \\ \text { Ring No. } 2 & 0.111 & 13.5 & 6.07 & 0.075 & 3.10 & 4.42 & 37900\end{array}$

\footnotetext{
${ }^{*}$ Measured $\widetilde{V}=U_{0} / \Gamma_{0} / 4 \pi r_{0}$.

** Value of $\widetilde{V}$ from theory with solid body rotation core.
} 
Table 3

Model Rotor Parameters (Single Blade)

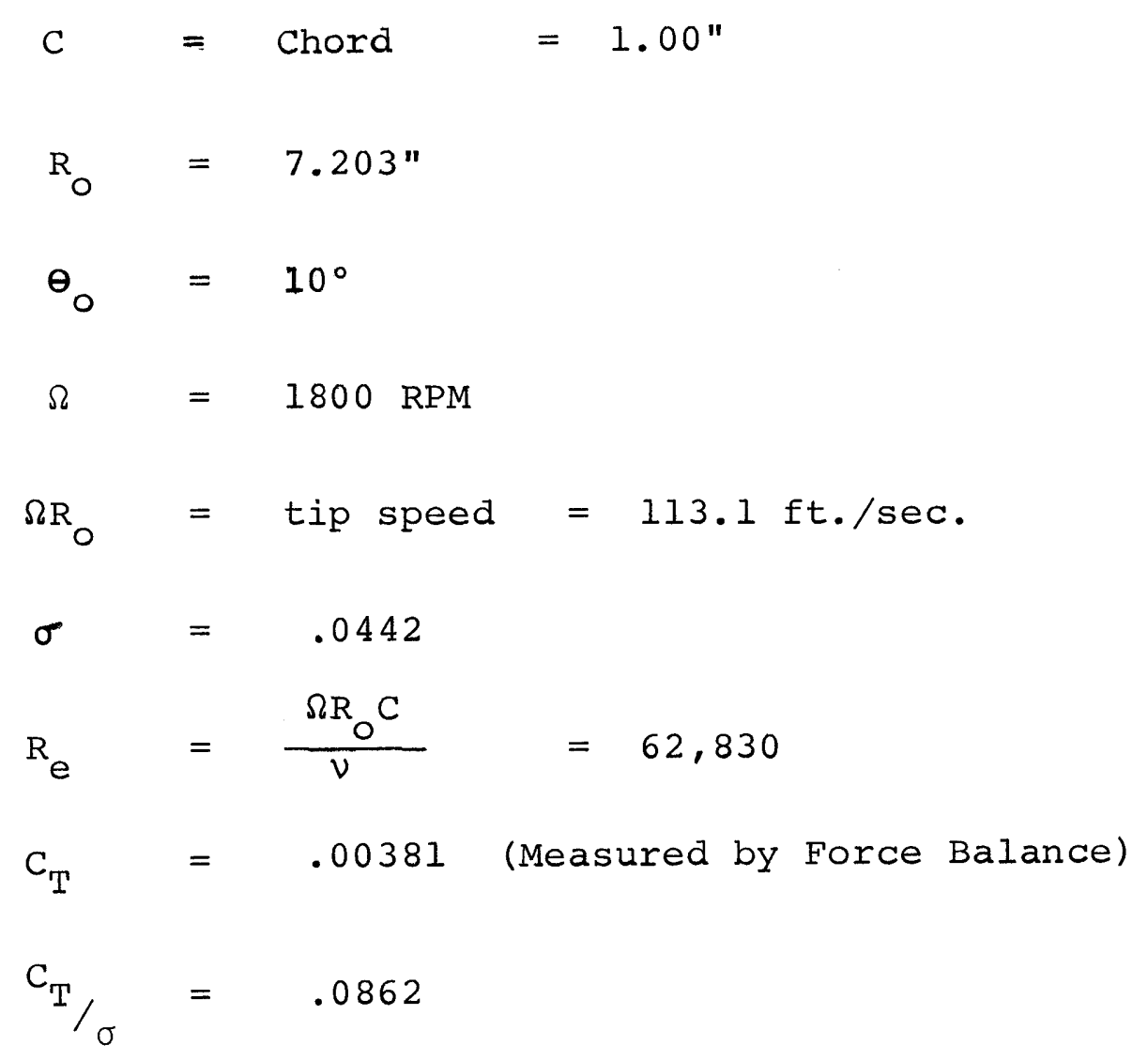


74

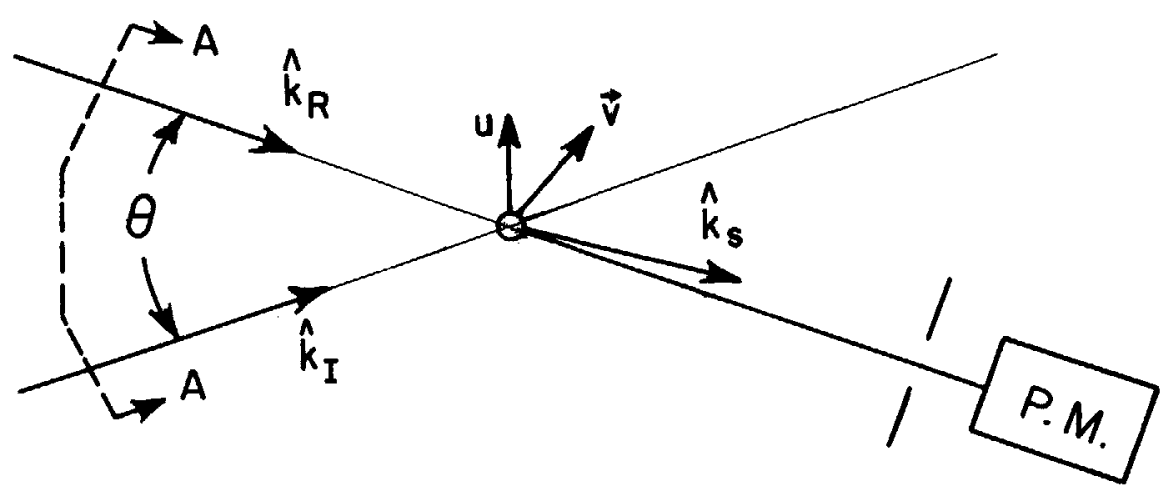

REFERENCE BEAM SYSTEM

Fig. Ia
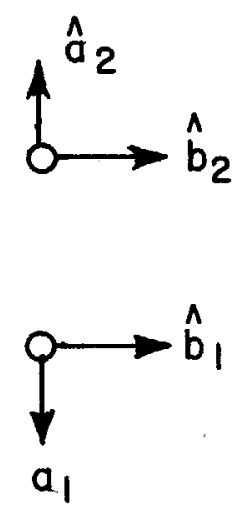

SECTION AAA

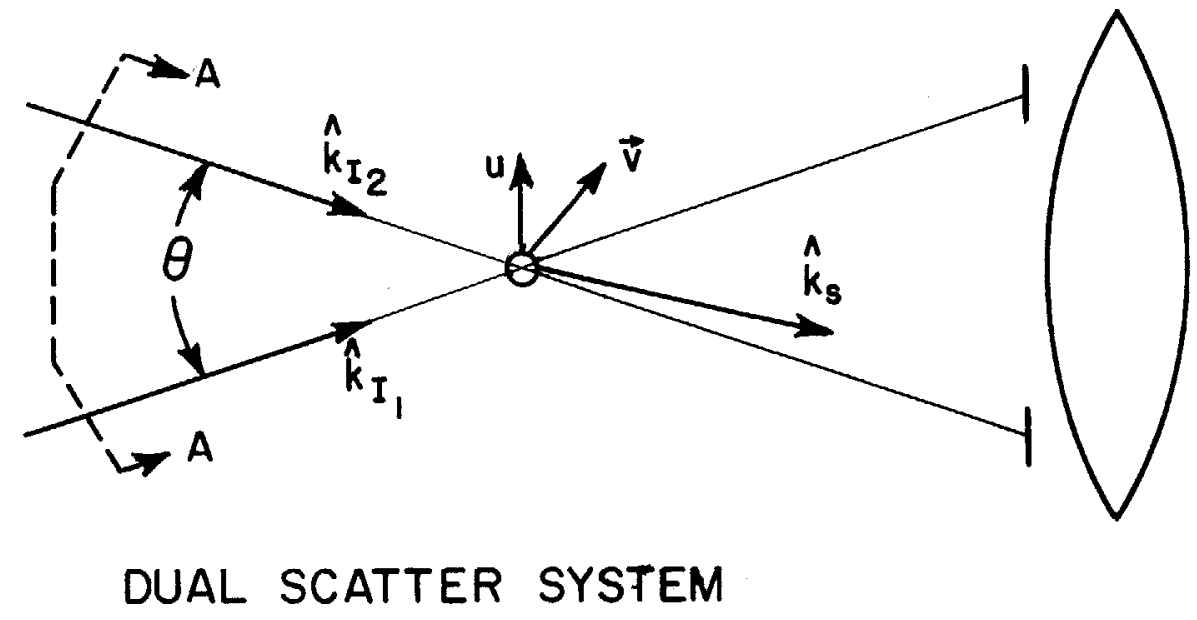

Fig. $1 b$ 


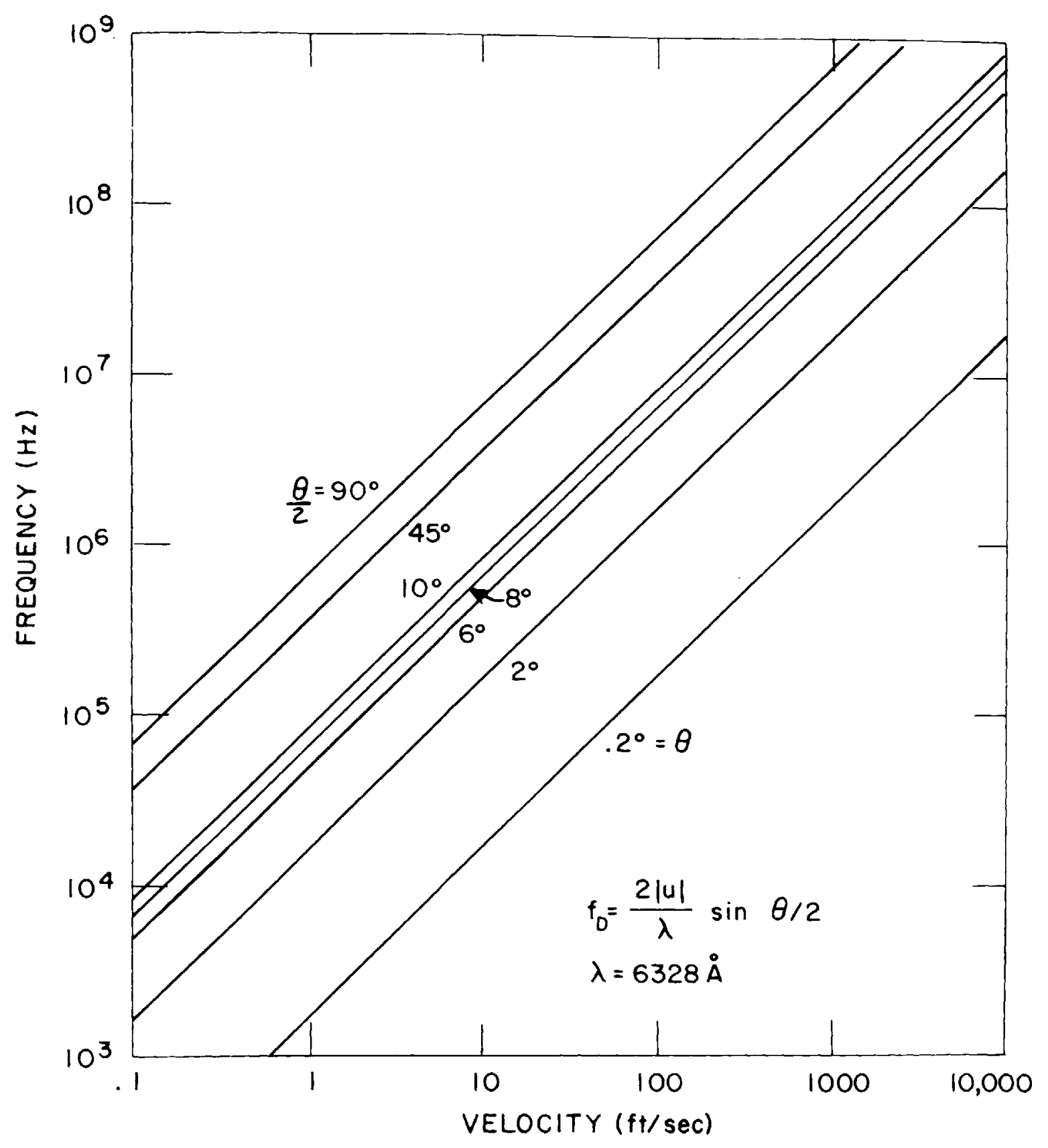

Fig. 2. Doppler shift vs. velocity for He-Ne laser at various scattering angles. 


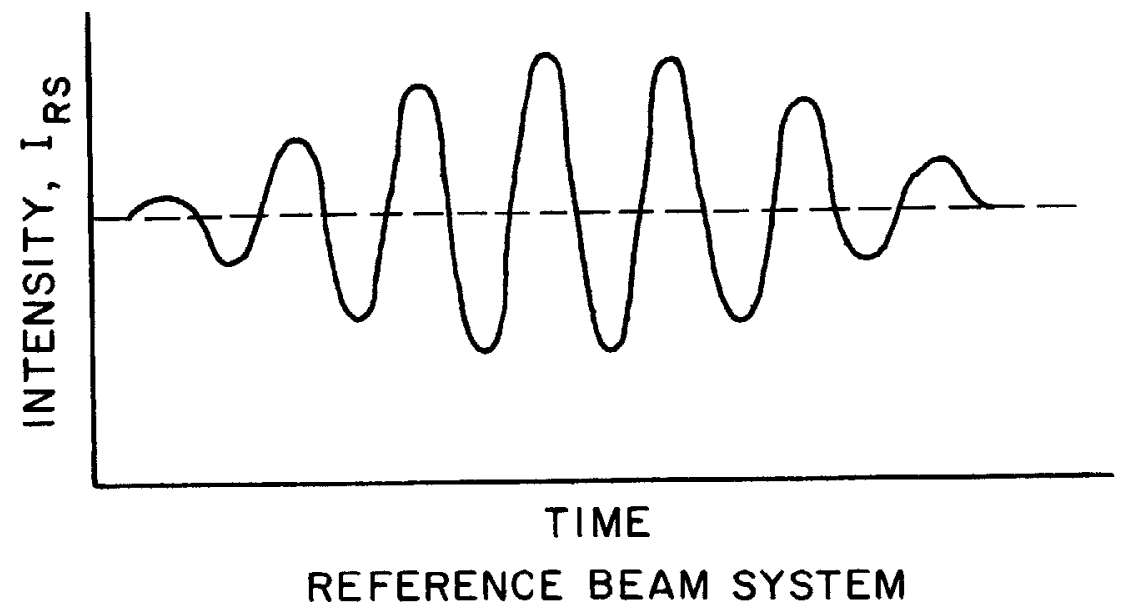

Fig. $3 a$

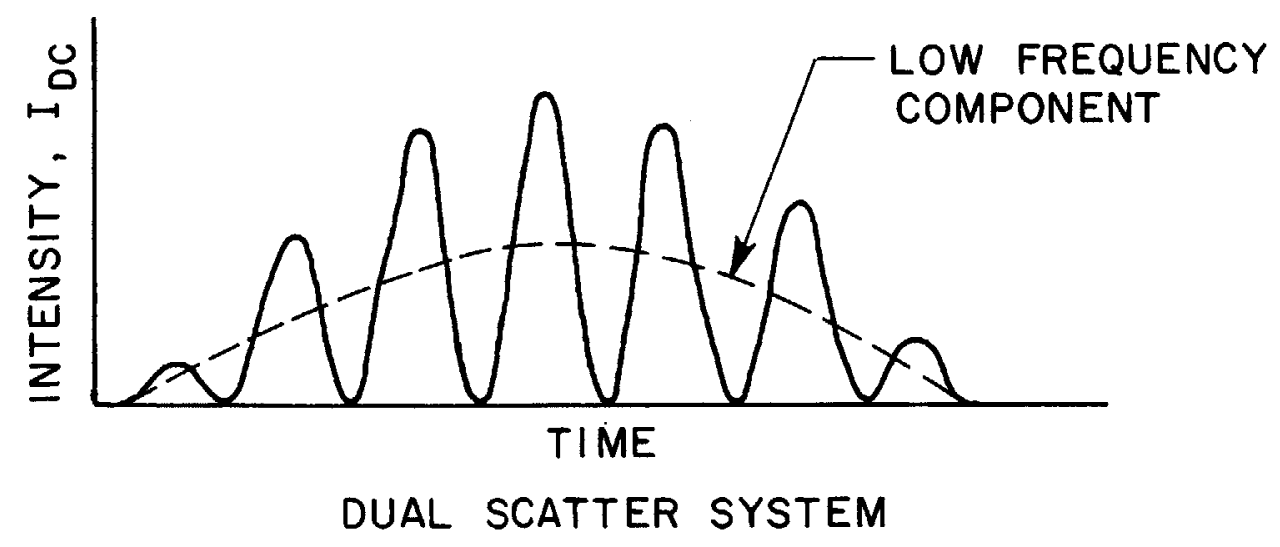

Fig. $3 b$ 


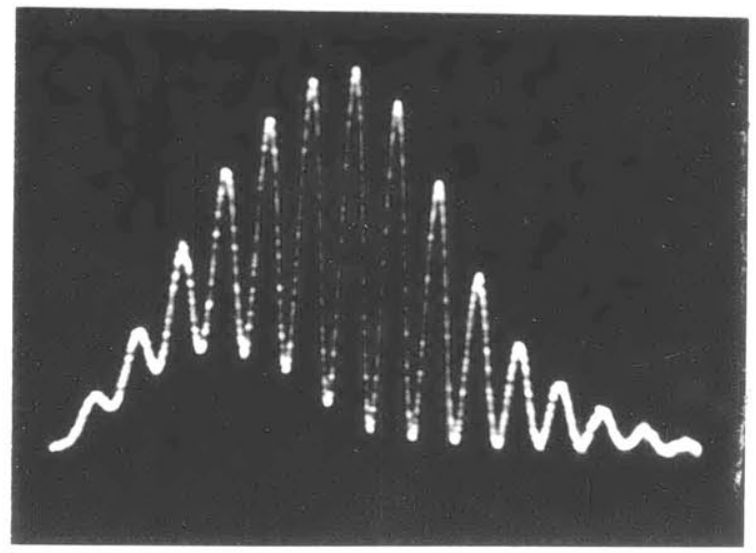

Fig. 4a. Doppler signal from .0002 dia. wire passing through center of probe volume. Vert.scale $100 \mathrm{MV} / \mathrm{cm}$ Horiz." . $\mathrm{MS} / \mathrm{cm}$

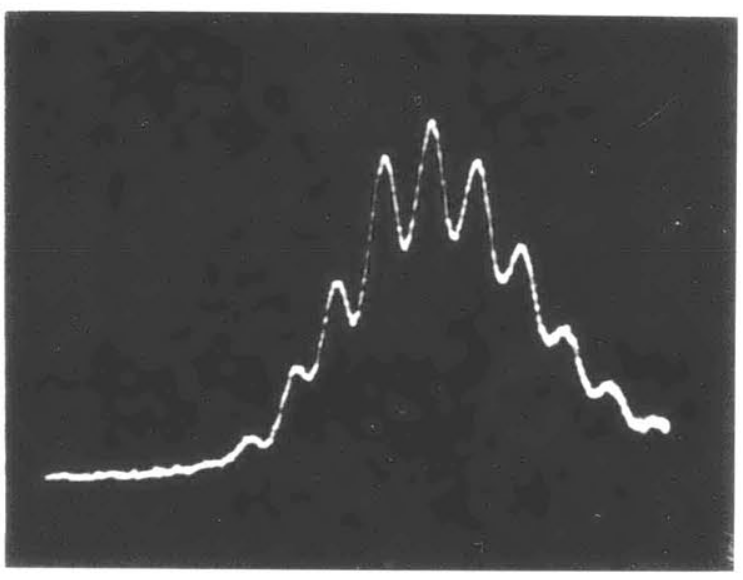

Fig. 4b. Doppler signal from .0002 dia. wire which did not pass through center of probe vol. scale same as $4 a$.

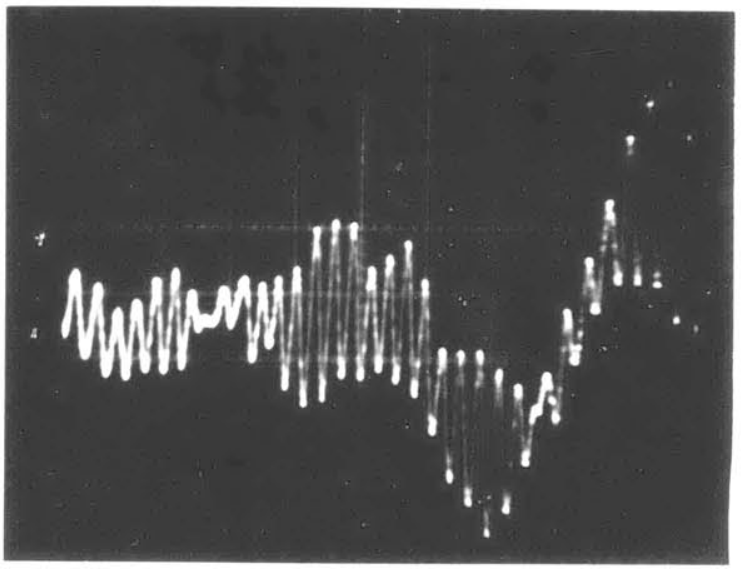

Fig. 4c. Doppler signal from spinning disk.

Vert.scale $100 \mathrm{MV} / \mathrm{cm}$ Horiz. " $50 \mathrm{~ms} / \mathrm{cm}$ 
REFERENCE BEAM

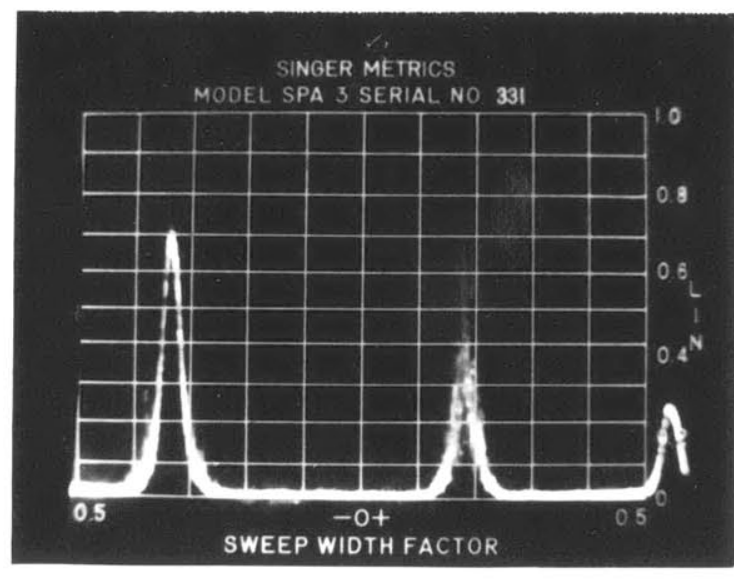

0

$500 \mathrm{KHZ}$
1. Disk

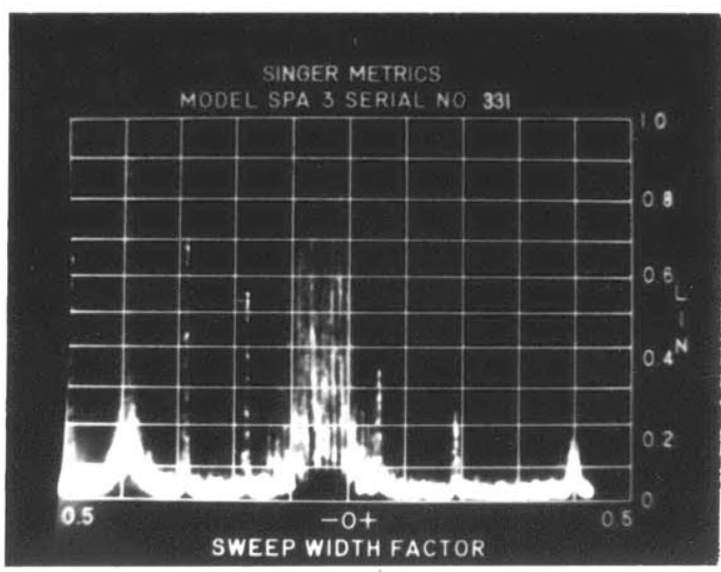

$0 \quad 1 \mathrm{MHZ} \quad 2 \mathrm{MHZ}$

2. Smoke Jet
DUAL SCATTER

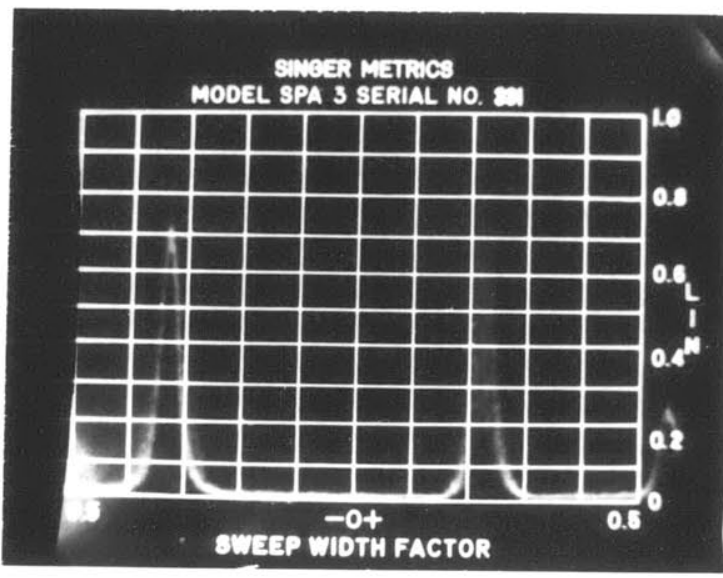

0

$500 \mathrm{KHZ}$

1. Disk

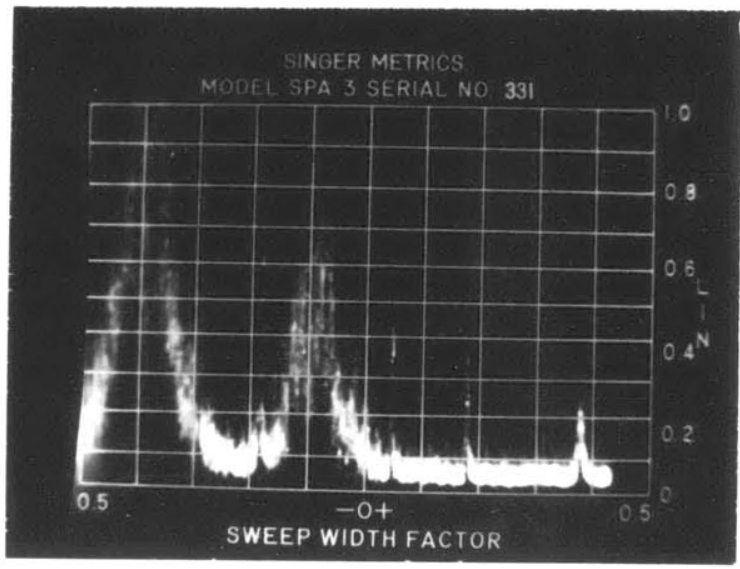

$0 \quad 1 \mathrm{MHZ} \quad 2 \mathrm{MHZ}$

2. Smoke Jet

Fig. 5. Signals from Ref. Beam \& Dual Scatter systems 


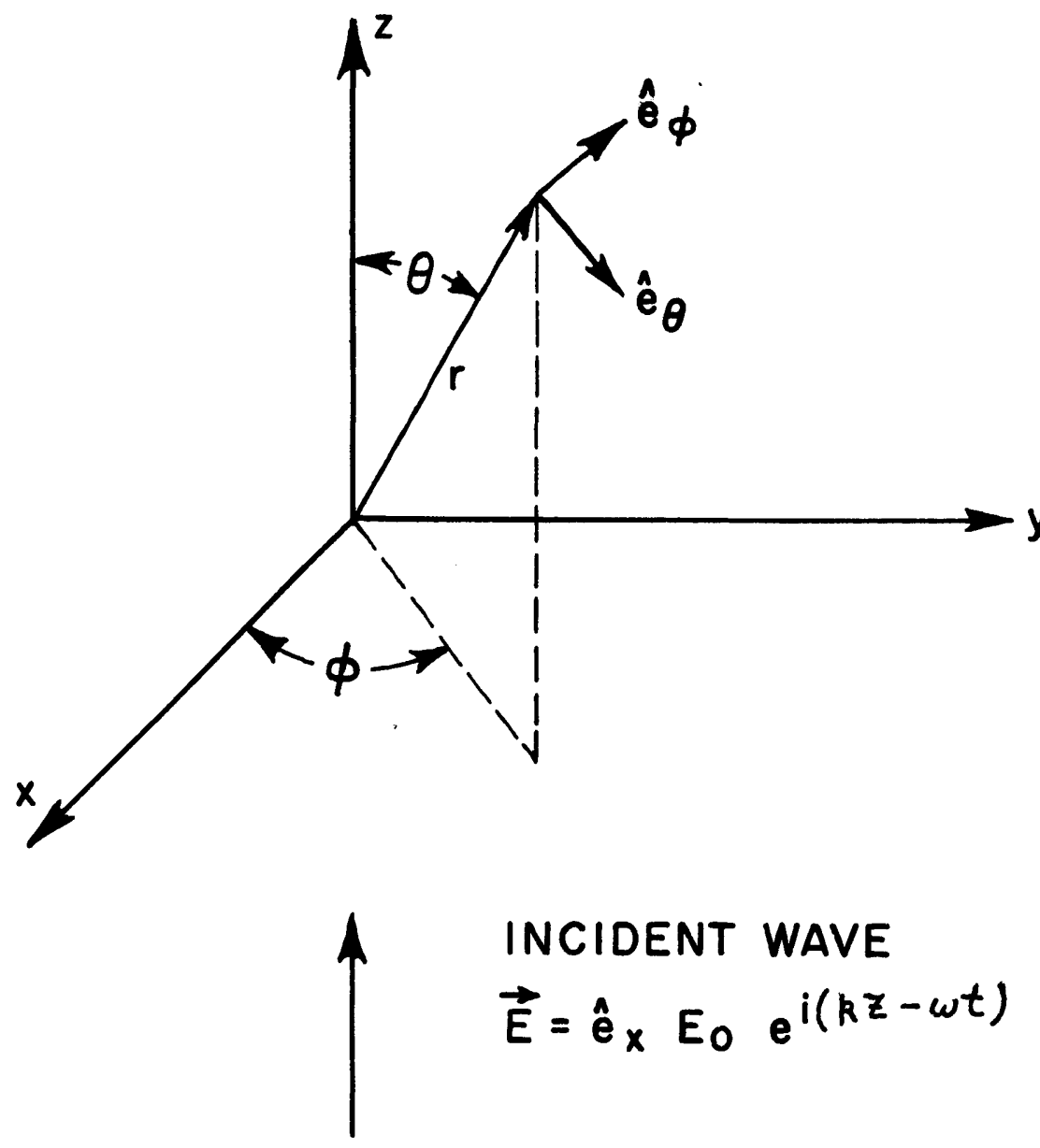

Fig. 6. Coordinate System for Mie Scattering 


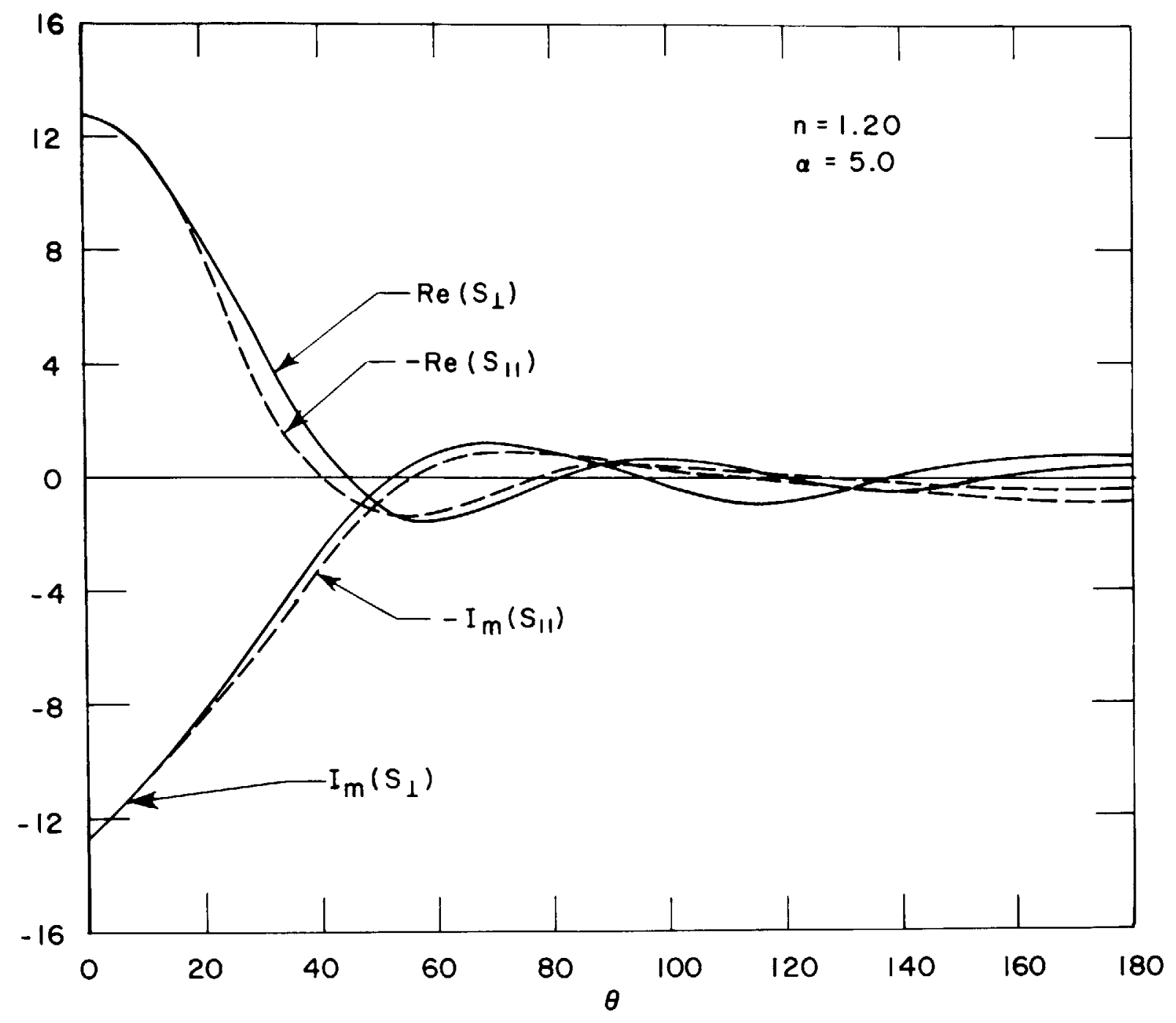

Fig. 7. Real and Imaginary parts of $\mathrm{S}$ 


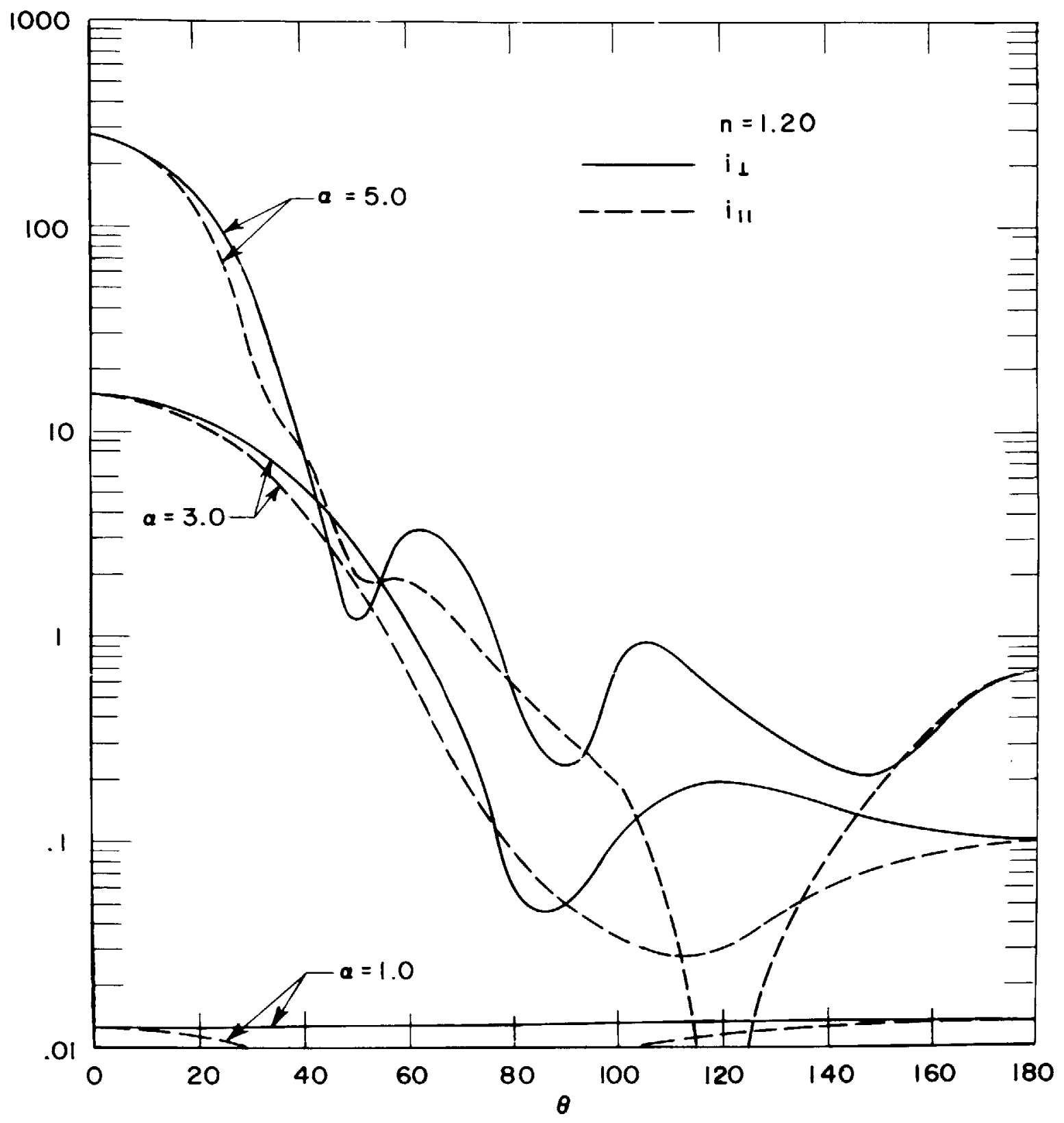

Fig. 8. Scattering Intensity Functions 


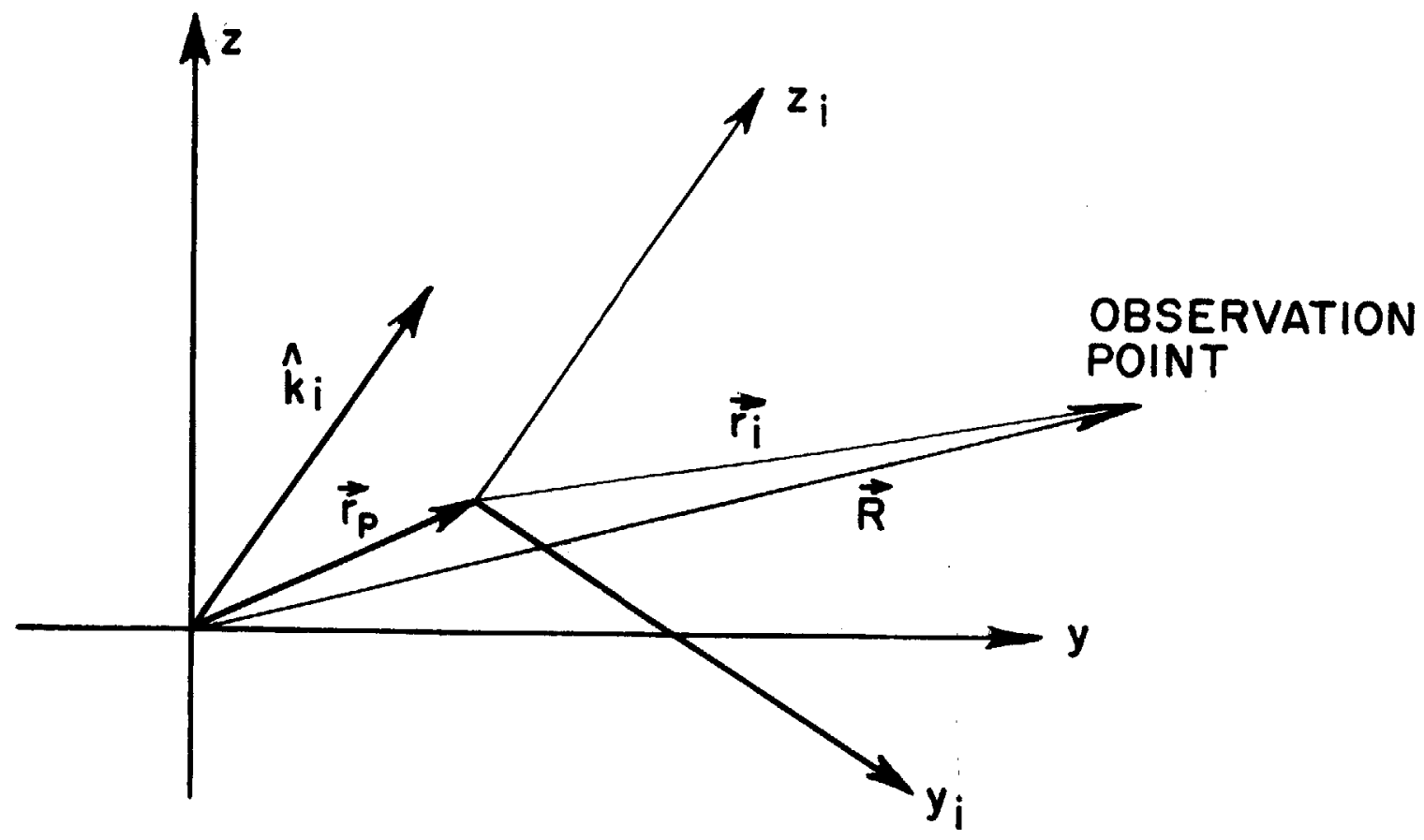

Fig. 9. Coordinate System for scattering of Incident Wave in $\hat{k}_{i}$ Direction by Particle Located at $\vec{r}_{p}$ 


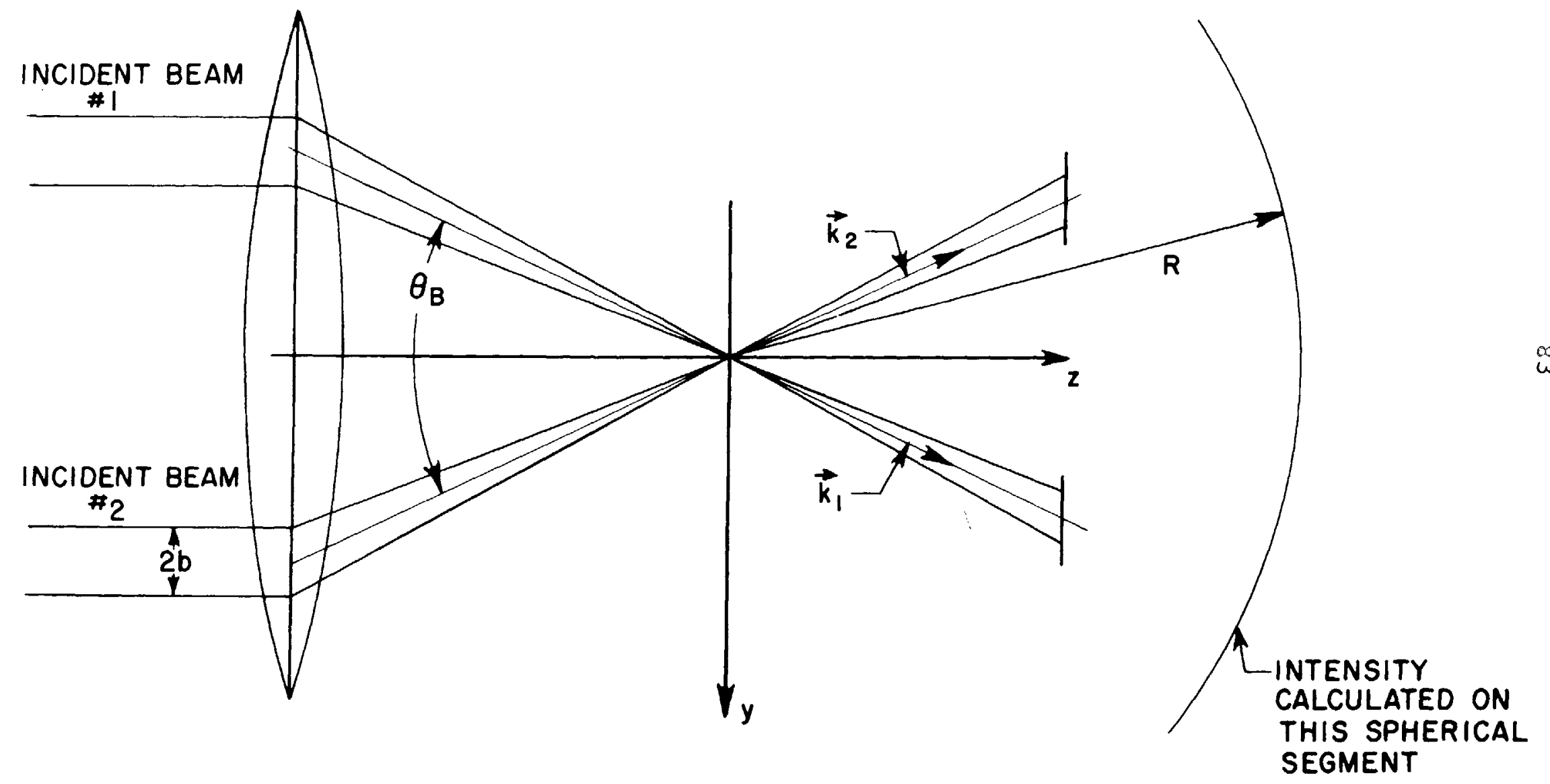

Fig. 10. Dual-Scatter system 


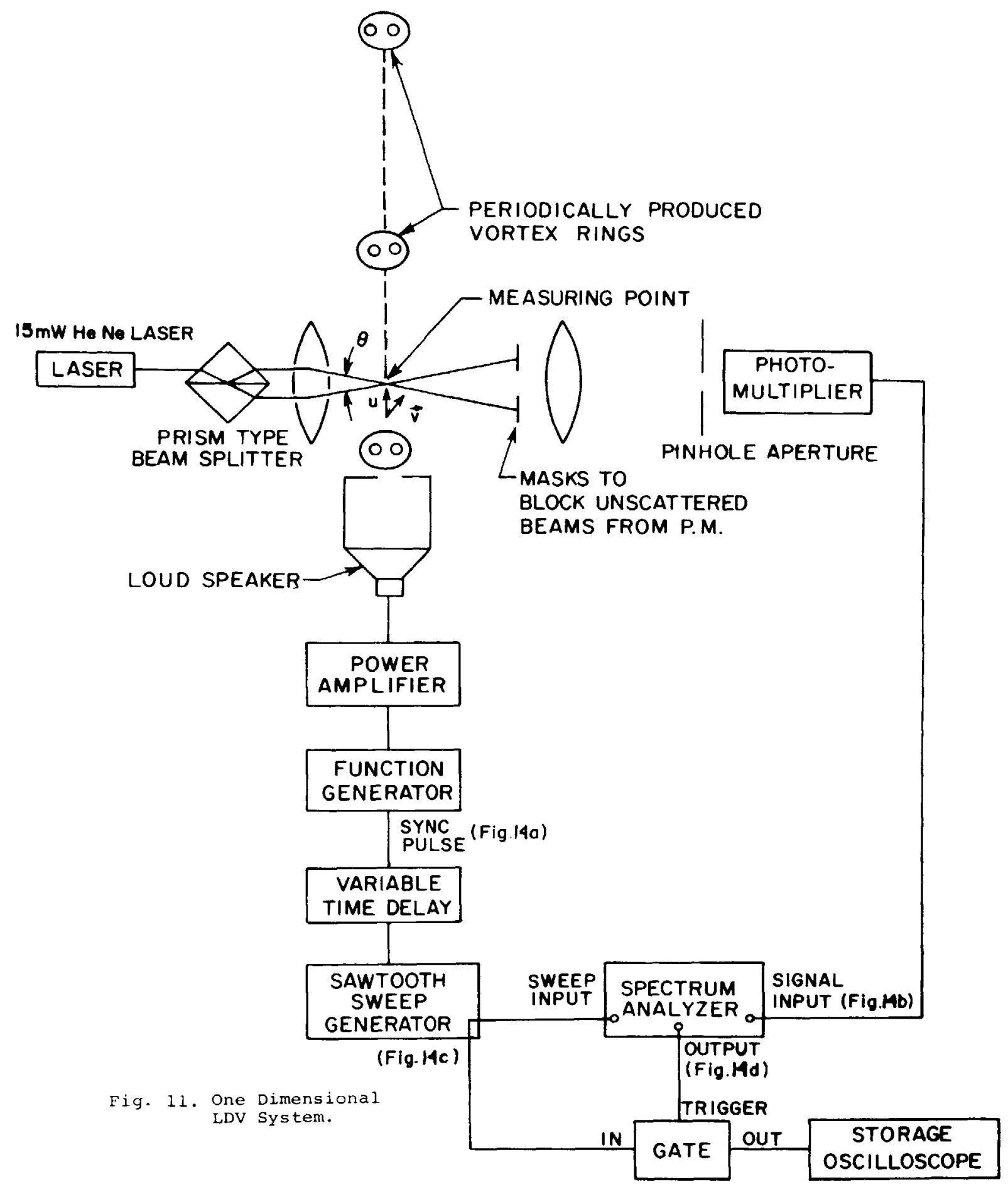




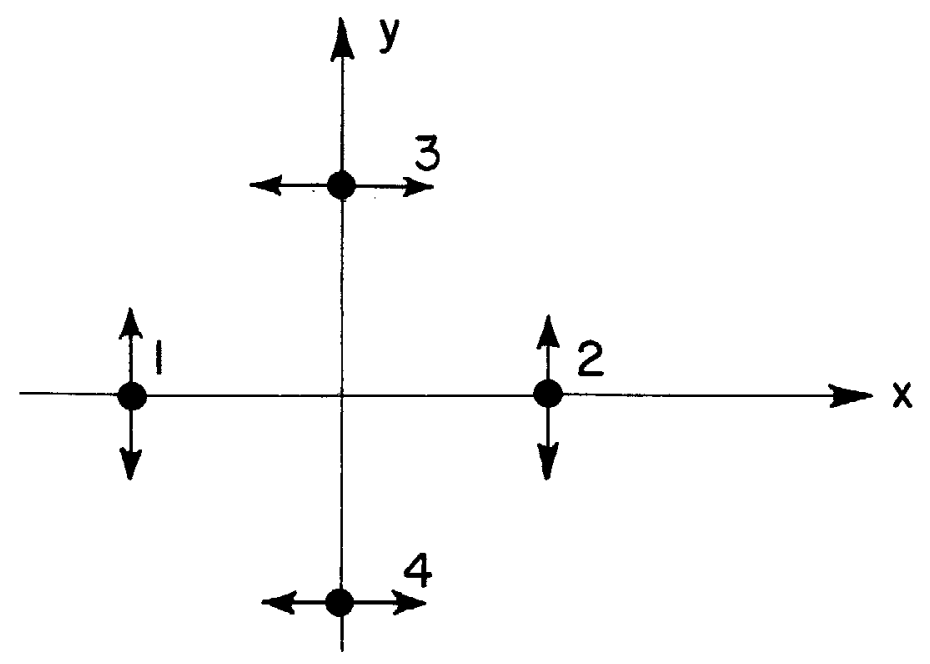

Fig. 12. Beam pattern for two dimensional LDV looking toward laser. The arrows indicate the direction of polarization. Beams 1 and 2 measure velocity component in the $x$ direction and beams 3 and 4 in the $y$ direction. 


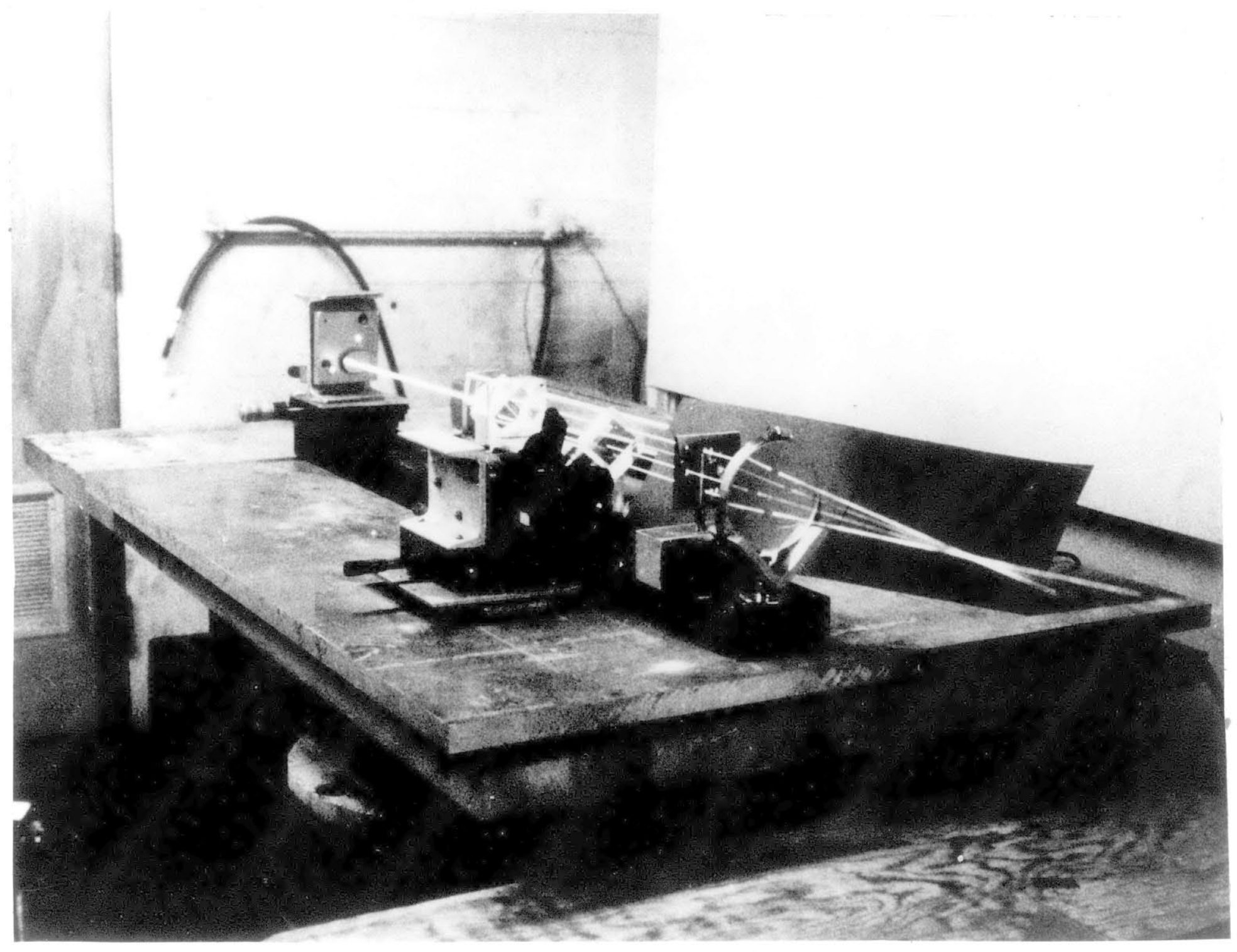

Fig. 13. Two-dimensional LDV system. 


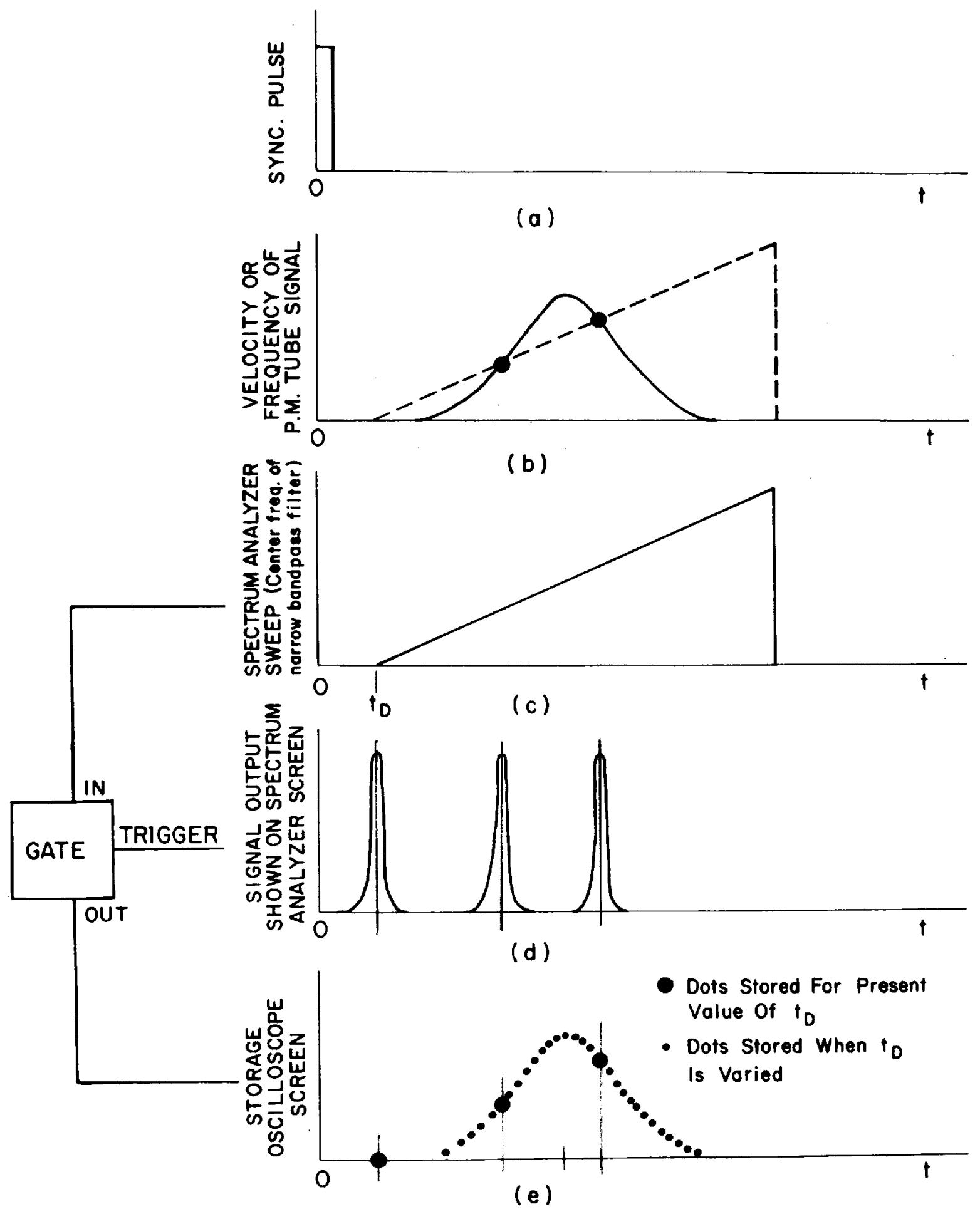

Fig. 14. Time diagrams for electronic system. 


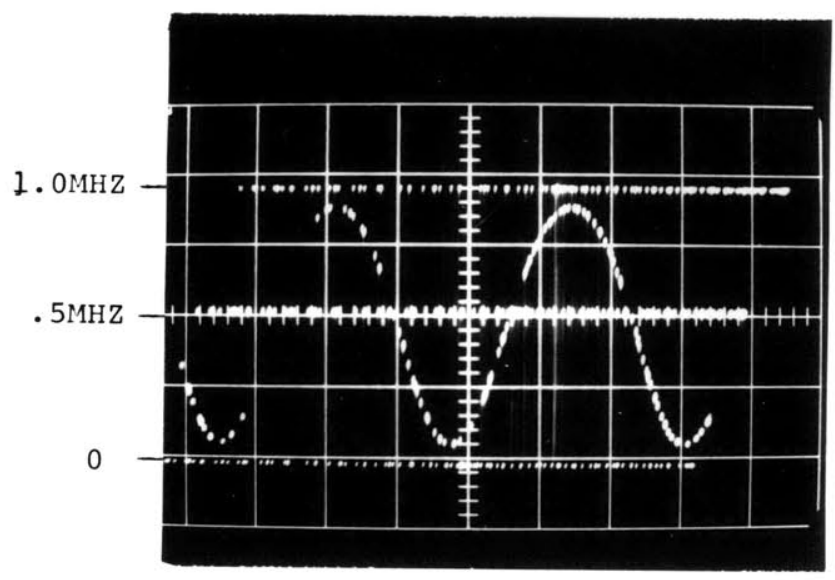

Fig. 15. Demodulation of Test Signal

$$
\begin{aligned}
& f_{O}=500 \mathrm{kHz} \\
& D f=450 \mathrm{kHz} \\
& f_{M}=33 \mathrm{cy} / \mathrm{sec}
\end{aligned}
$$




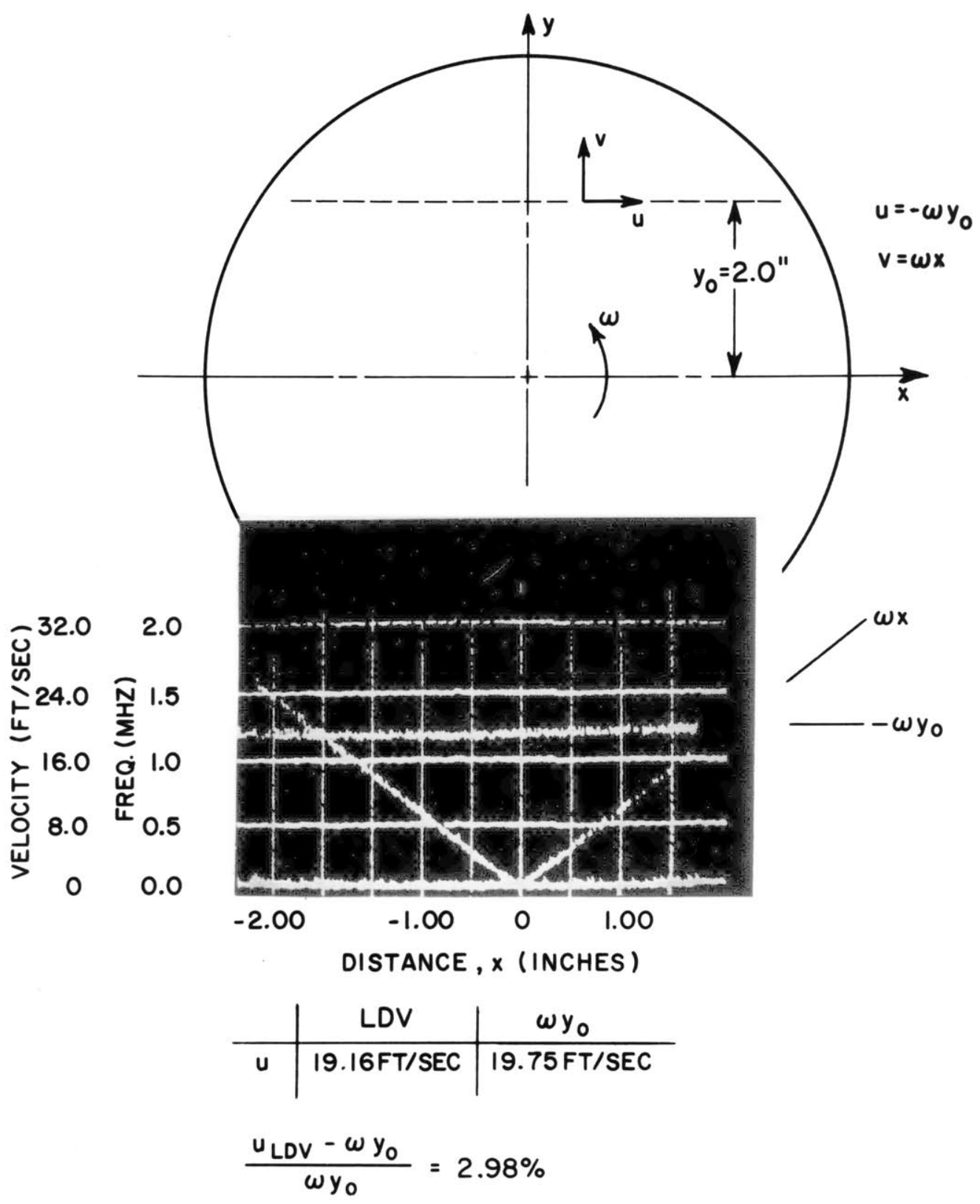

Fig. 16. Linearity and Velocity Check Using Spinning Disk 


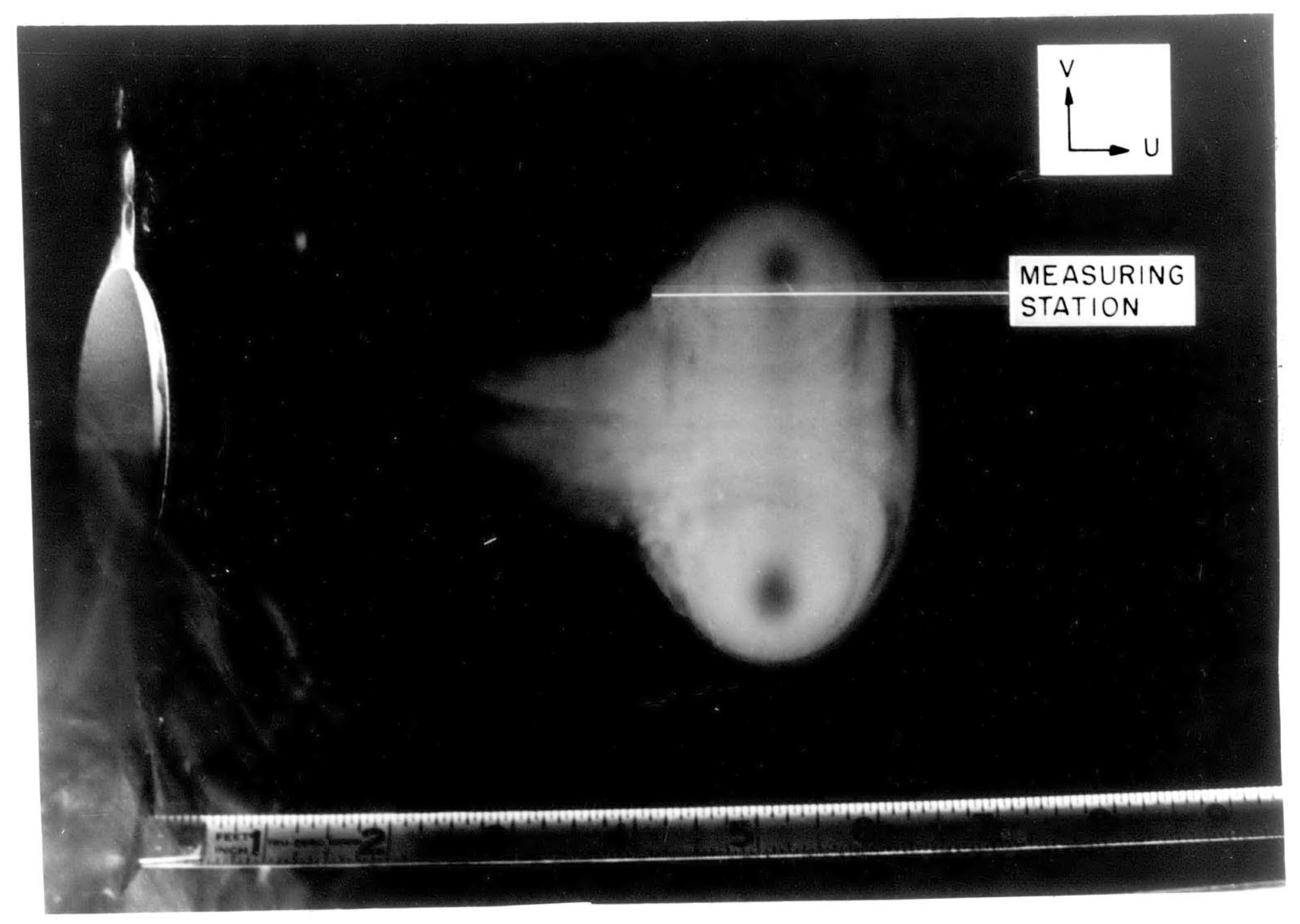

Fig. 17. Vortex ring. 


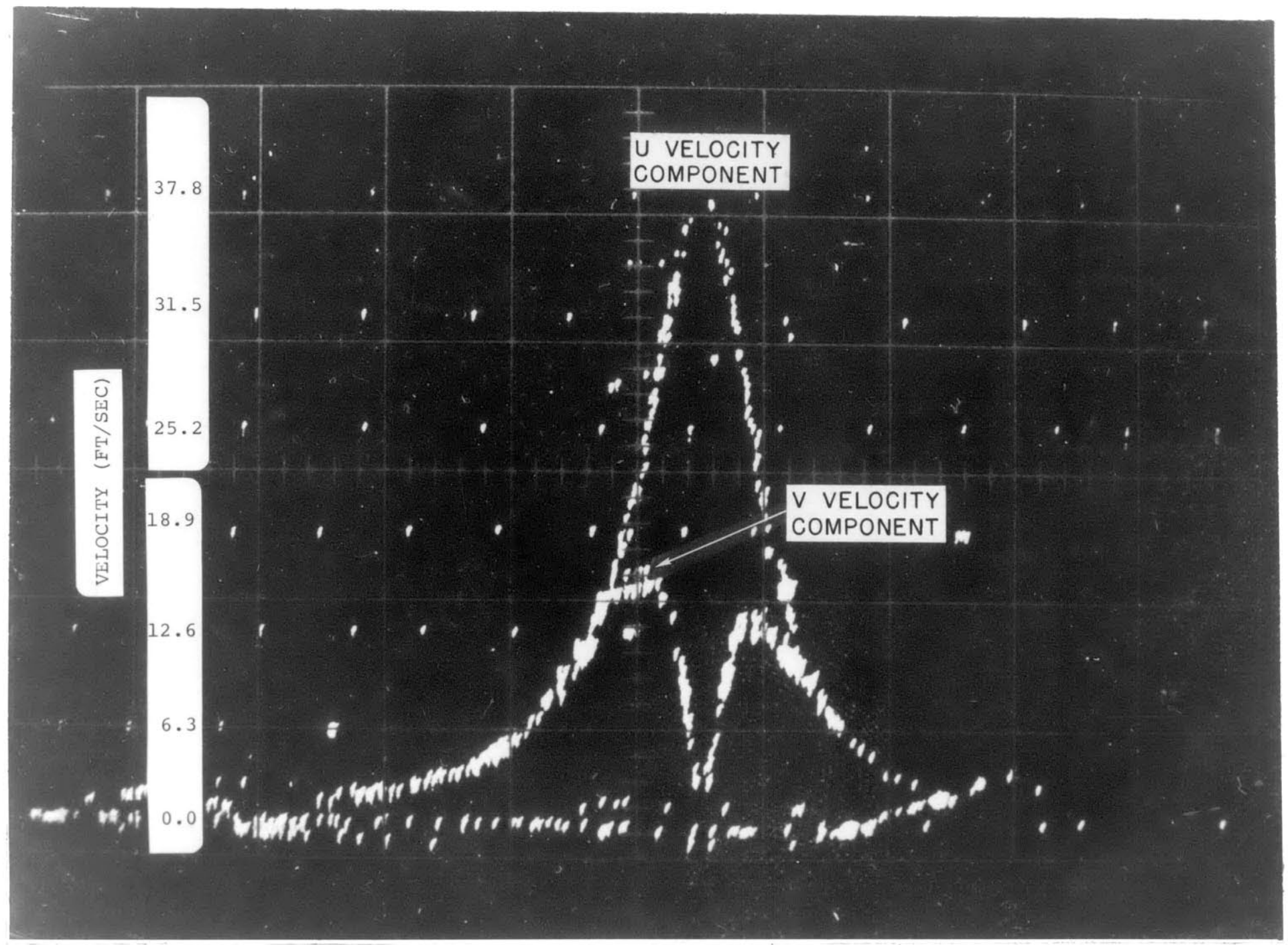

Fig. 18. Velocity distribution through vortex ring. Time Scale: $5 \mathrm{~ms} / \mathrm{cm}$. 


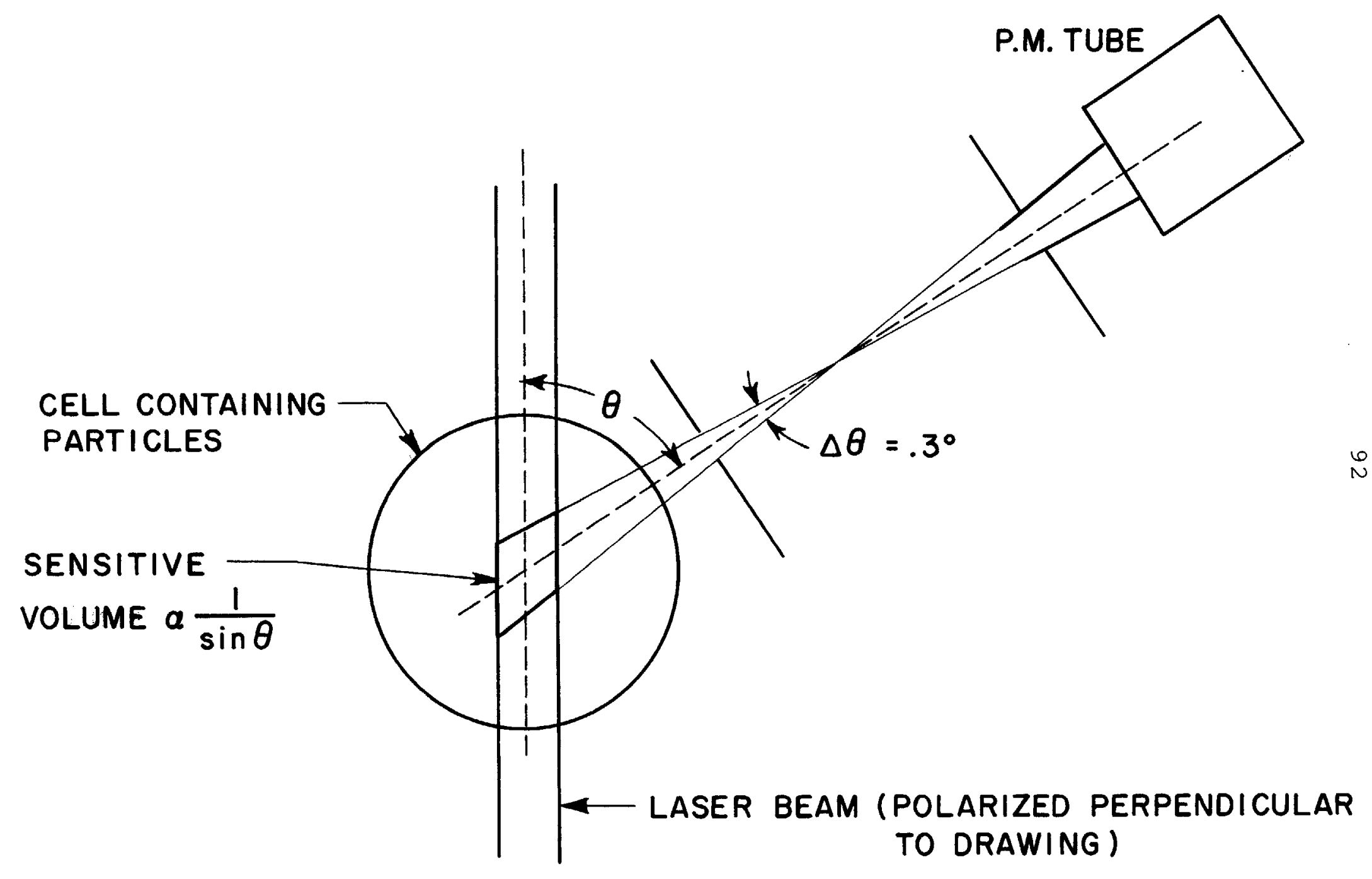

Fig. 19. Setup for Measuring Scattering Intensity Distribution $i_{\perp}$ 


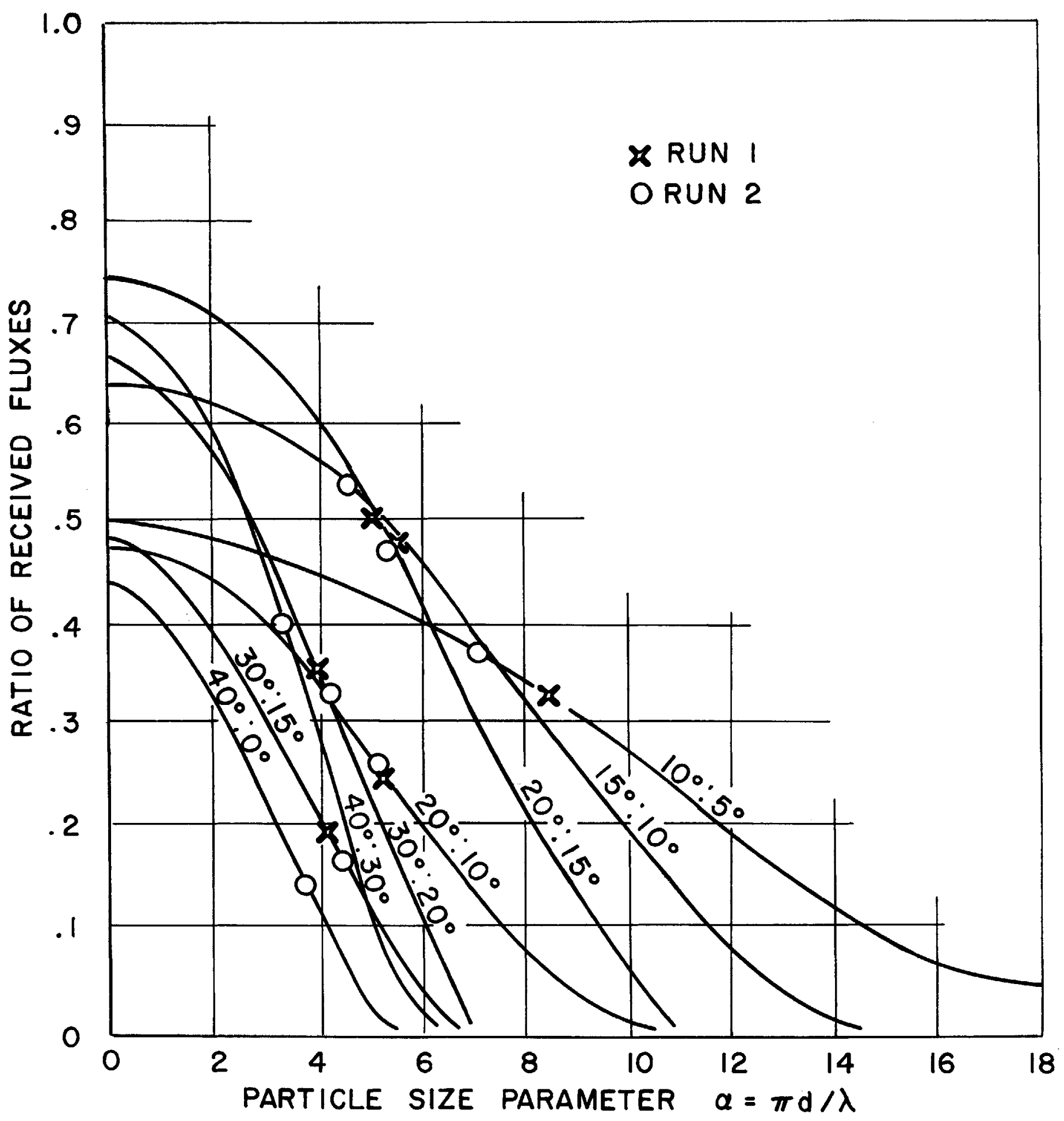

Fig. 20. Scheme of Particle Sizing Based on Scattering Measurements Within the Forward Lobe. The Solid Curves are Ratios of $i_{\perp}$ versus $\alpha$. i.e. the $10^{\circ}: 5^{\circ}$
is $\frac{i_{\perp}\left(10^{\circ}\right)}{i_{\perp}\left(15^{\circ}\right)}$ vs. $\alpha$. 


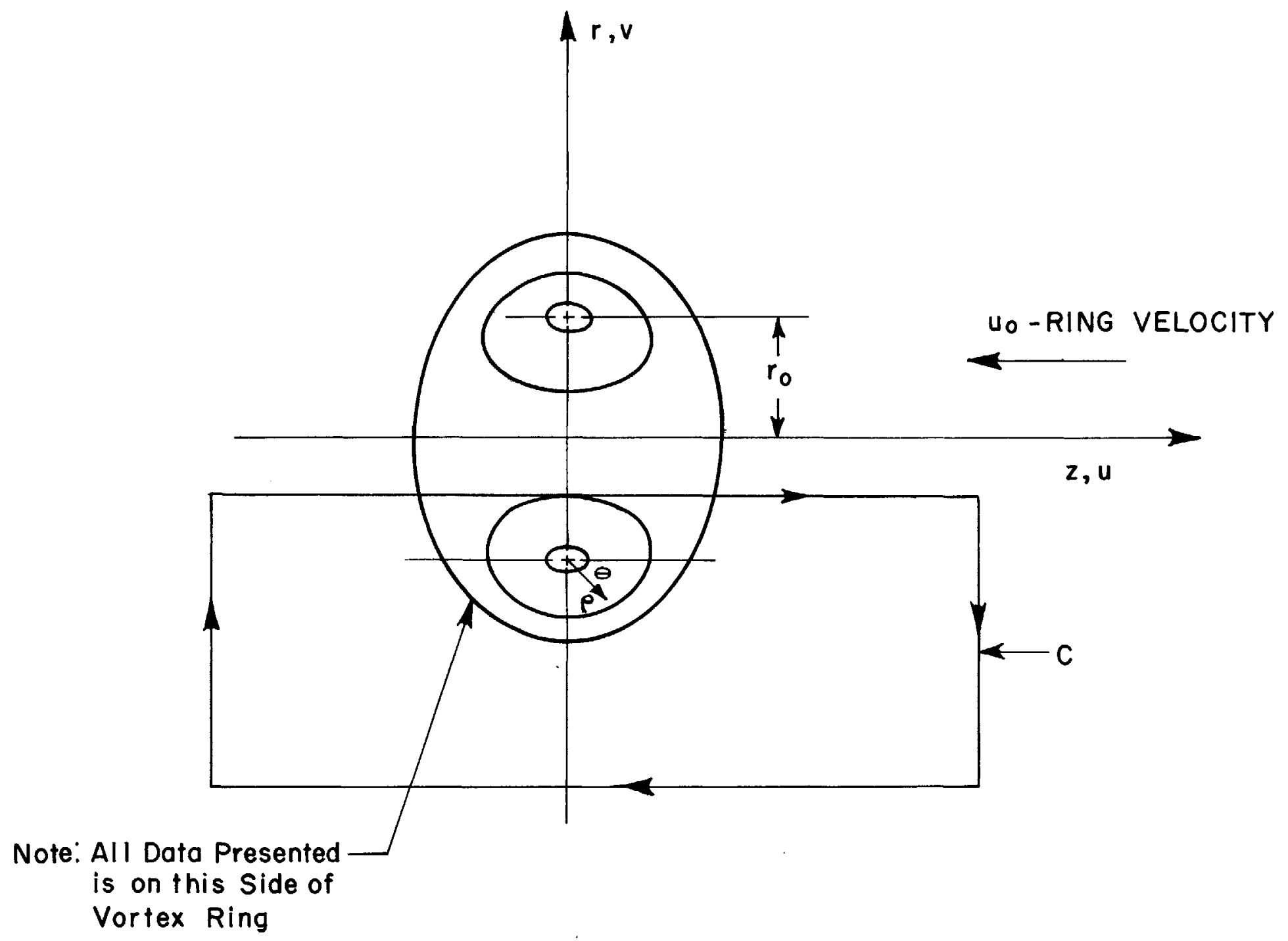

Fig. 21. Coordinate system for vortex ring data analysis. 


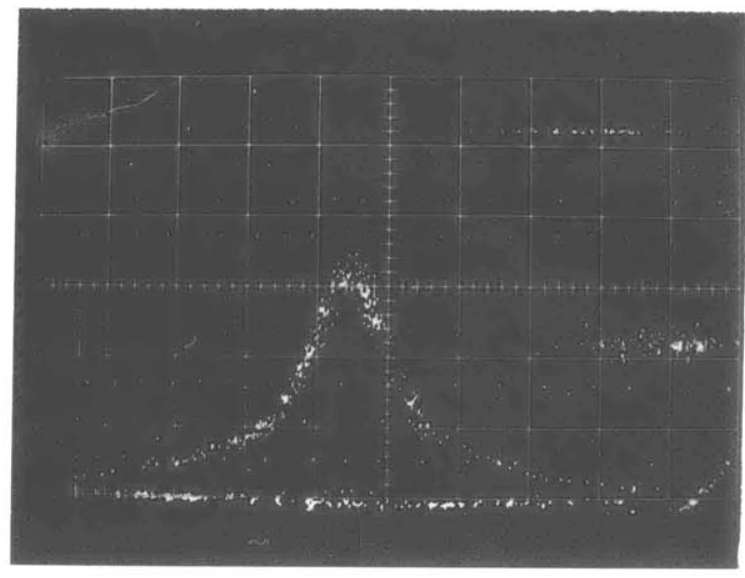

Fig. 22a. U component of velocity at $r / r_{0}=-.876$ for

Ring No. 1.

Time Scale $20 \mathrm{MS} / \mathrm{cm}$

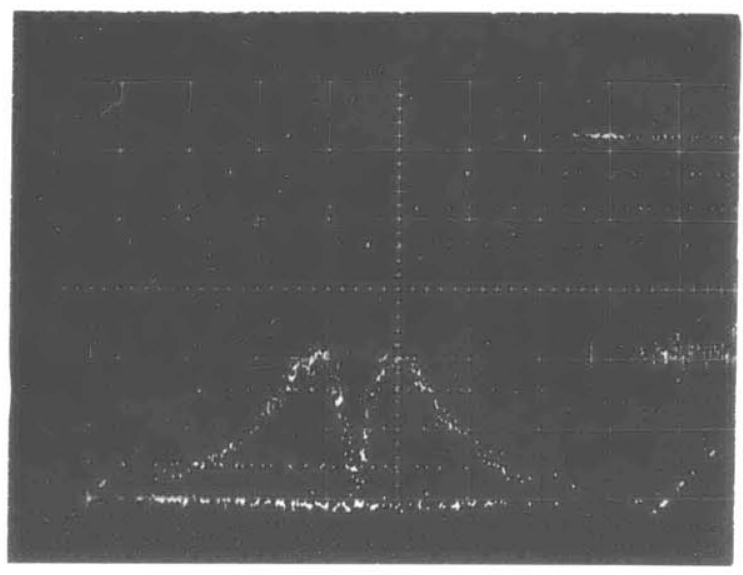

Fig. 22 b. V component of velocity at $r / r=-1.08$ for Ring N8. 1.

Time Scale $20 \mathrm{MS} / \mathrm{cm}$ 


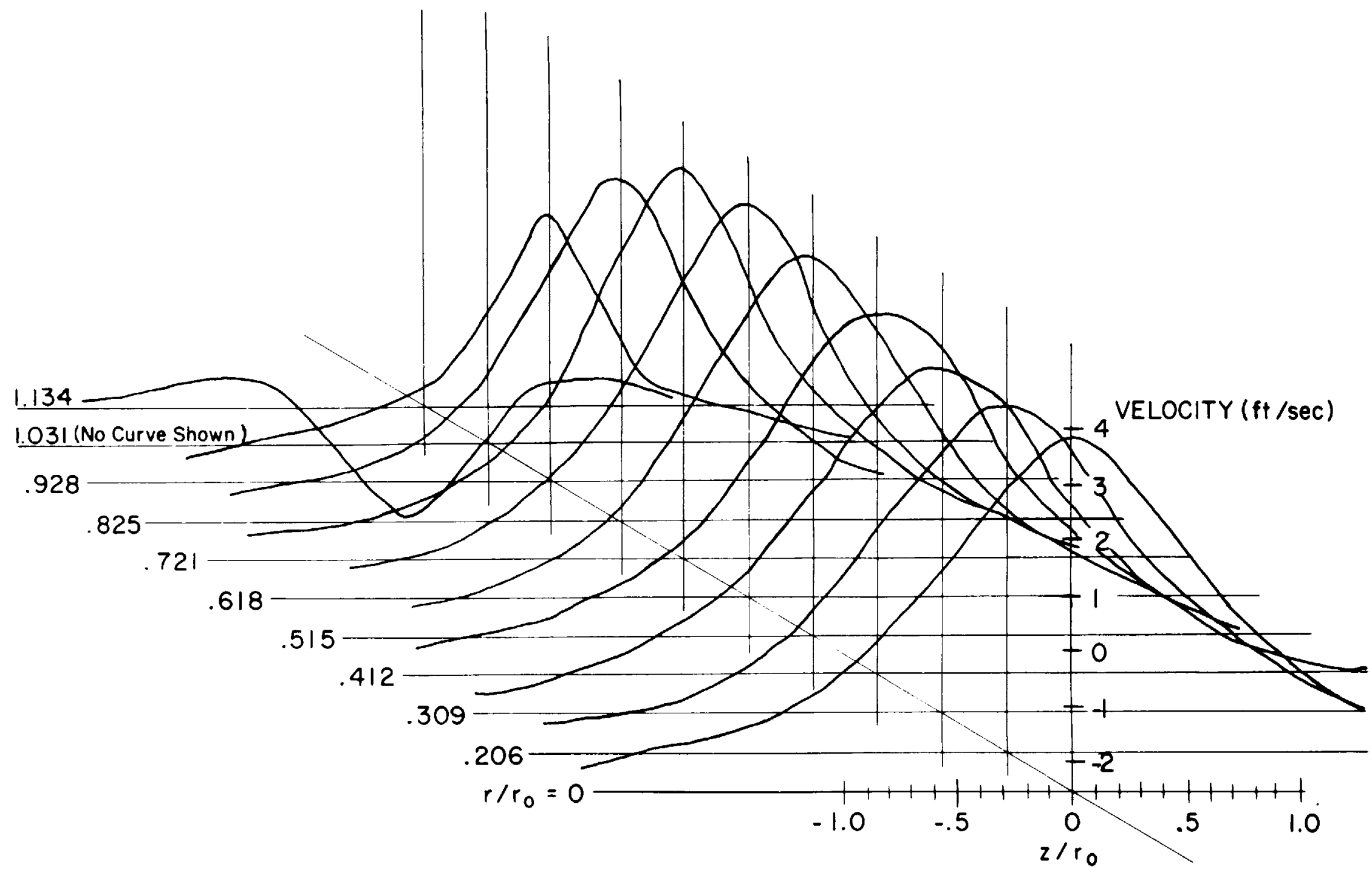

$\stackrel{6}{\sigma}$

Fig. 23. U component of velocity versus axial distance at various radial stations of Ring No. 1 . 


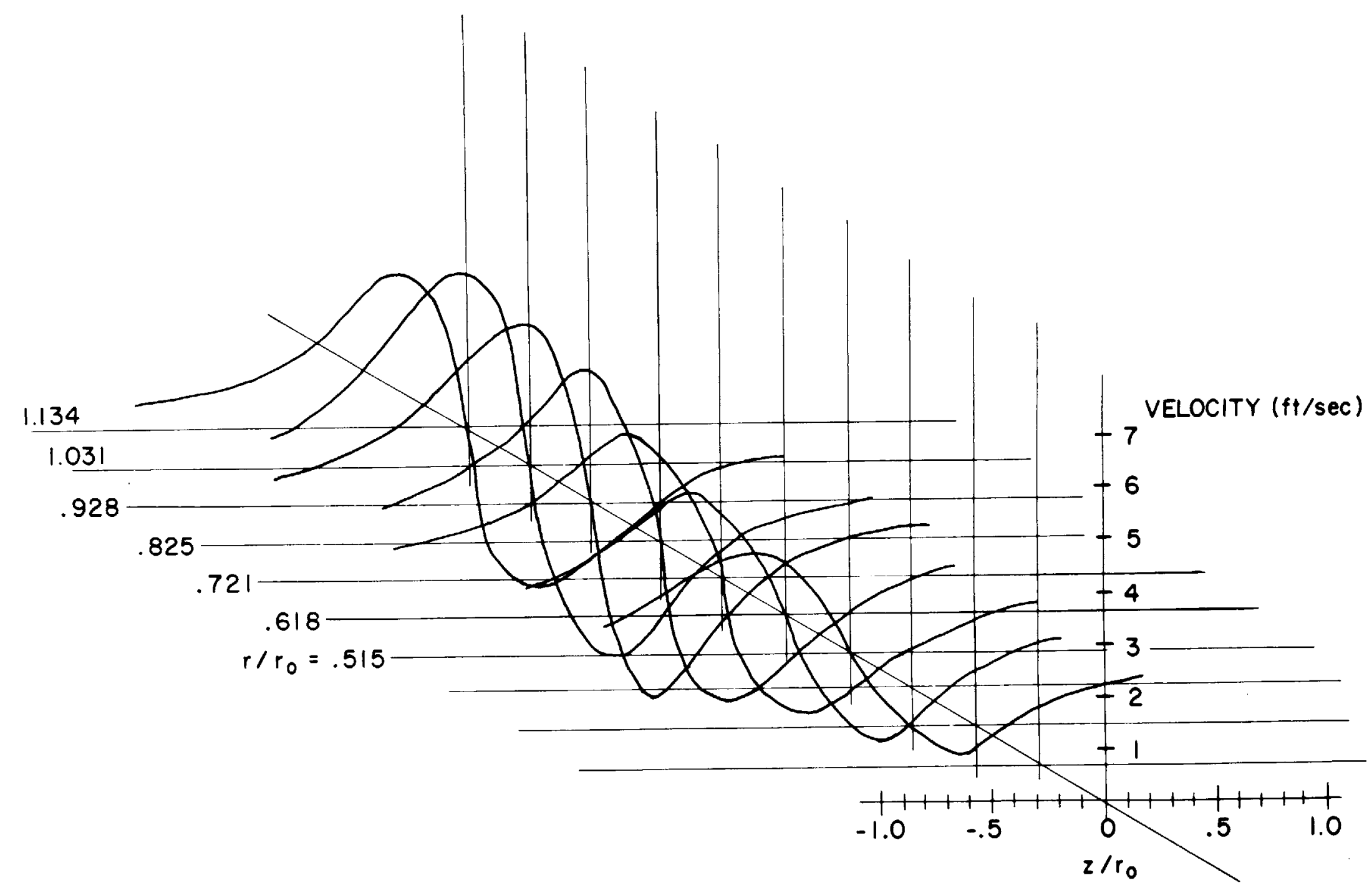

Fig. 24, V component versus axial distance at various radial stations of Ring No. 1 


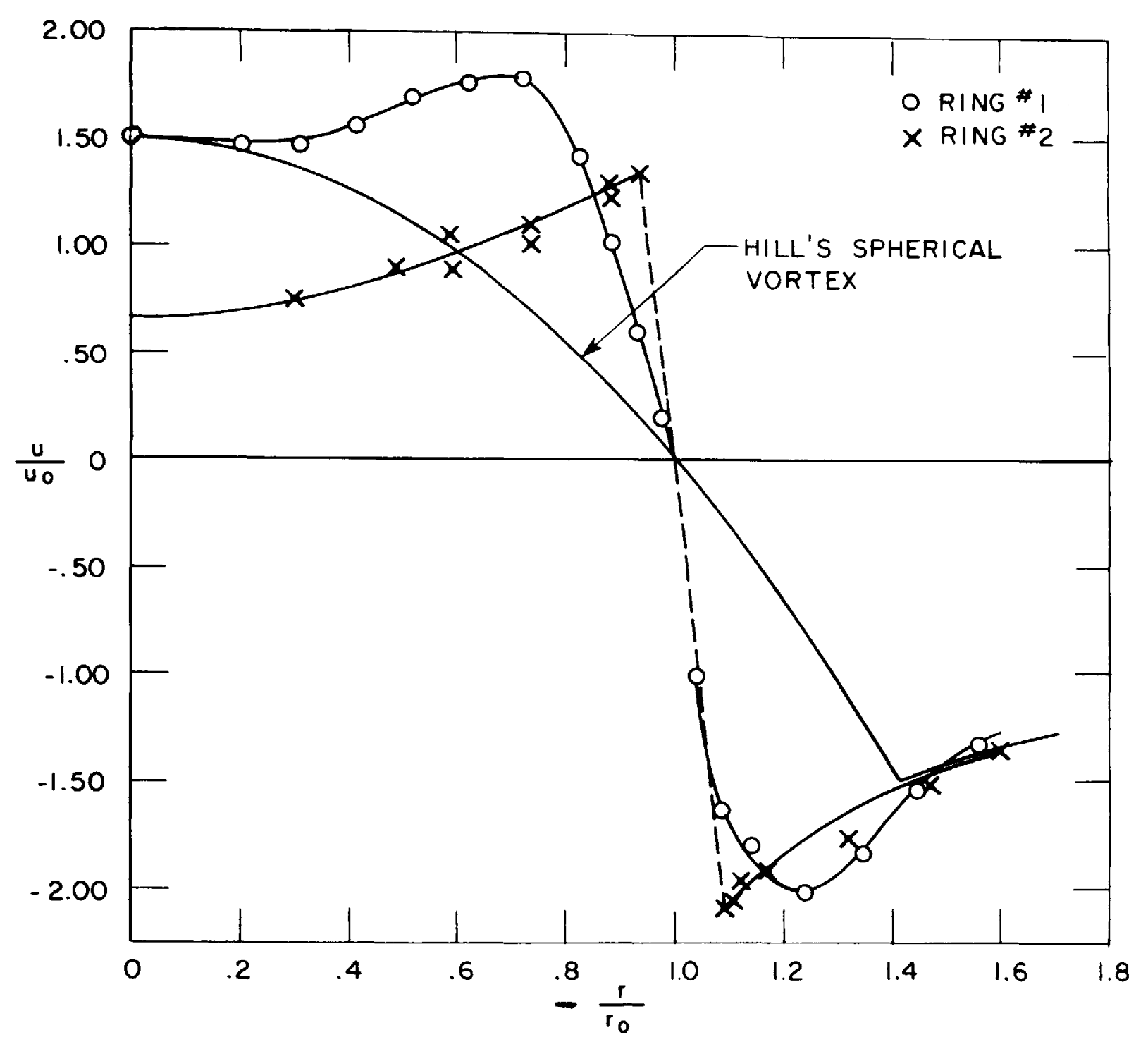

Fig. 25. $\mathrm{U} / \mathrm{u}_{\mathrm{O}}$ versus $\mathrm{r} / \mathrm{r}_{\mathrm{O}}$ along $\mathrm{z} / \mathrm{r}_{\mathrm{O}}=0$. 


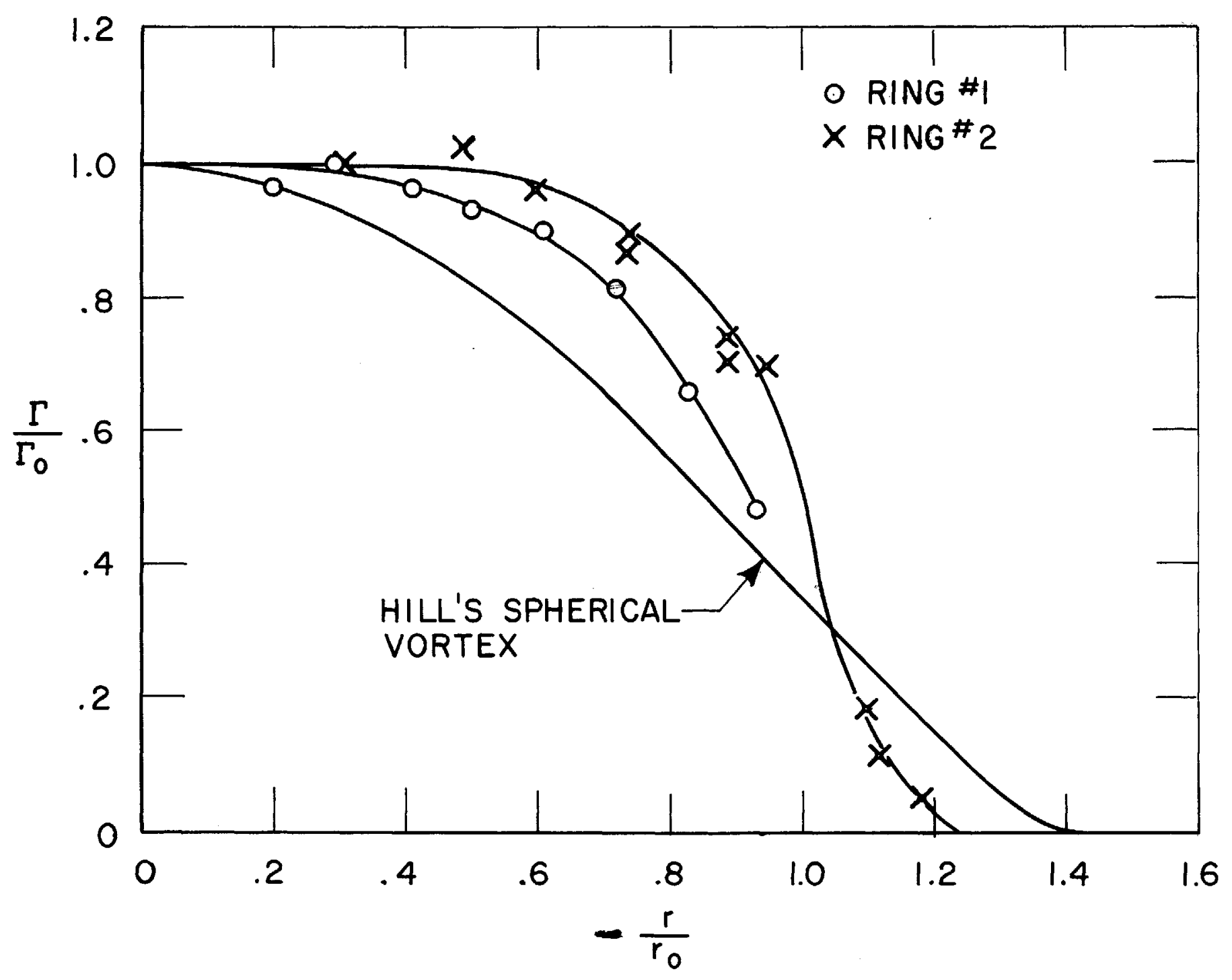

Fig. 26. $\Gamma / \Gamma_{0}$ versus $r / r_{0}$ 


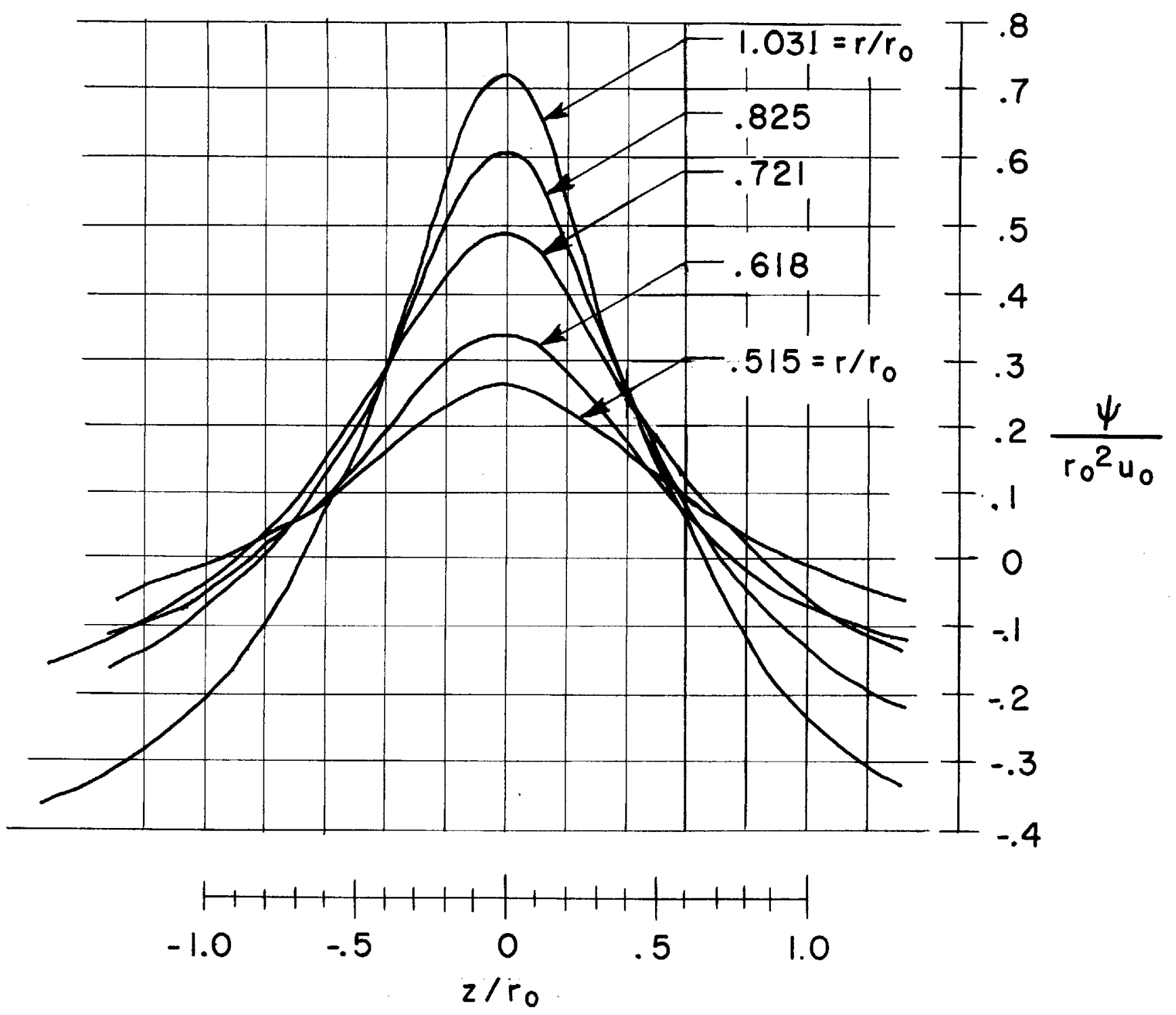

Fig. 27. $\psi / \mathrm{r}_{0}^{2} U_{0}$ versus $\mathrm{z} / \mathrm{r}_{0}$ at various radial stations 


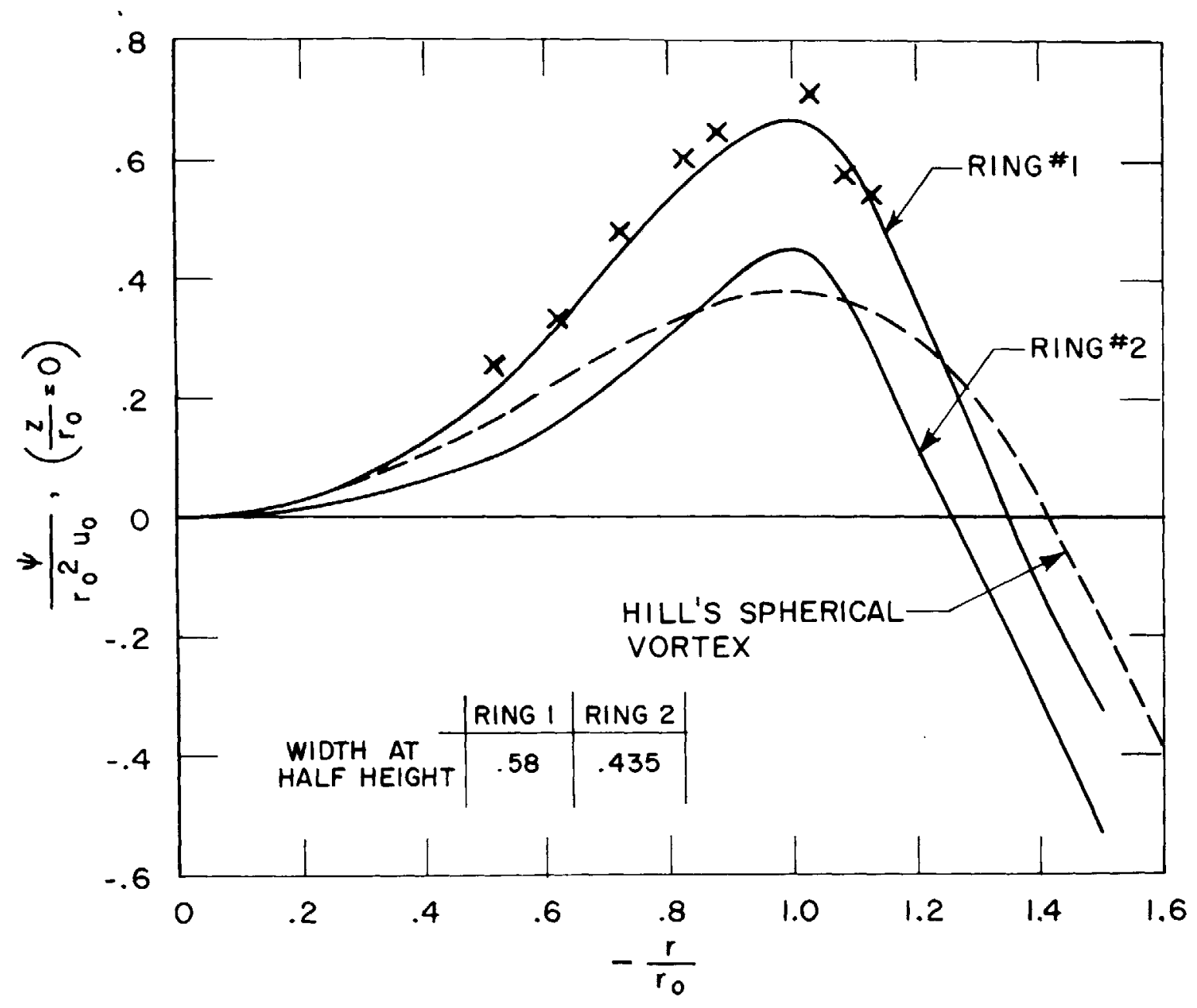

Fig. 28. $\psi / r_{0}{ }^{2} U_{0}$ versus $r / r_{0}$ at $z / r_{0}=0$.

X - Calculated from Eq. 2 .

Solid curves calculated from Eq. 3. 


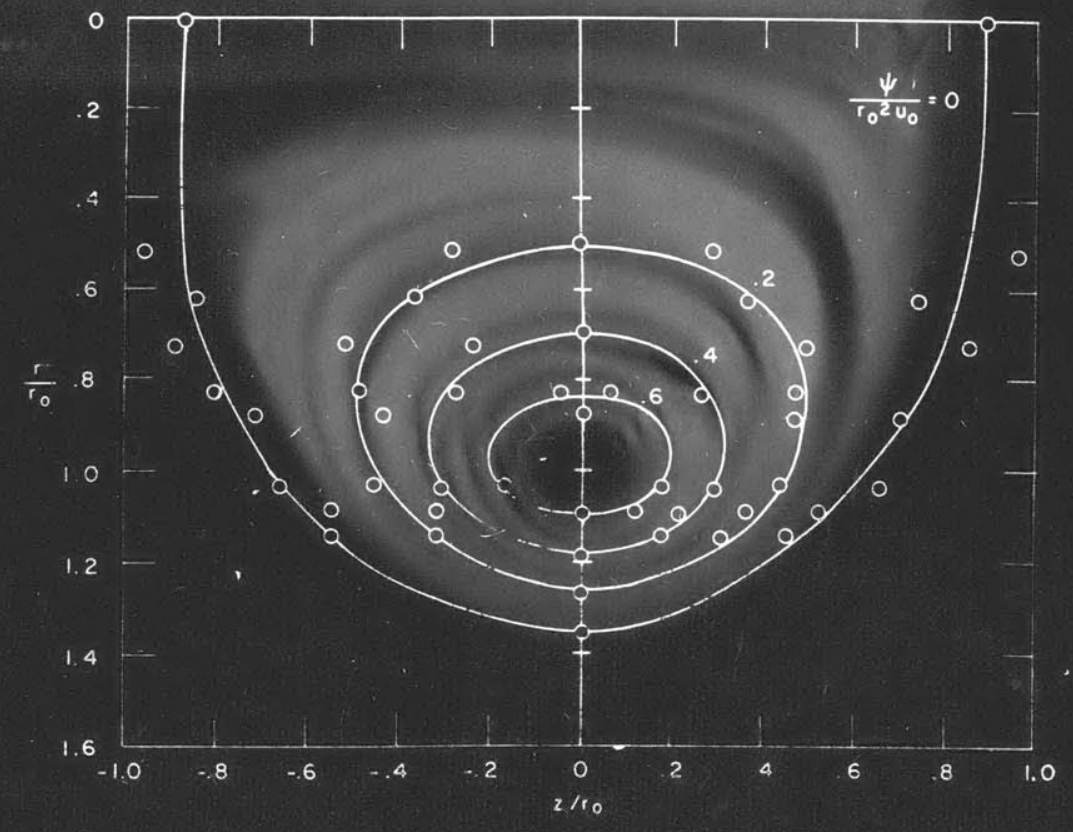

Fig. 29. Side view of ring No. 1 with measured stream lines superposed. 


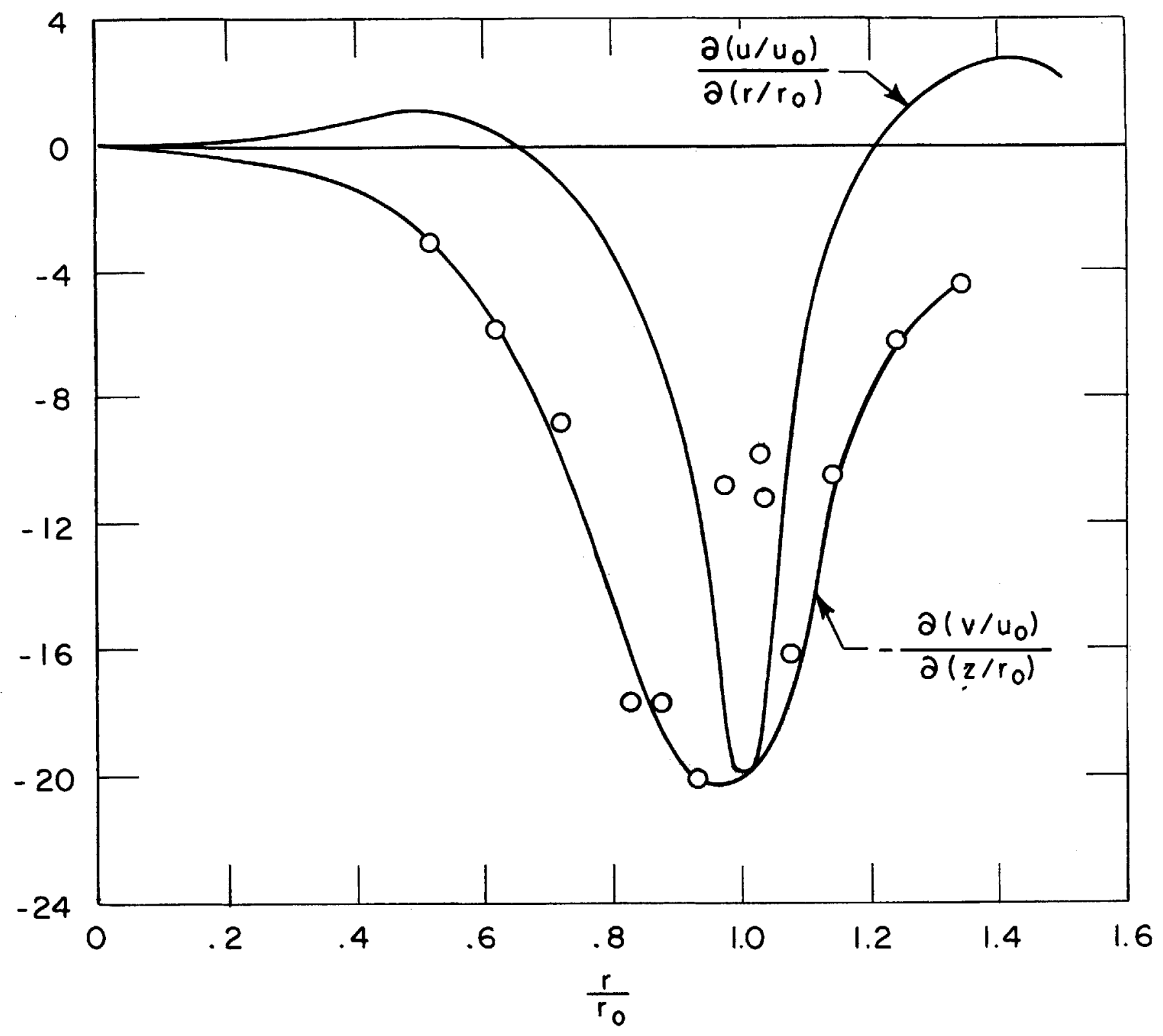

Fig. 30. Partial Derivatives Used For Calculating the Vorticity. 


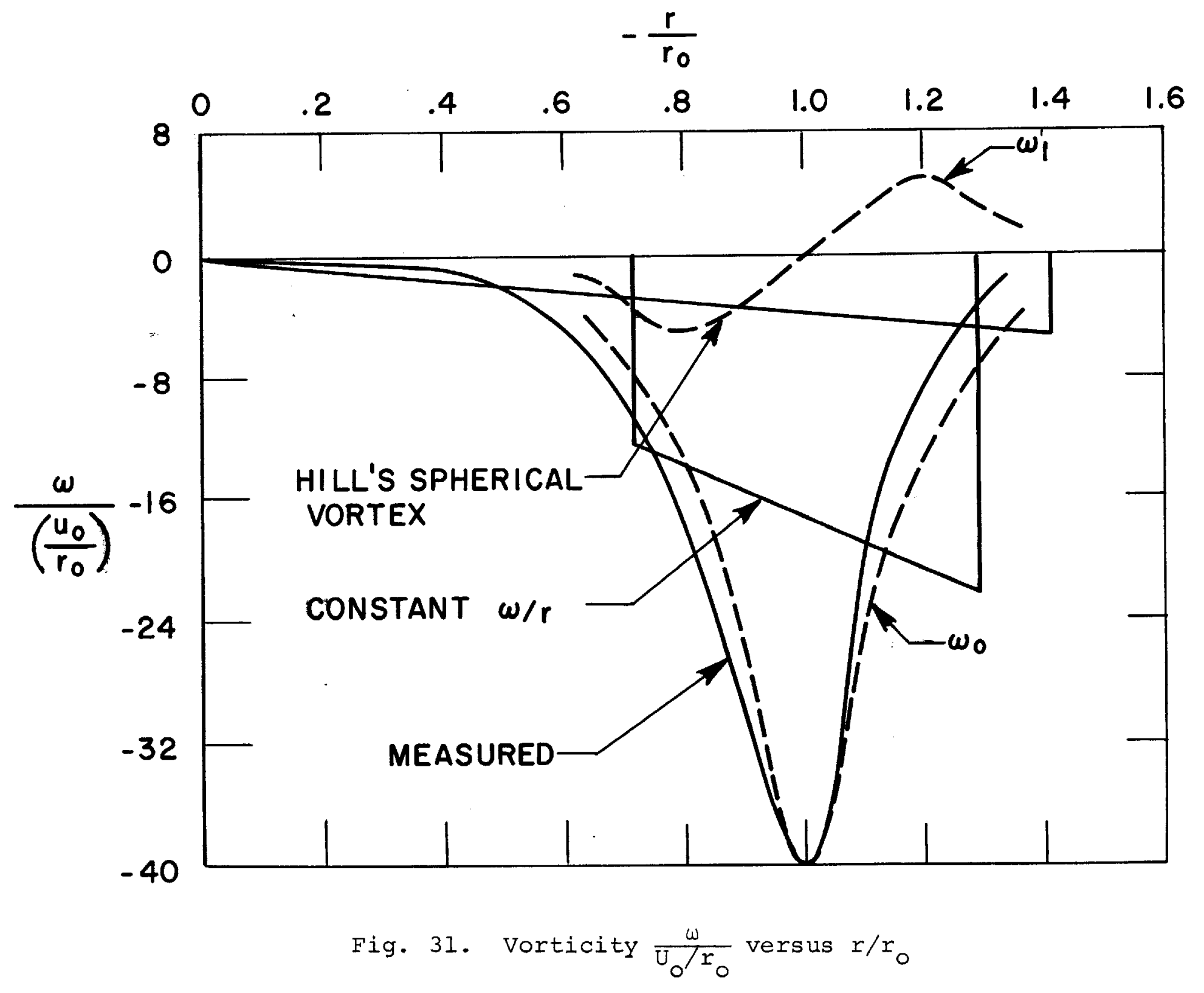




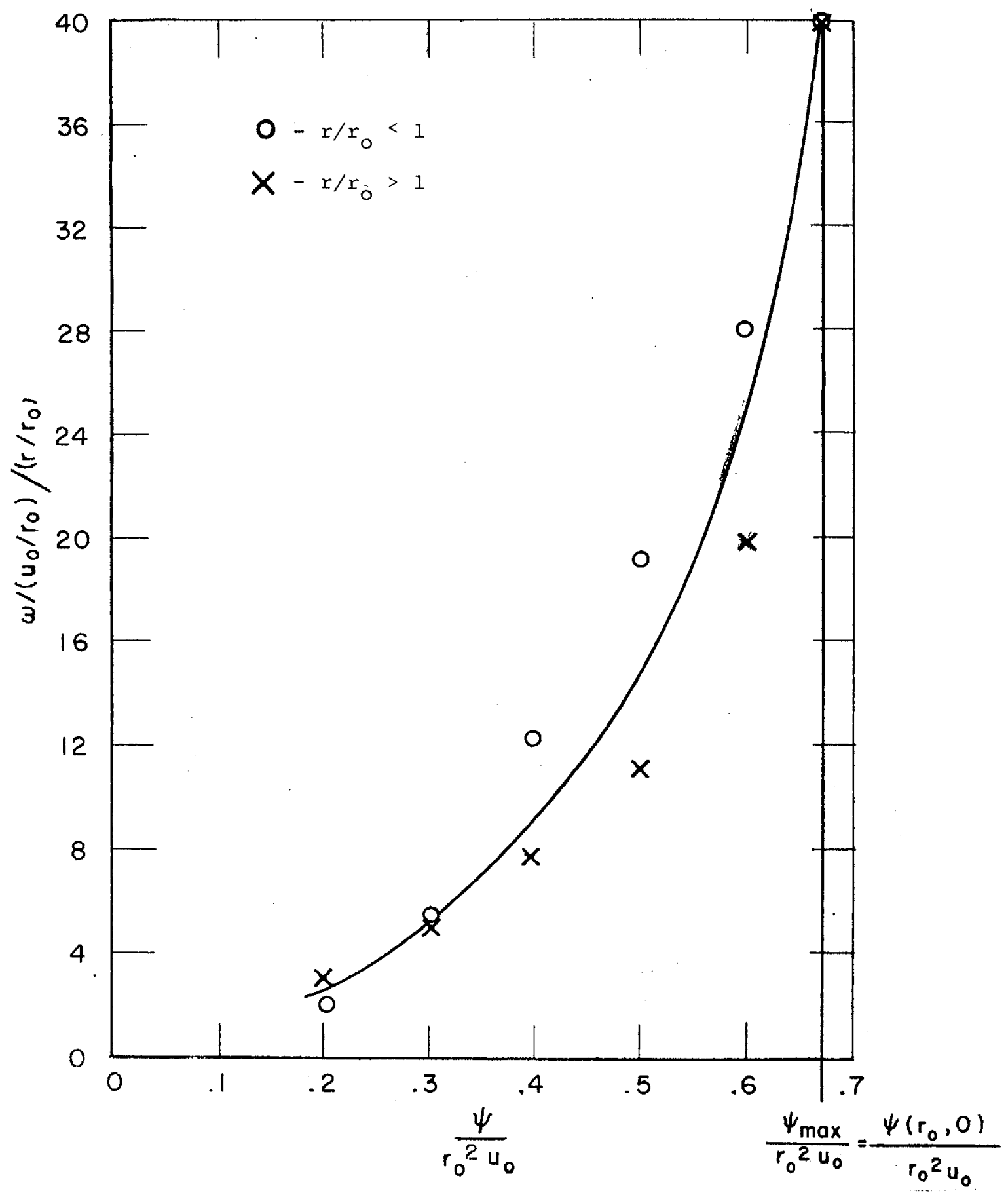

Fig. 32. Non-dimensional $\omega / \mathbf{r}$ versus $\psi$. 


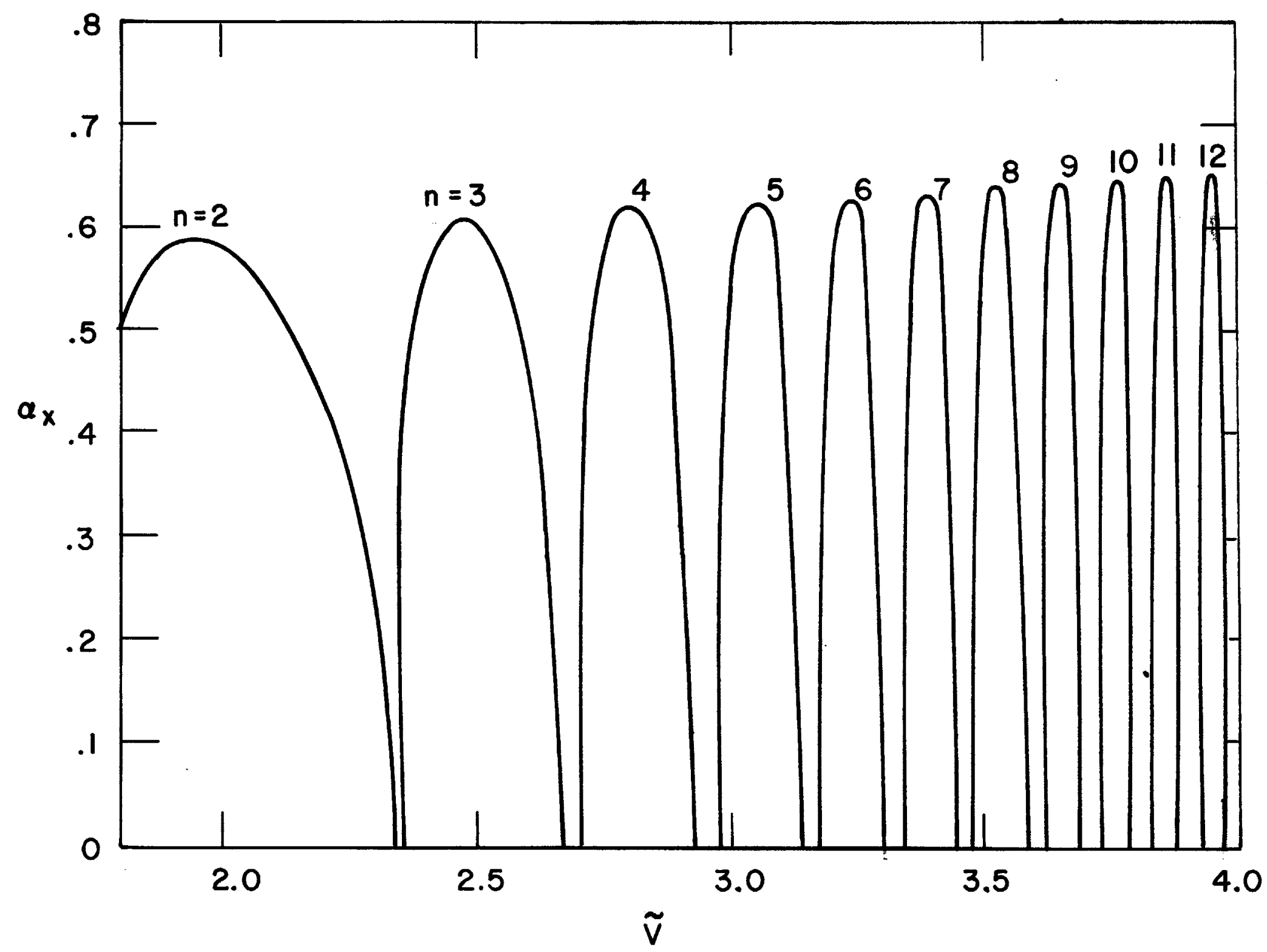

홍

Fig. 33. Nondimensional Spatial Amplification Rate $\alpha_{x}$ of the Vortex Ring Instability as a Function of $\widetilde{V}$ and Mode Number $n$. 


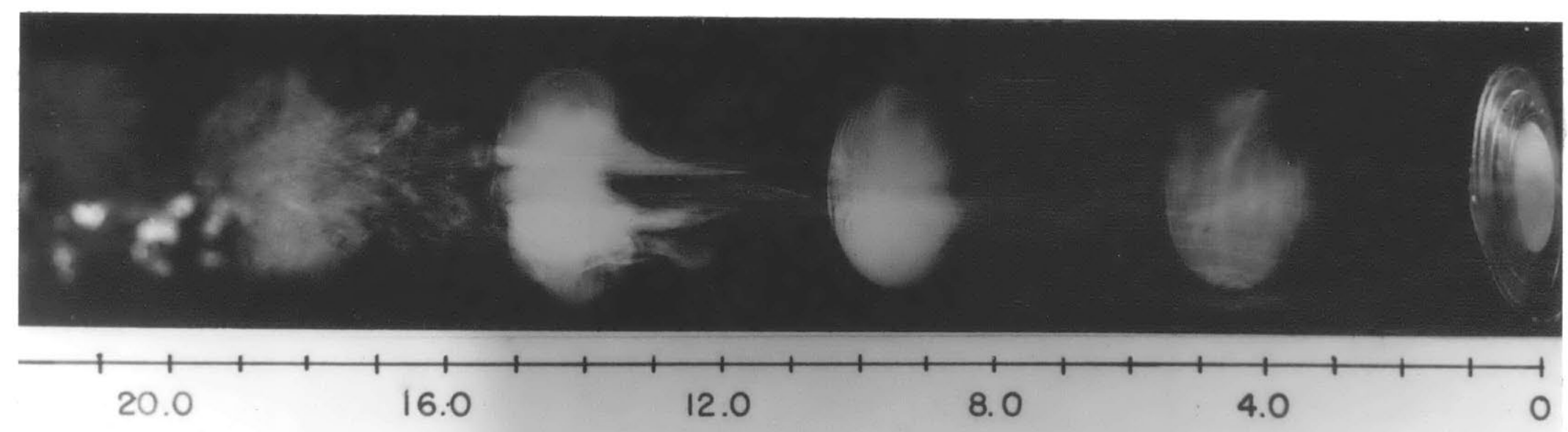

Fig. 34. Multiple flash side view photograph of $\mathrm{n}=7$ mode instability. 


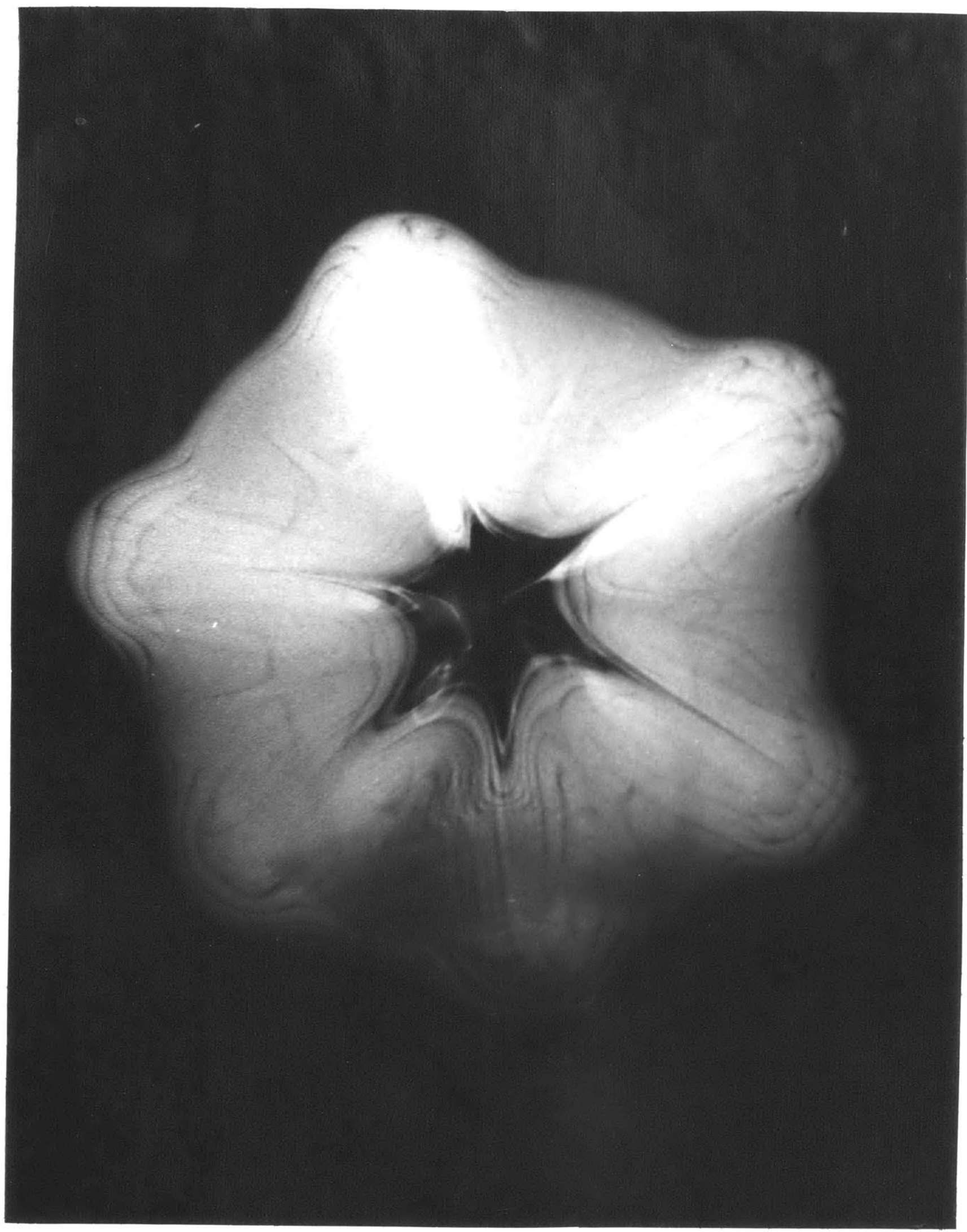

Fig. 35. Flow Visualization of the vortex ring instability $n=6$. 


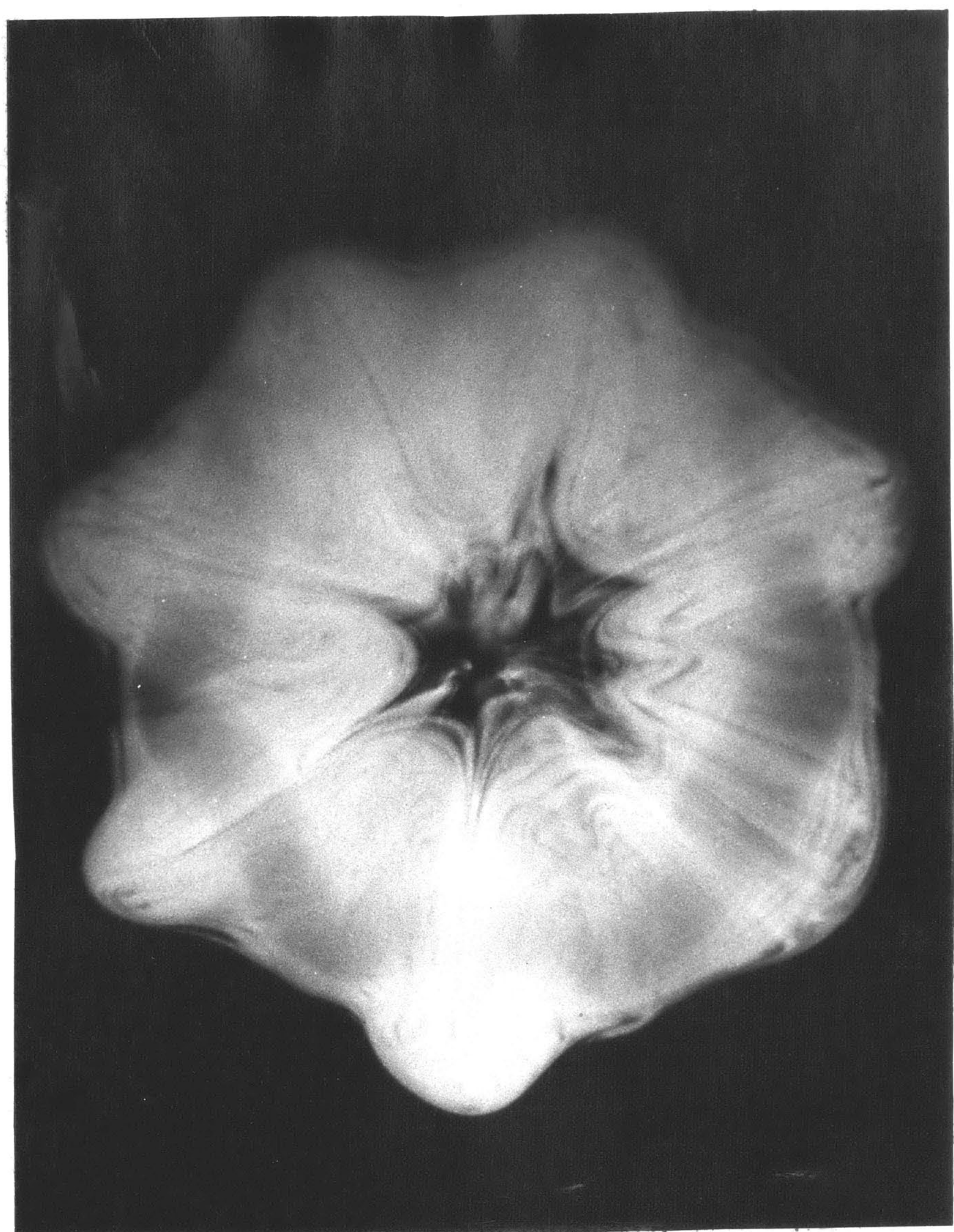

Fig. 36. Flow Visualization of the vortex ring instability $n=7$. 


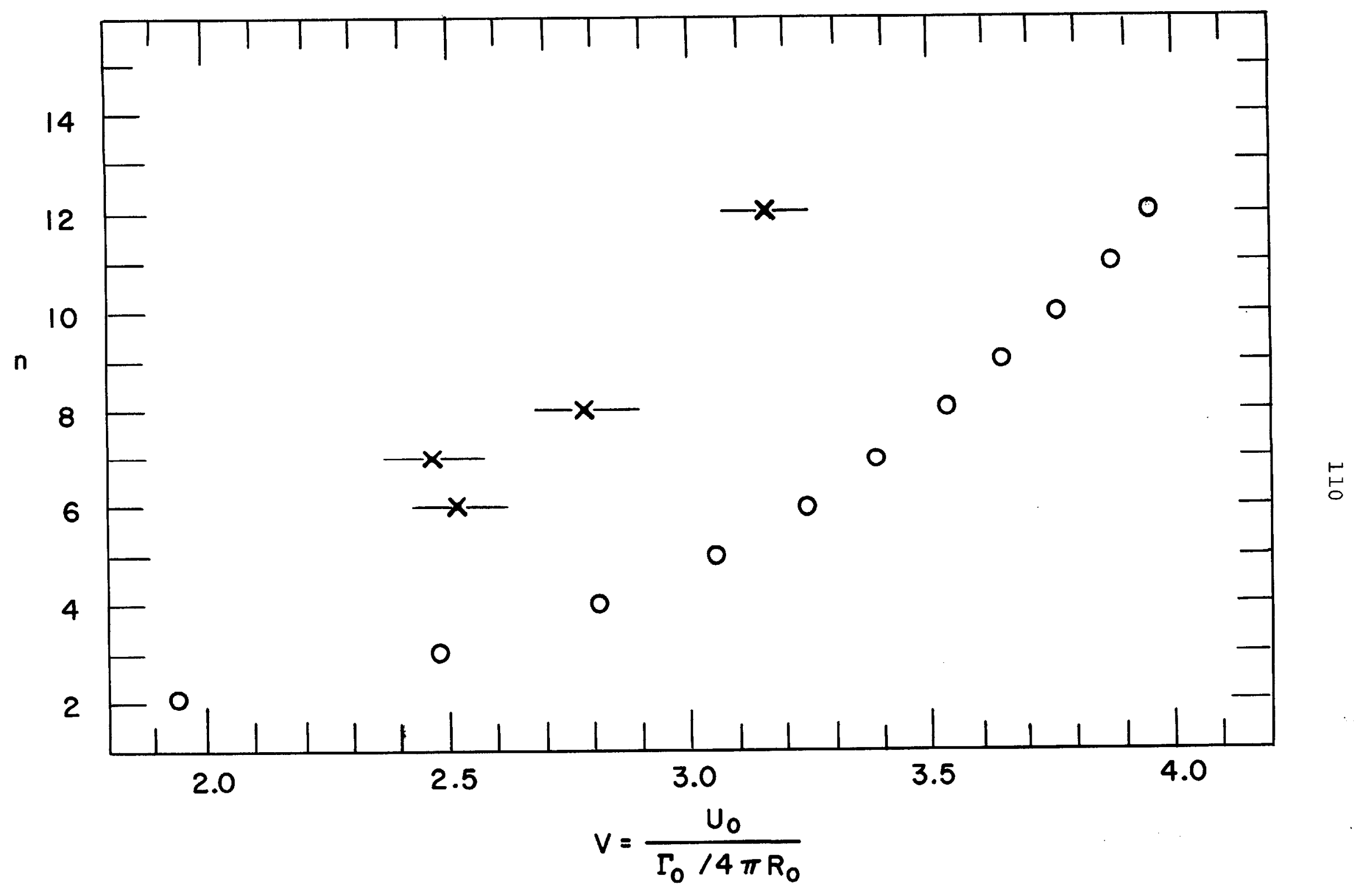

Fig. 37. Number of waves in the unstable mode as a function of $\widetilde{V}$ $\mathrm{X}$ experiment; $O$ theoretical prediction at maximum amplification. 


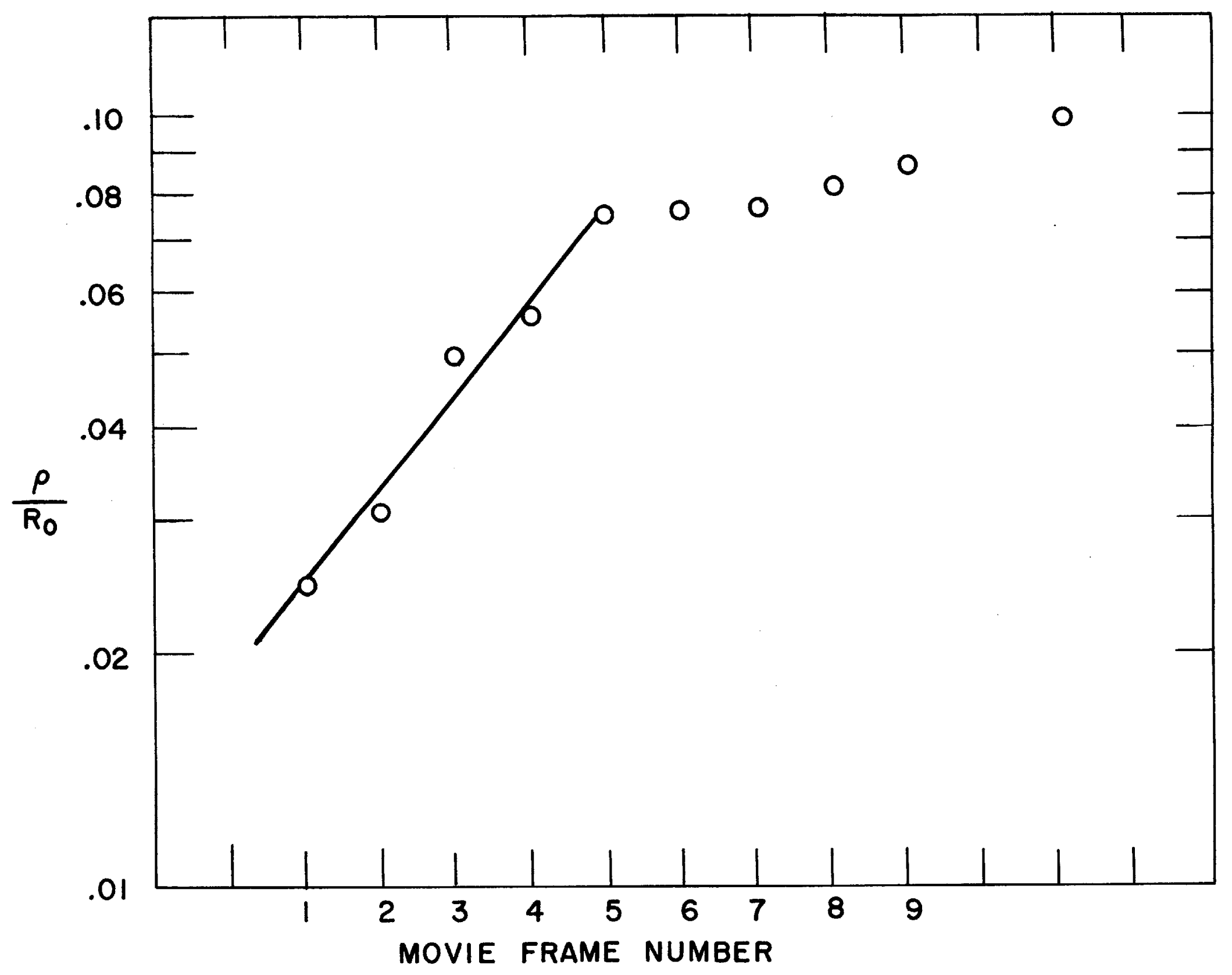

Fig. 38. Per cent radial perturbation for the vortex ring of Fig. 36 measured at each strobe movie frame (time between frames $14.5 \mathrm{msec}$ );--exponential growth $\alpha_{x}=.69$; theoretical prediction $\alpha_{x}=.64$. 


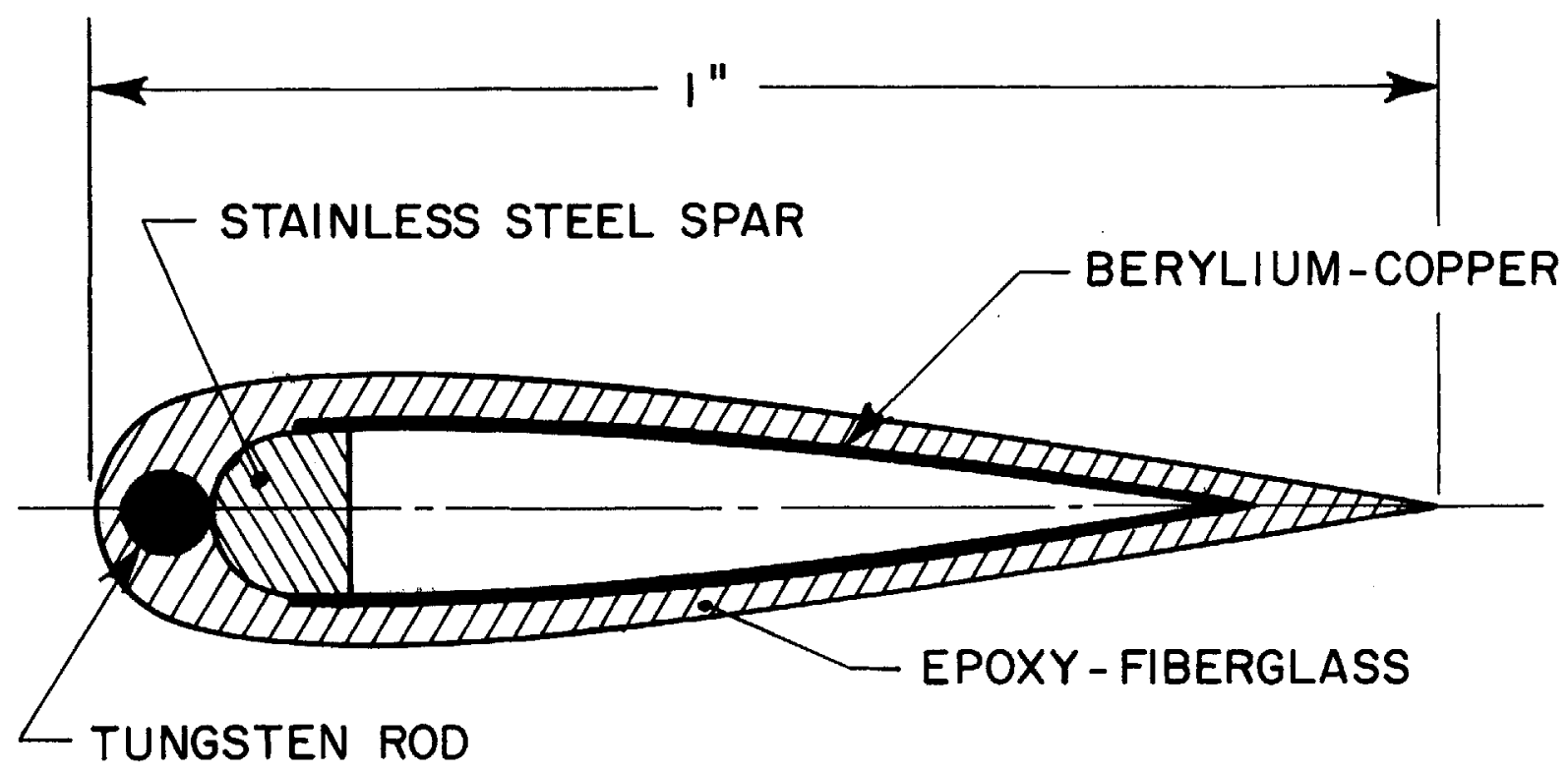

Fig. 39. Cross Section of Blade - NACA 0012. 


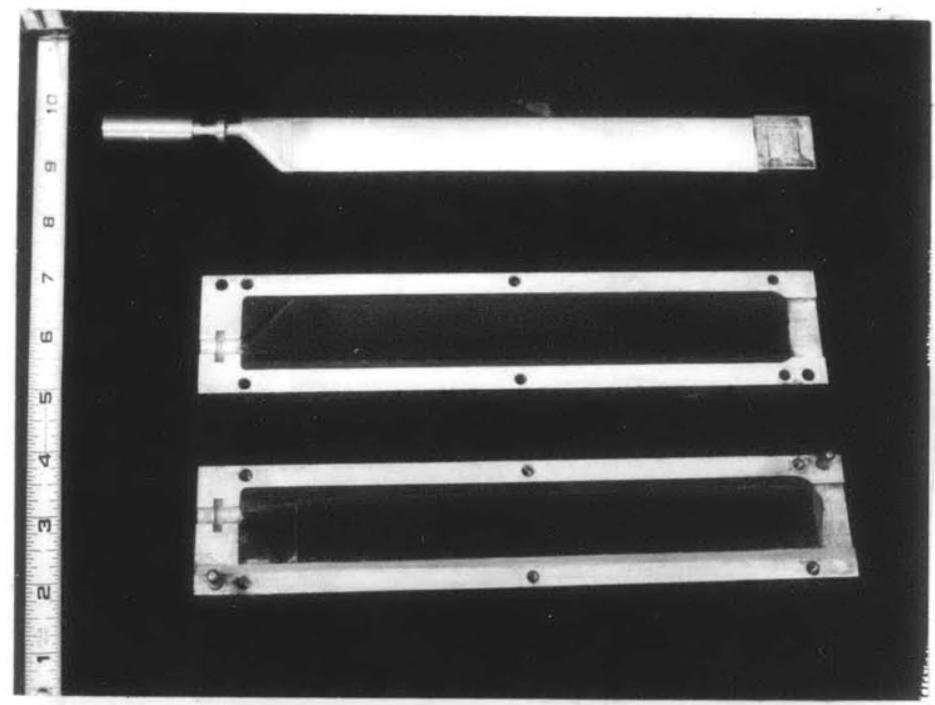

Fig. 40a. Aluminum model and epoxy mold.

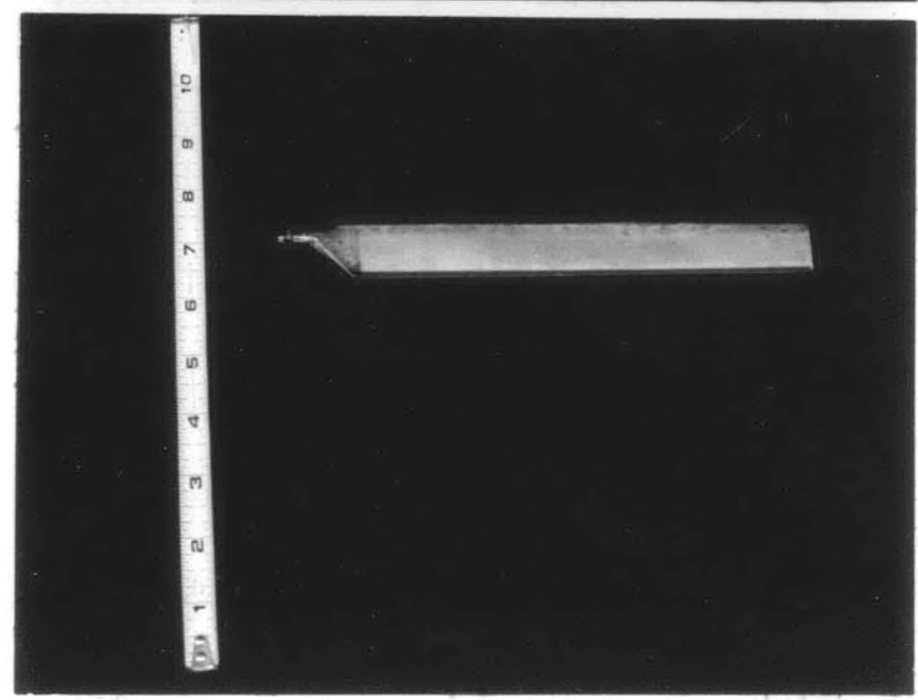

Fig. 40b. Finished blade.

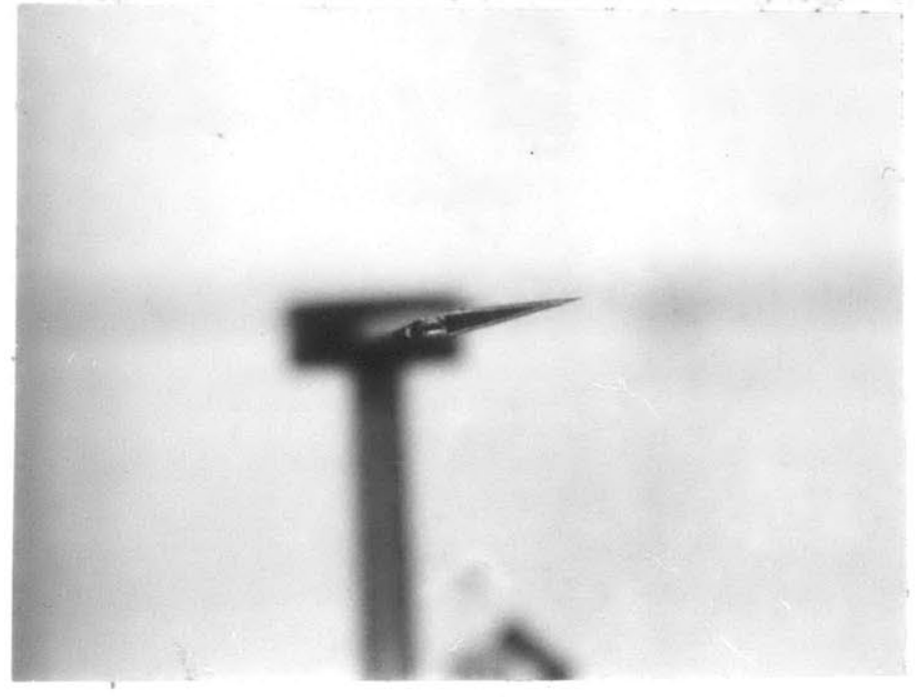

Fig. 40c. End view of finished blade. 


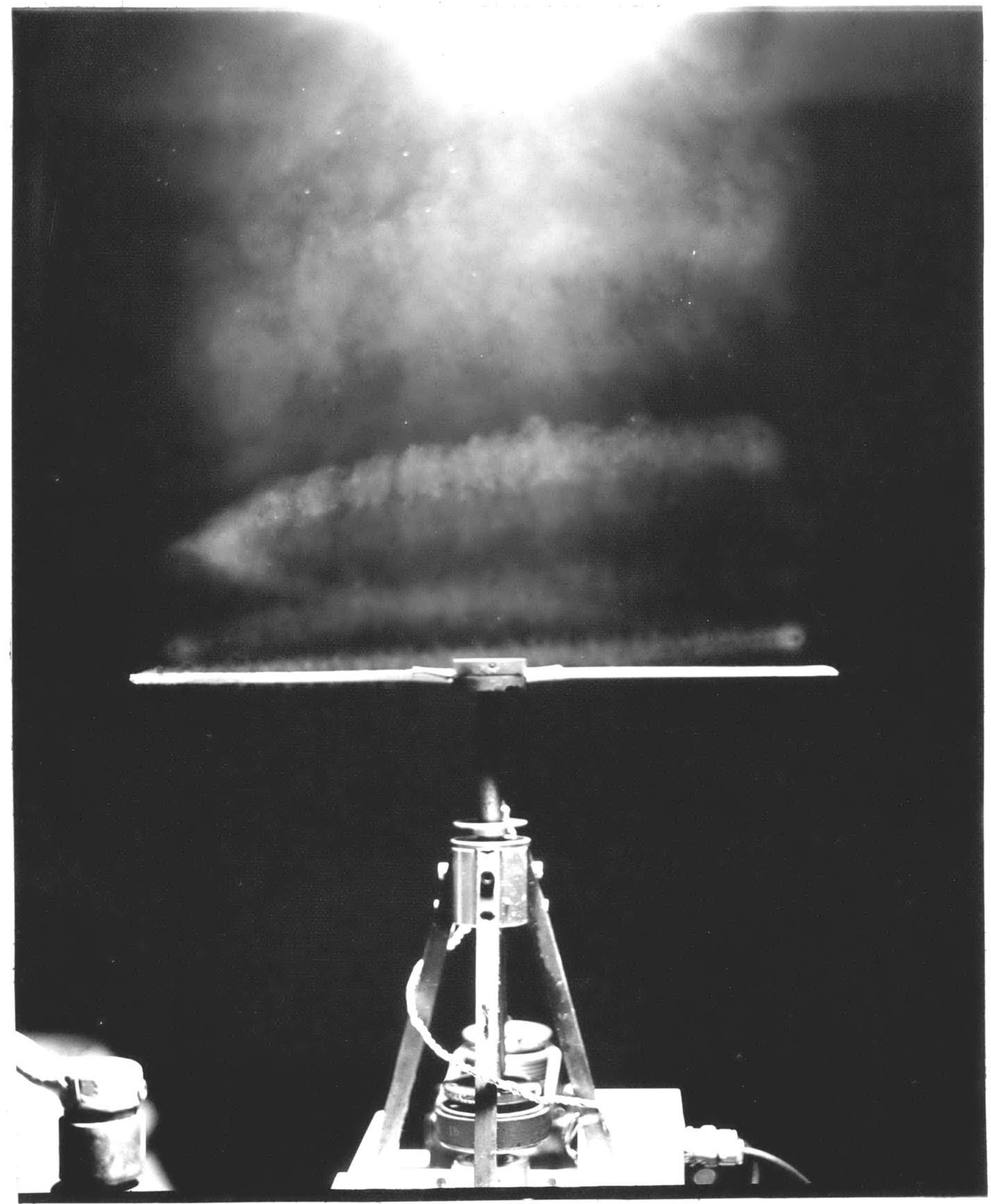

Fig. 41. Hovering rotor at 3200 rpm with smoke ejected at rotor tips. 


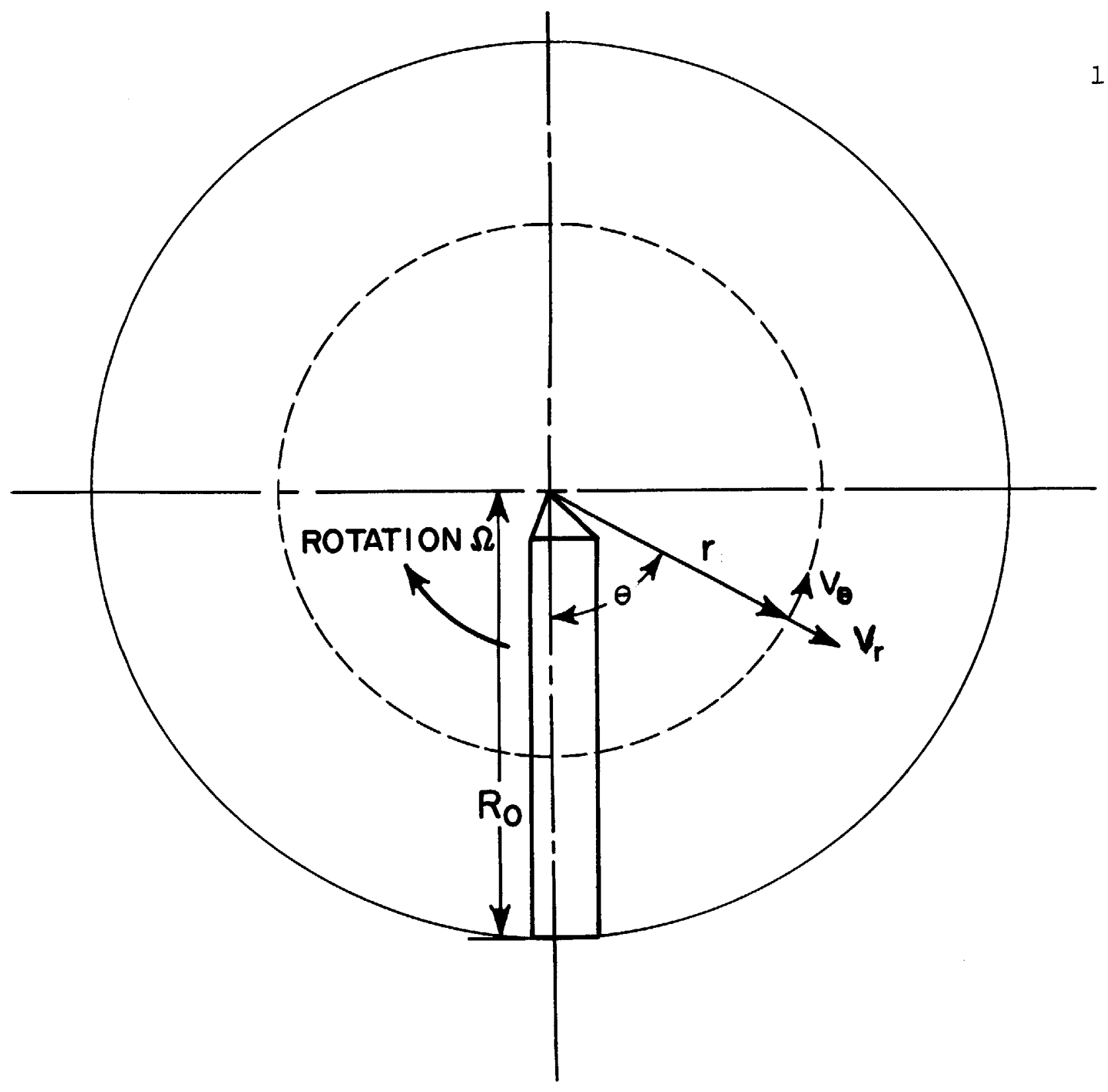

115.

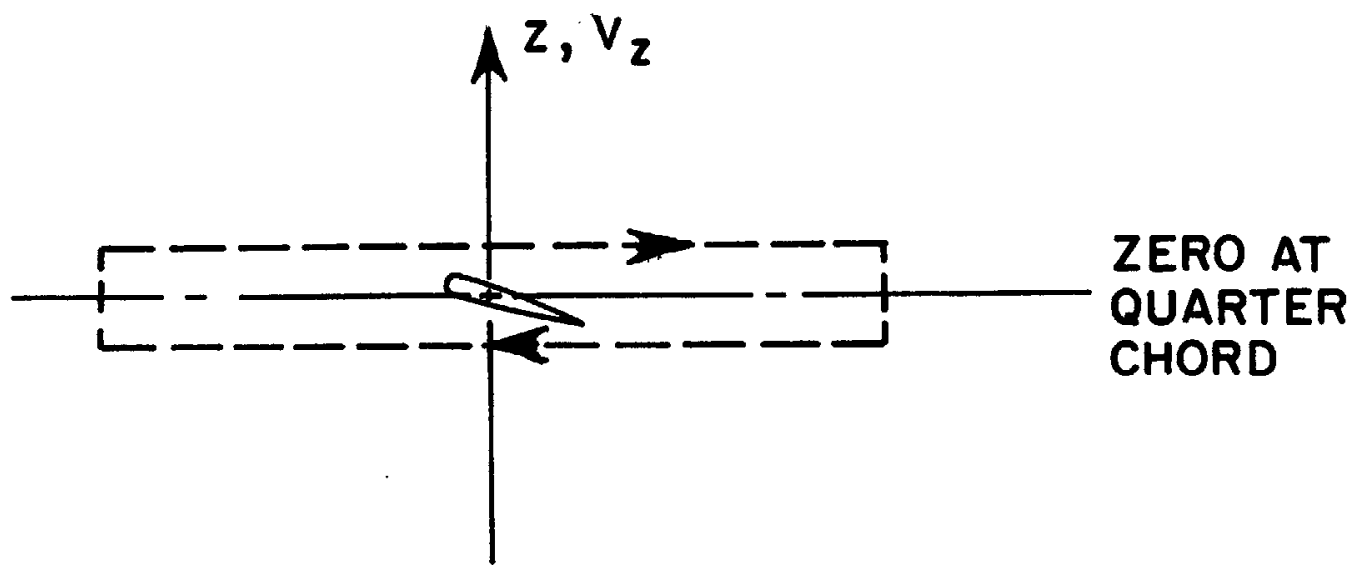

Fig. 42 Coordinate system 


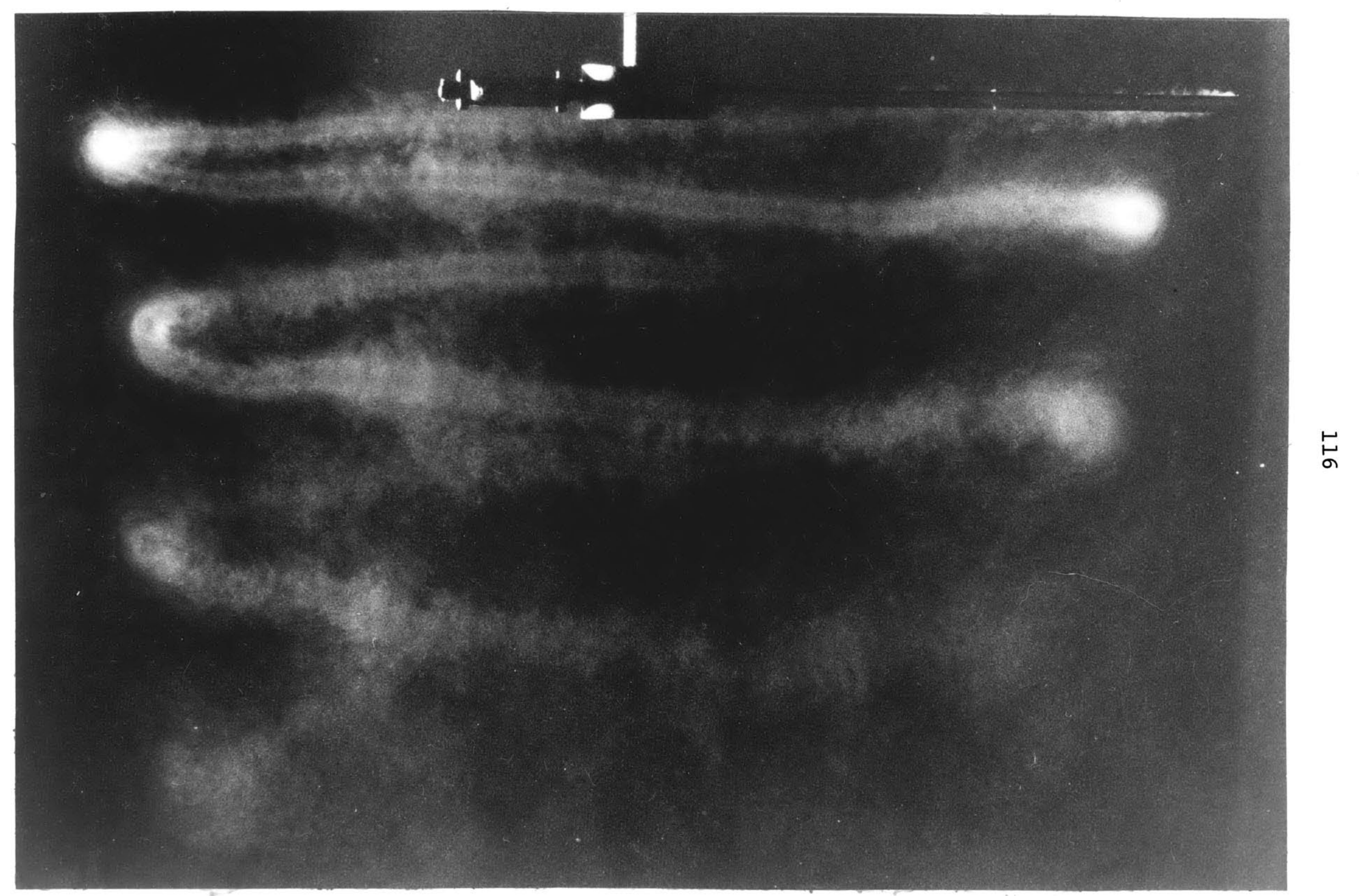

Fig. 43. Tip vortex showing wave structure 


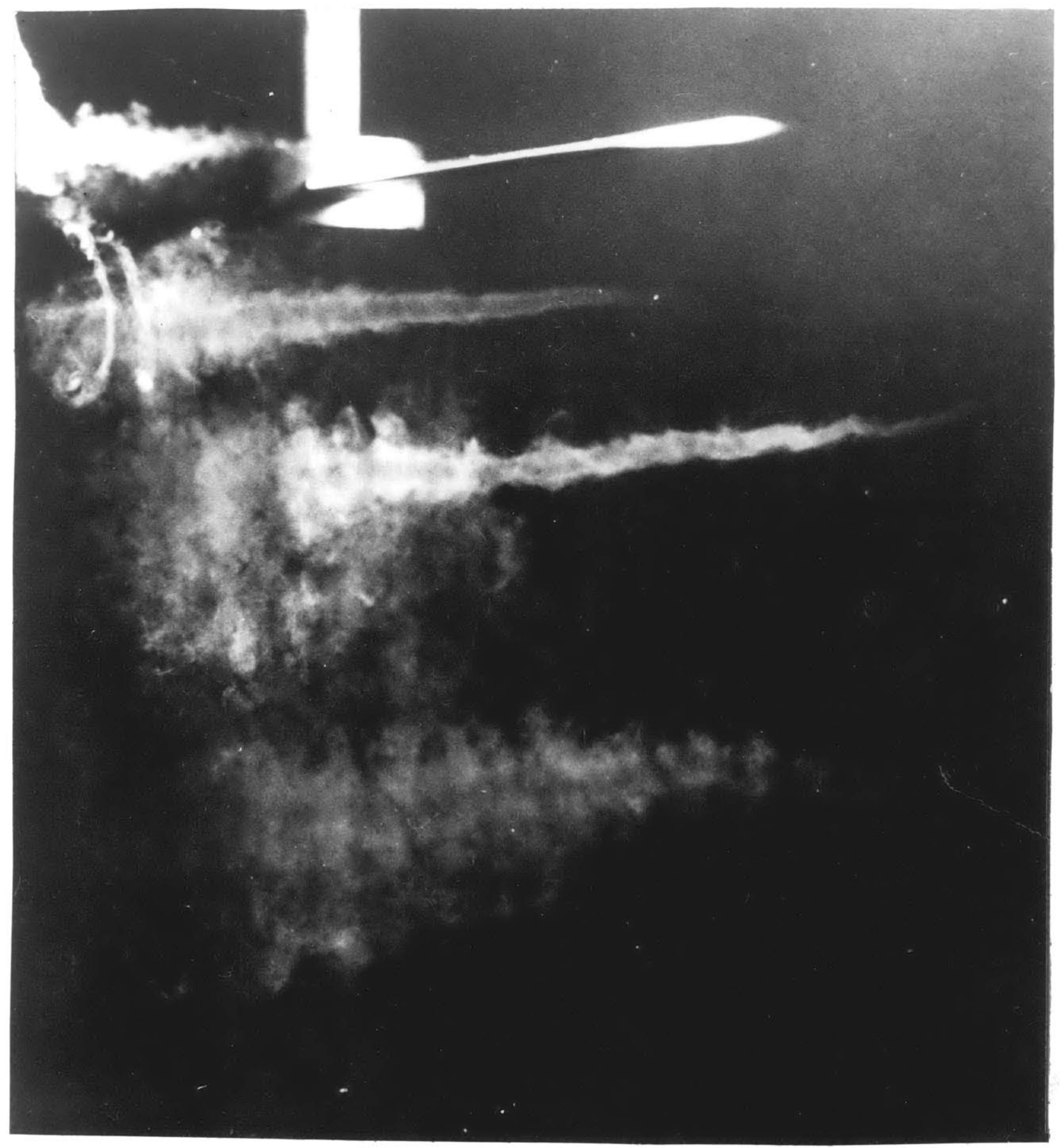

Fig. 44. Strobe photograph showing flow up the tip vortex towards the blade. 
118

Fig. 45

Strobe Photograph with Smoke

Released from Slit on Top of Blade

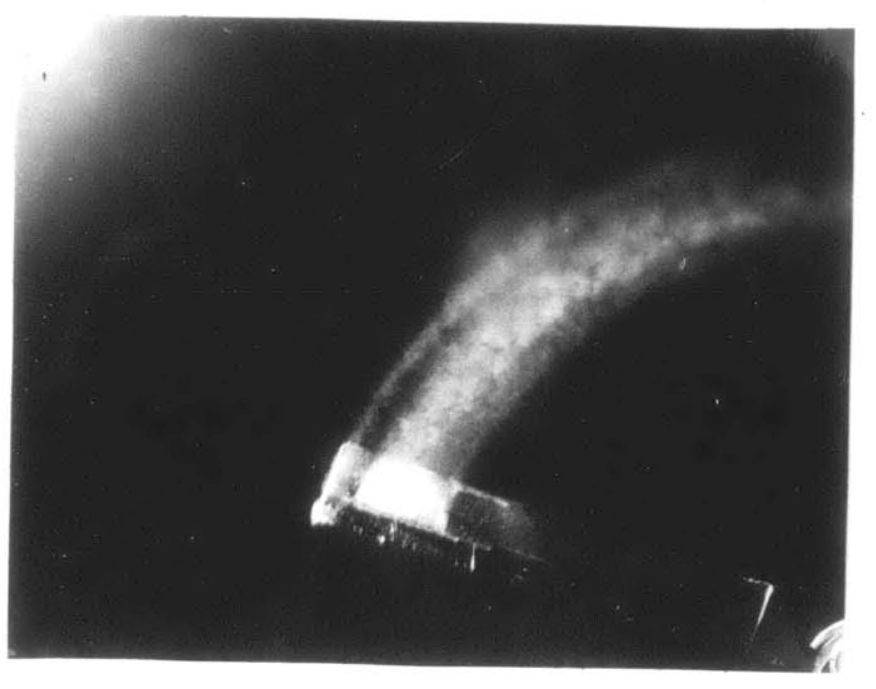




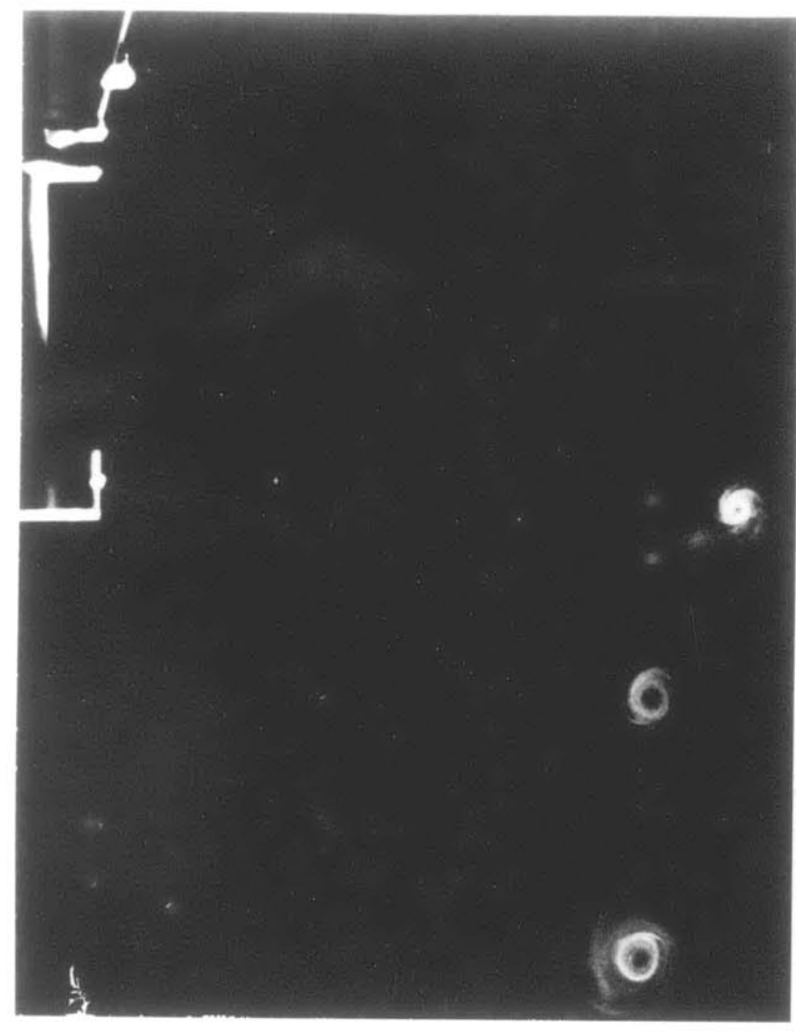

$\theta=45^{\circ}$

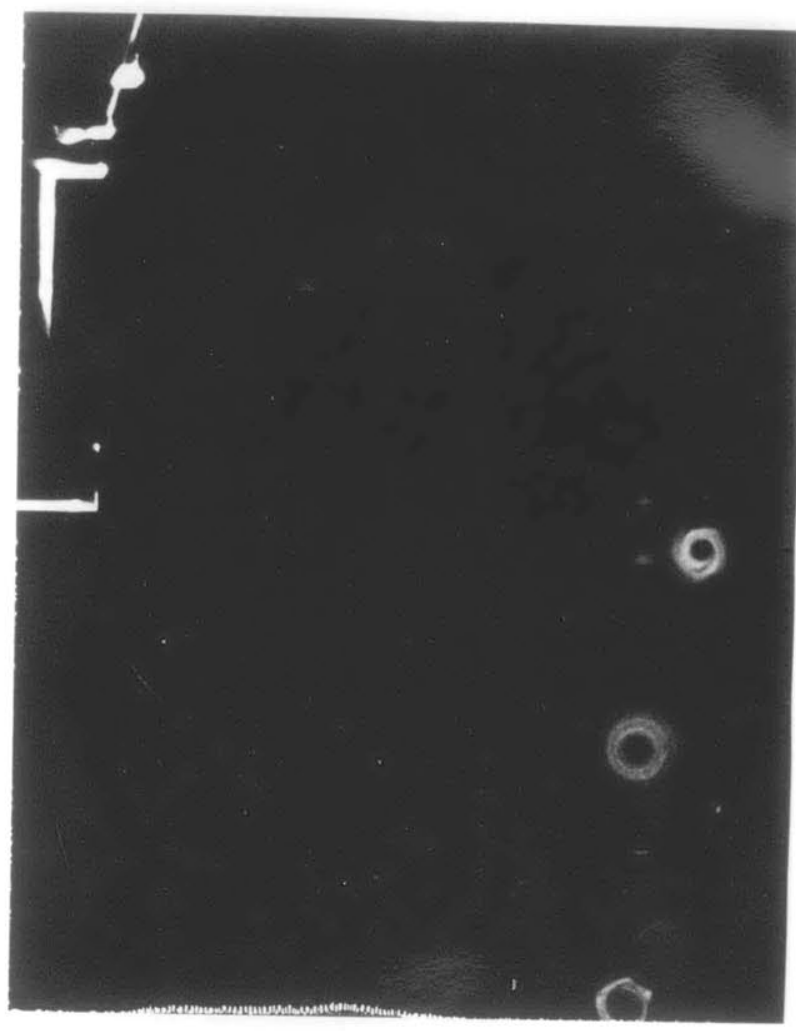

卢

Fig. 46. Samples of Photographs Used for Determining Vortex Core Position 


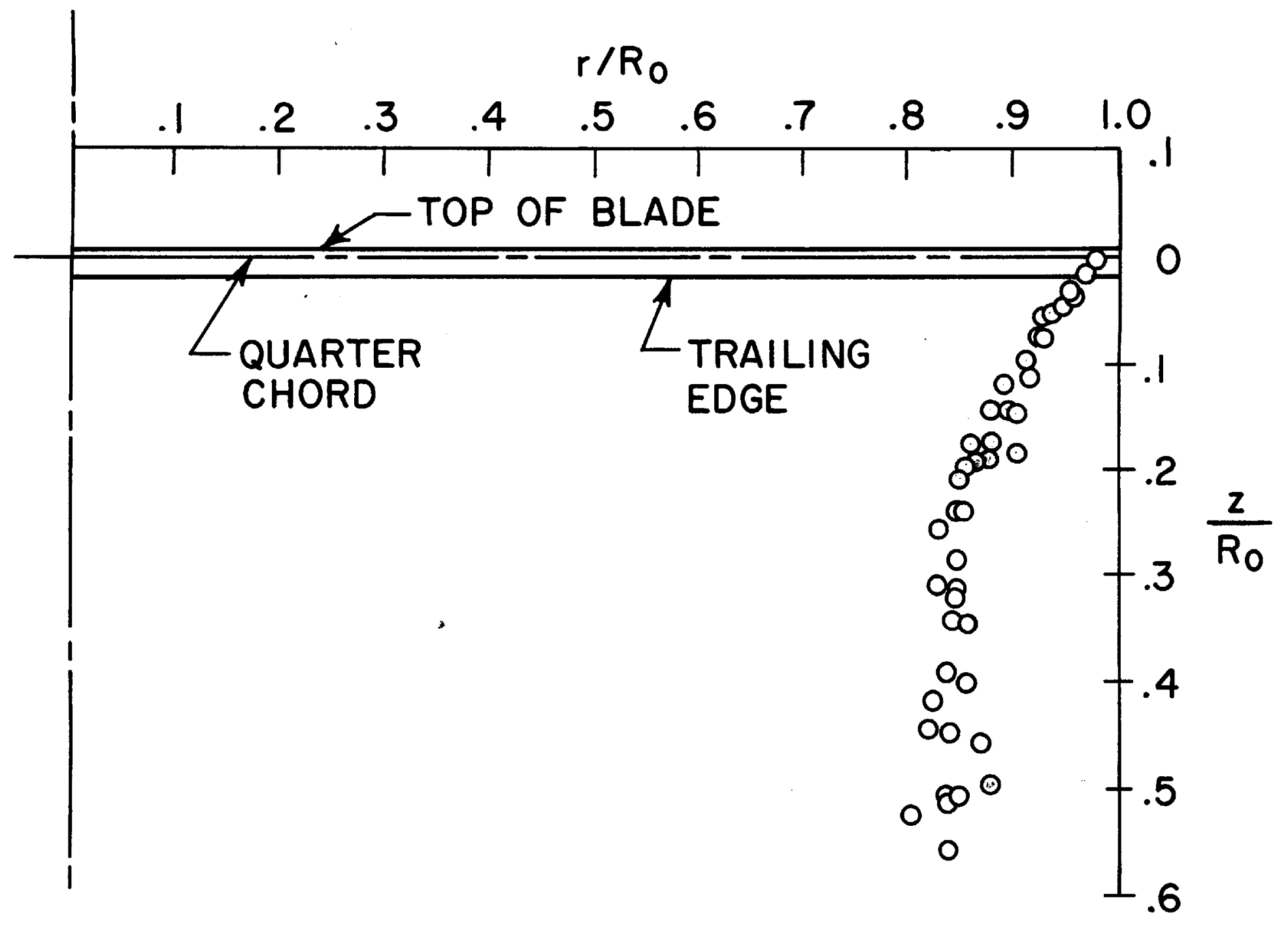




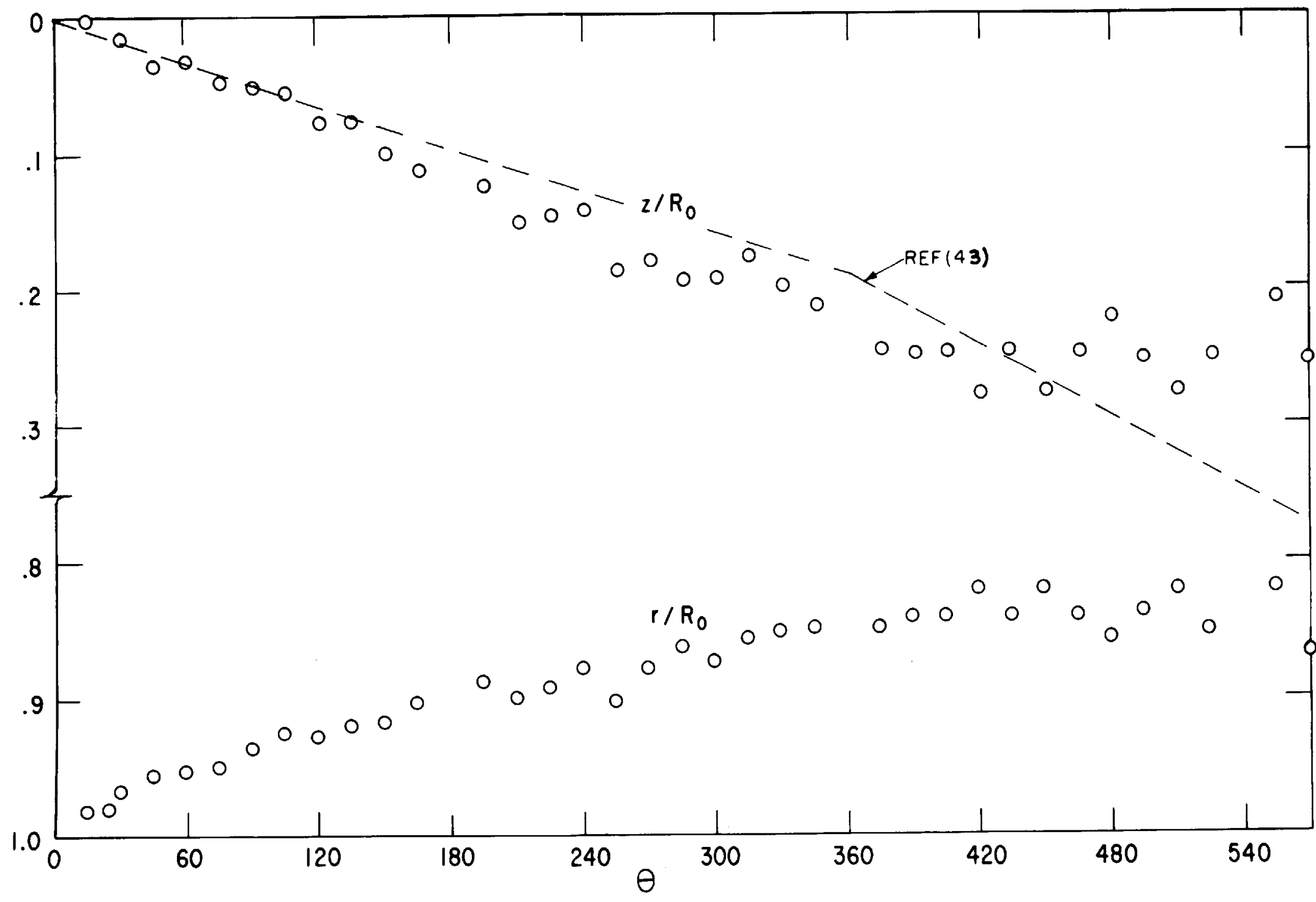

Fig. 48. Raciial and axial position of tip vortex. 


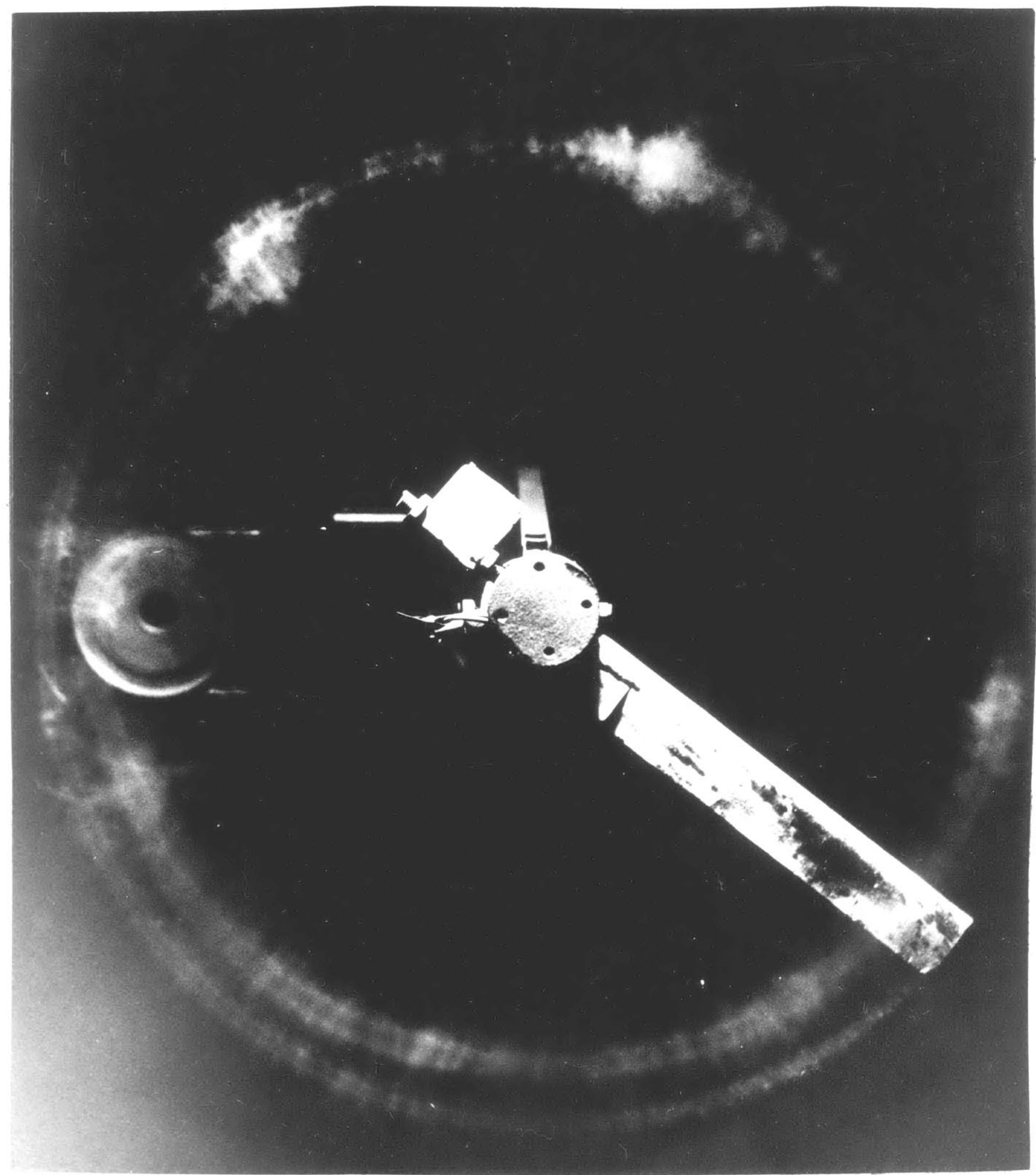

Fig. 49. Strobe photograph showing a gradual increase in core diameter and vortex breakdown. 


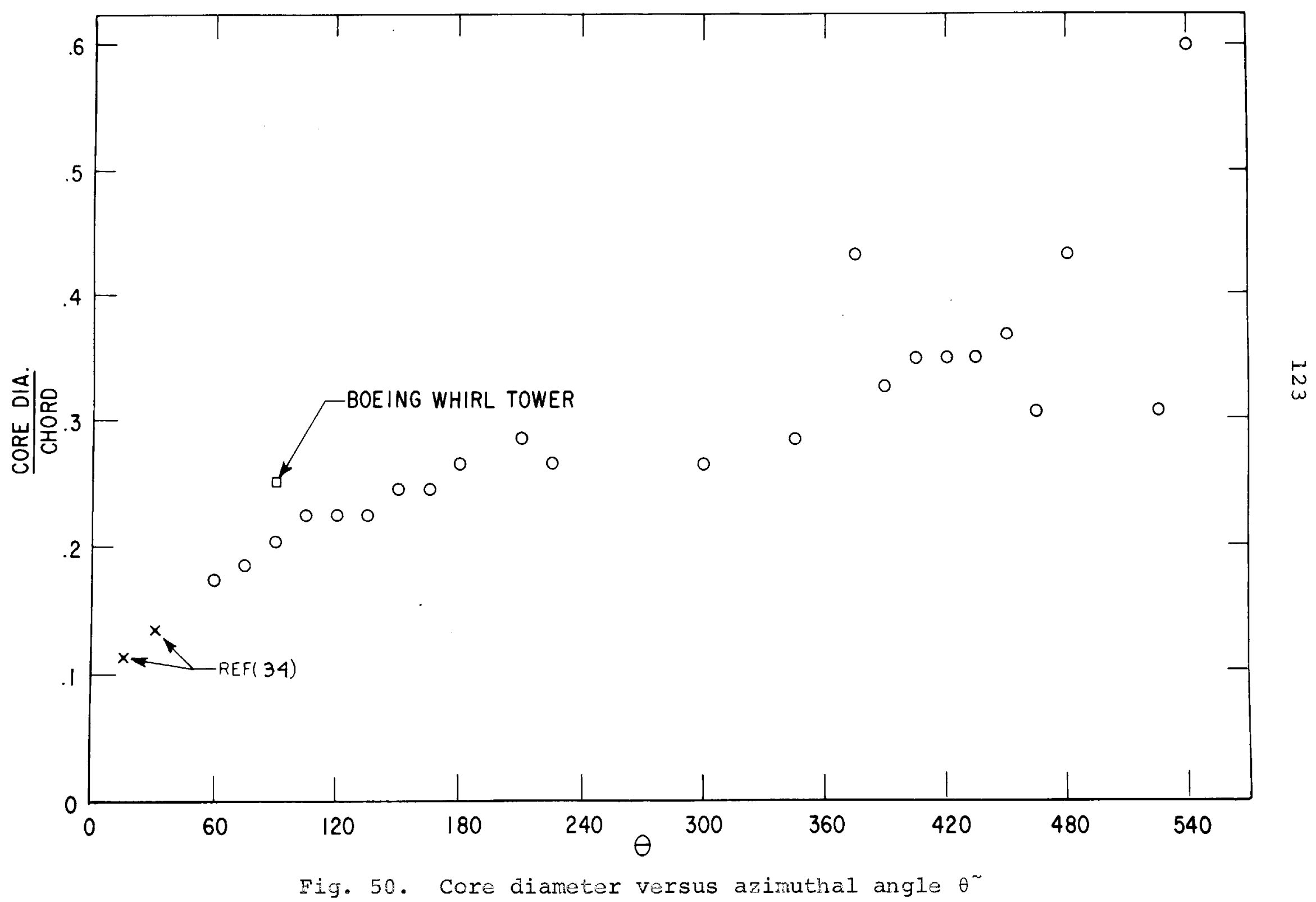




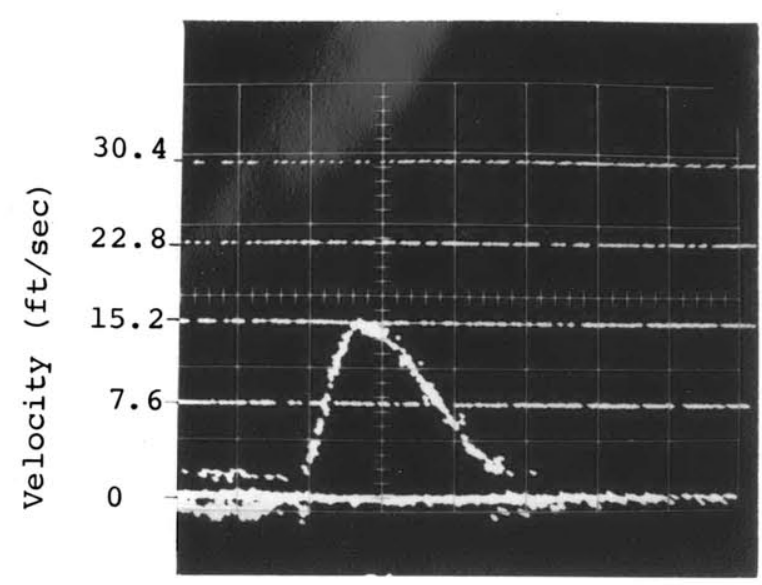

Fig. 5la $v_{\theta}$ component

$$
\begin{aligned}
& \mathrm{r} / \mathrm{r}_{0}=.555 \\
& \mathrm{z} / \mathrm{r}_{0}=+.0278
\end{aligned}
$$

Time scale $.5^{\mathrm{ms} / \mathrm{cm}}$

Velocity Direction-Positive

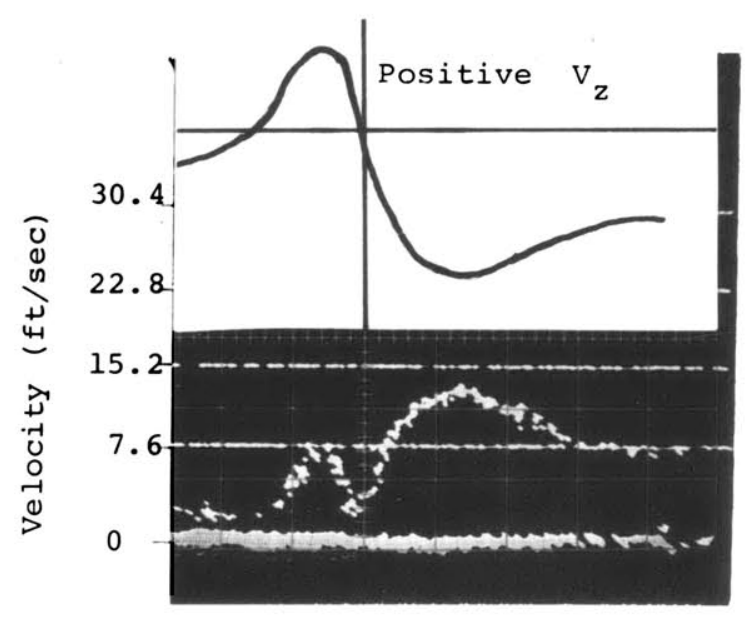

$\begin{aligned} \text { Fig. } 51 \mathrm{c} & v_{z} \text { component } \\ \mathrm{r} / \mathrm{r}_{0} & =.555 \\ \mathrm{z} / \mathrm{r}_{0} & =+.0278\end{aligned}$

Time Scale $.5^{\mathrm{ms} / \mathrm{cm}}$

Velocity Direction-Shown

in Insert

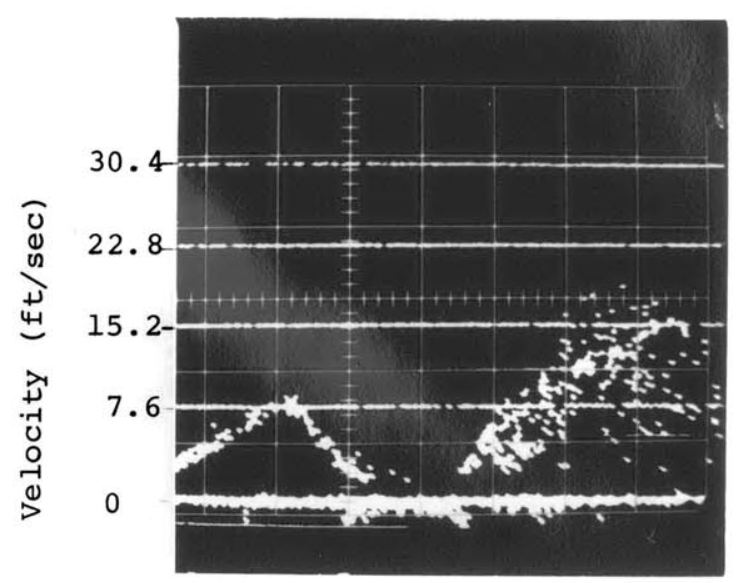

Fig. 51b $v_{\theta}$ component

$\mathrm{r} / \mathrm{r}_{\mathrm{O}}=.555$
$\mathrm{z} / \mathrm{r}_{\mathrm{O}}=-.0278$

Time Scale $.5^{\mathrm{ms} / \mathrm{cm}}$

Velocity Direction-Negative

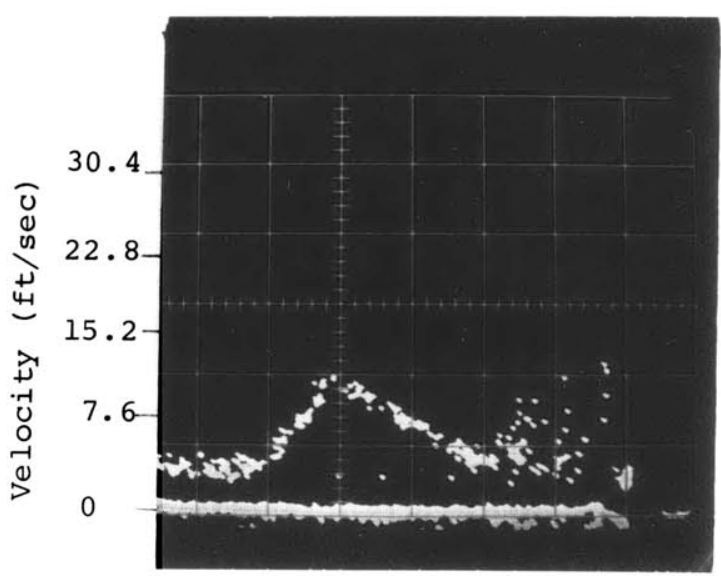

$\begin{aligned} \text { Fig. } 51 \mathrm{~d} & v_{z} \text { component } \\ \mathrm{r} / \mathrm{r}_{\mathrm{O}} & =.555 \\ \mathrm{z} / \mathrm{r}_{\mathrm{O}} & =-.0278\end{aligned}$

Time scale $.5^{\mathrm{ms} / \mathrm{cm}}$

Velocity Direction-Negative 

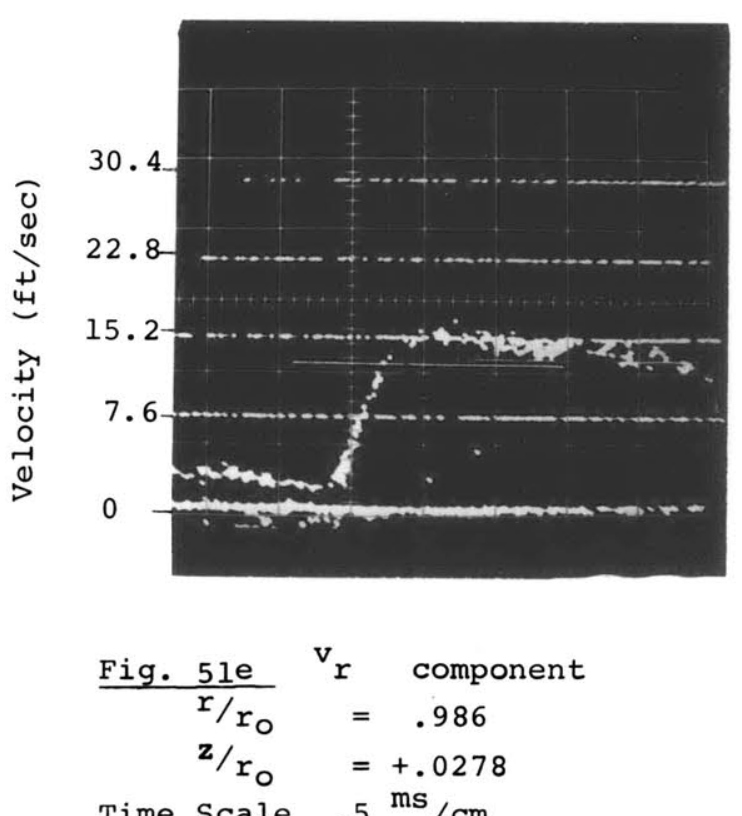

Velocity Direction-Negative
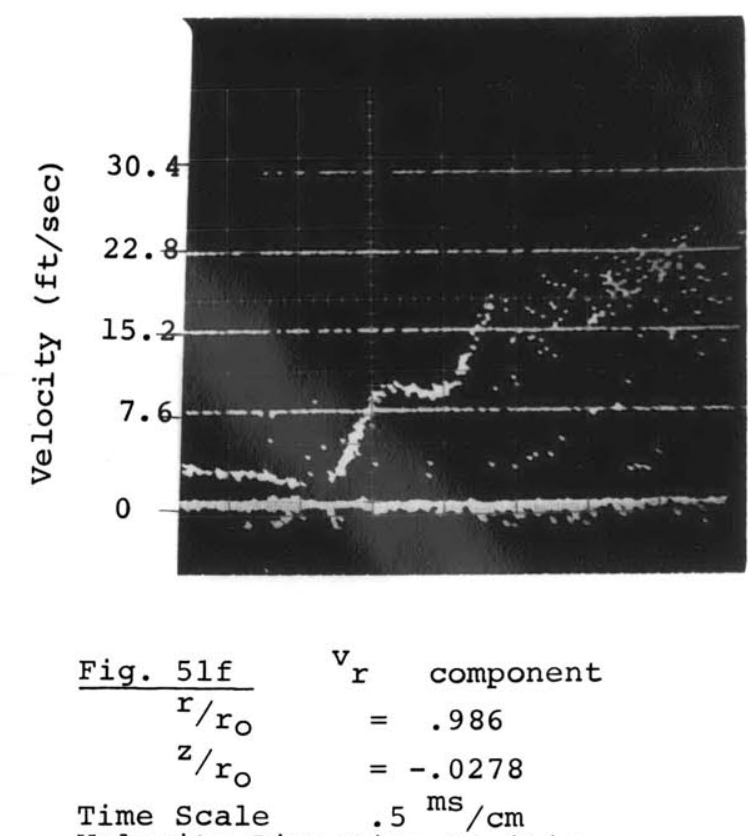

Velocity Direction-Positive
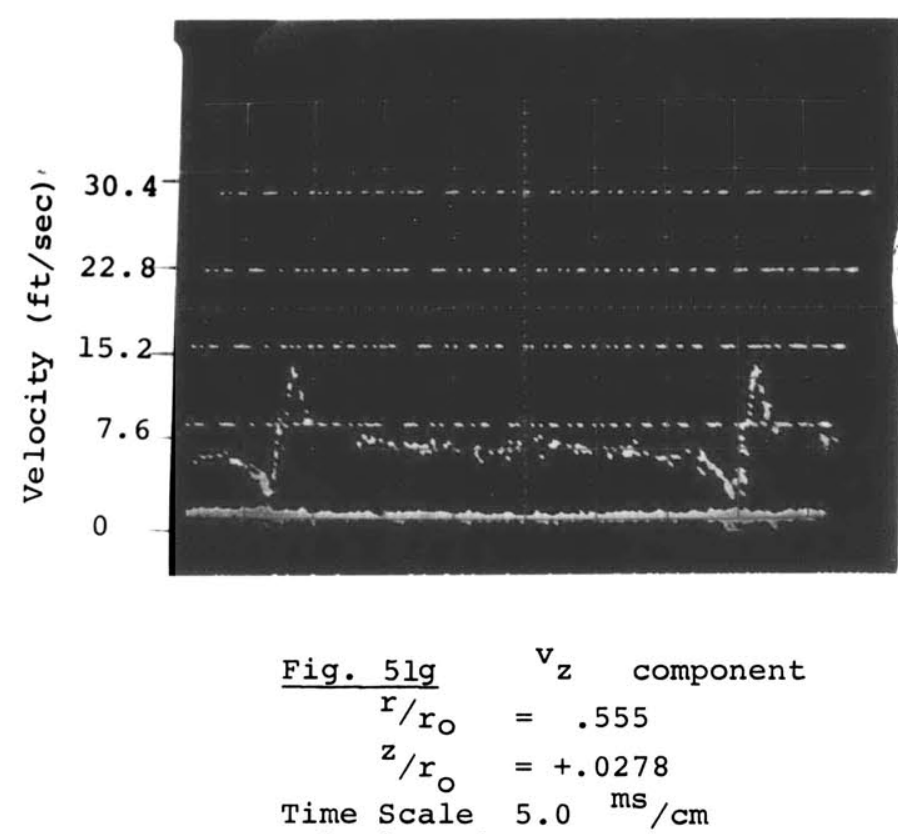

Velocity Direction-Negative 


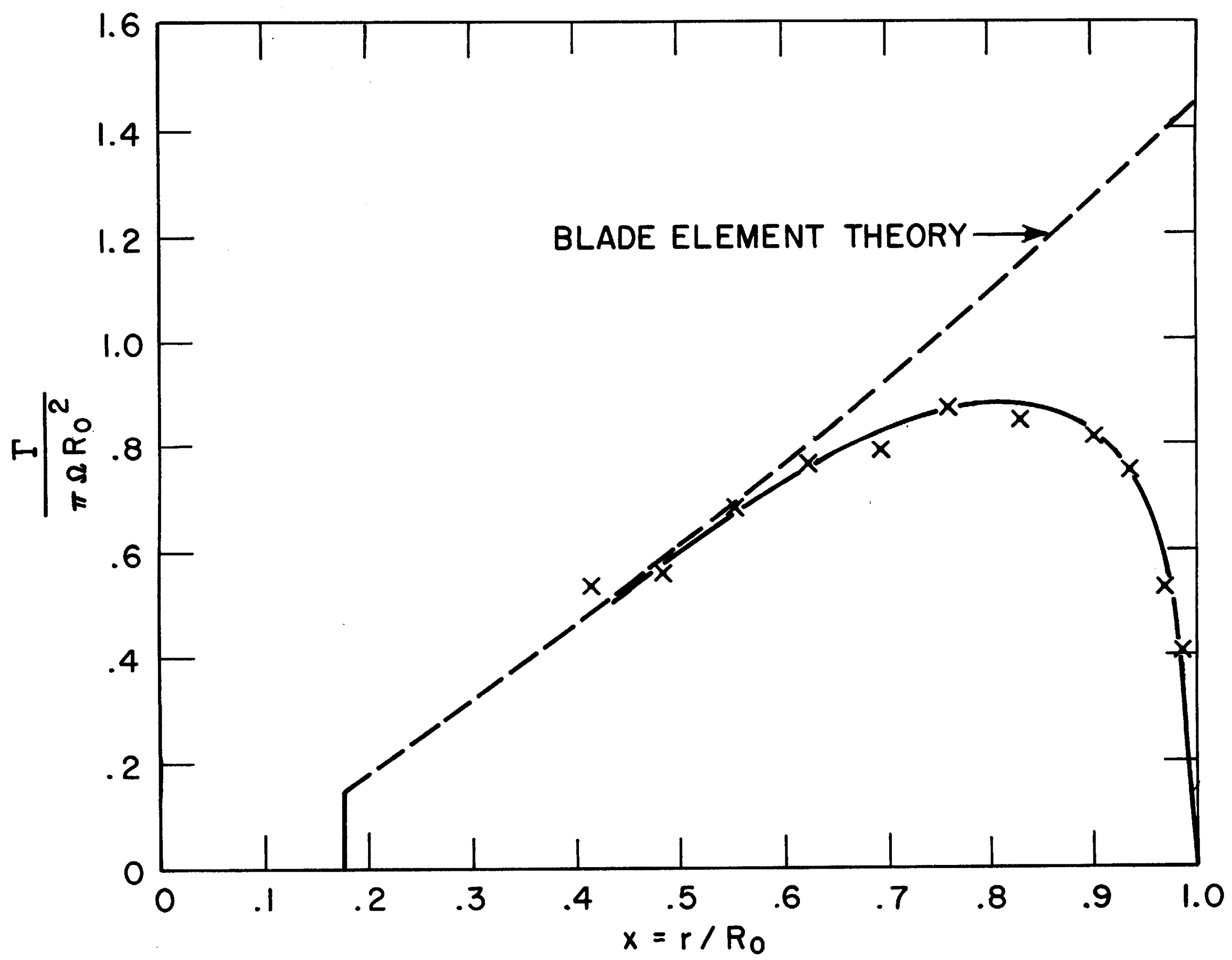




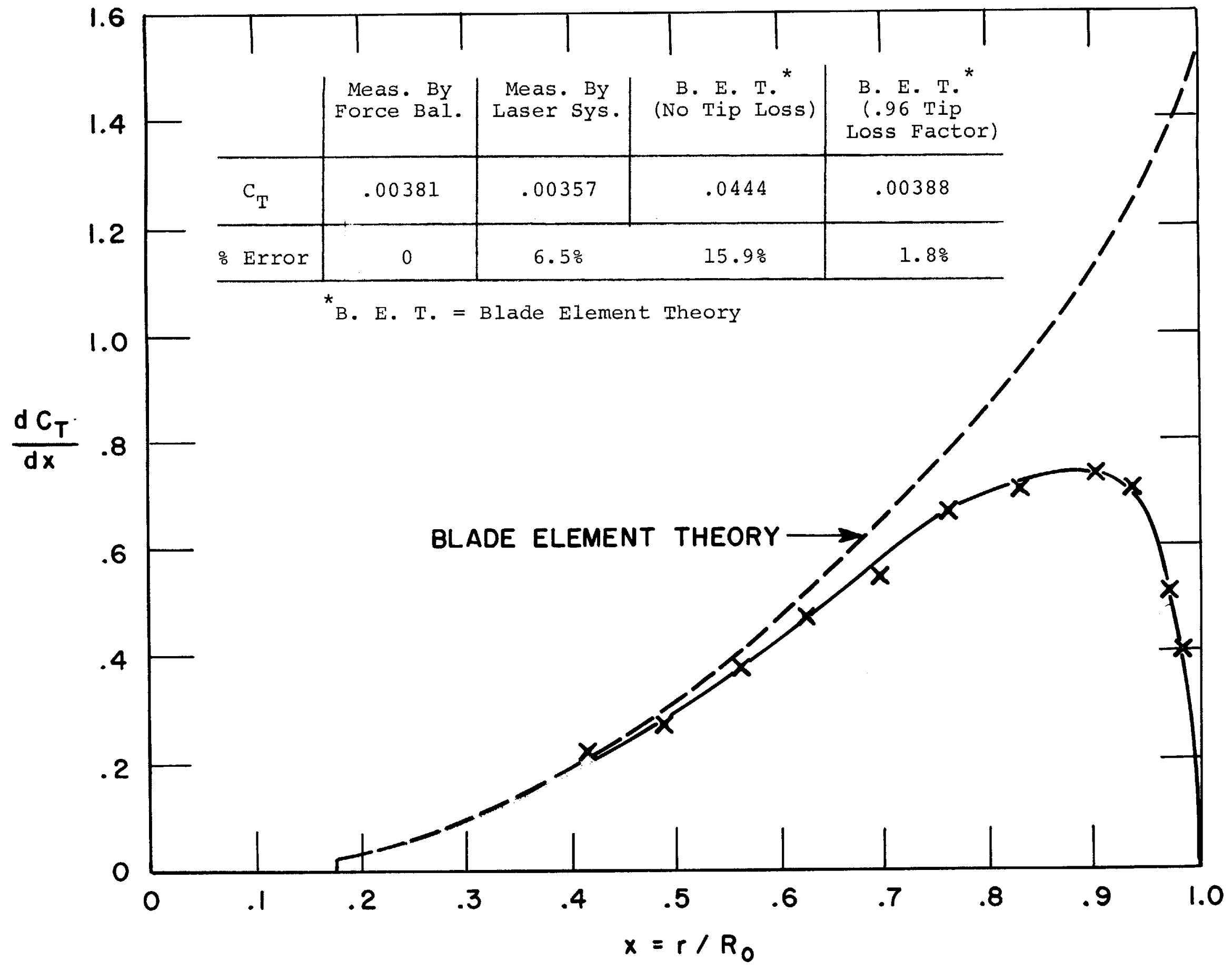

Fig. 53. Nondimensional Load Distribution Along Blade 


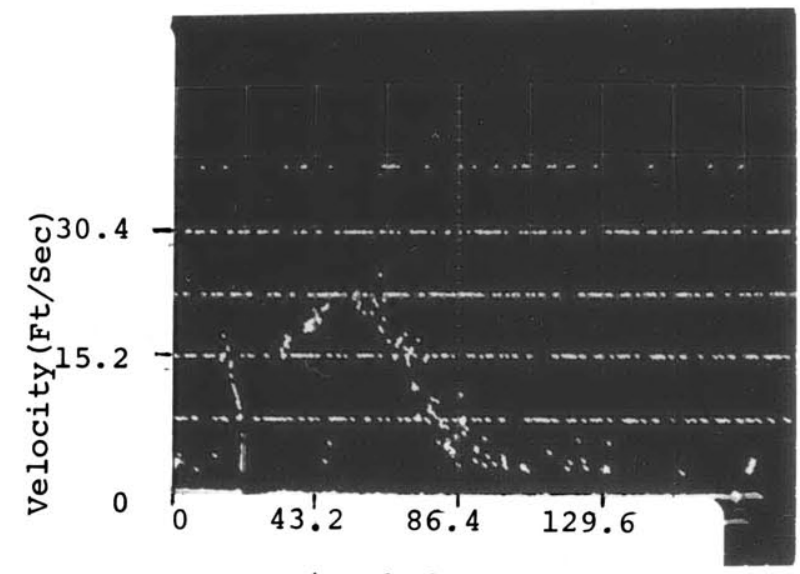

Azimuthal Angle

$\mathrm{v}_{\mathrm{z}}$ component

$\mathrm{r} / \mathrm{R}_{\mathrm{o}}=.937 \mathrm{z} / \mathrm{R}_{0}=-.0146$

Velocity direction-negative Time Scale $=2 \mathrm{mS} / \mathrm{cm}$

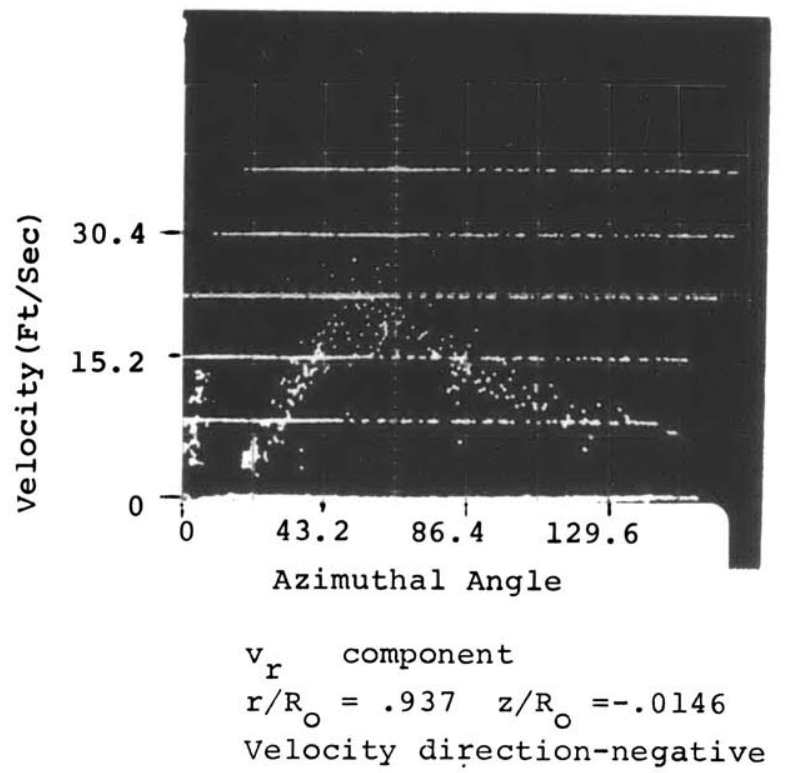

Fig. 54. Examples of LDV Lata near the tip vortex 


\section{BIOGRAPHY}

John Sullivan was born in Elkhart, Indiana on January 27, 1945. He attended grammar school and high school in Painesville, Ohio before beginning college at General Motors Institute in Flint, Michigan. After two years he left school and spent a year designing lubrication machinery. He returned to college at the University of Rochester, Rochester, New York where he received an S. B. degree in June 1967. He began studying at MIT in september 1967 receiving the M.S. degree June 1969. The title of his M. S. thesis is "Design of a Variable Phase Velocity Traveling Wave Pump." 